\title{
Further Generalization of Generalized Verma Modules
}

\author{
By
}

\section{Akihiko GYoJA*}

\section{$\S 0$. Introduction}

0.1. Let $G$ be a complex semisimple Lie group, $B$ a Borel subgroup, $P$ a parabolic subgroup containing $B, \mathfrak{g}=\operatorname{Lie}(G), \mathfrak{b}=\operatorname{Lie}(B), \mathfrak{p}=\operatorname{Lie}(P)$, and $L$ a finite dimensional irreducible $U(\mathfrak{p})$-module, where $U(-)$ denotes the enveloping algebra. A $U(\mathrm{~g})$-module of the form $U(\mathrm{~g}) \otimes_{U(\mathfrak{p})} E$ is called a generalized Verma module [24] and, in the special case where $\mathfrak{p}=\mathfrak{b}$, it is called a Verma module (cf. [9] and its references).

In the course of proving the Kazhdan-Lusztig conjecture [21], it was shown $[1]$, [8] that the Verma modules correspond to the local cohomologies at the $B$-orbits on $G / B$ via the localization functor. Thus it is natural to ask what are the $U(\mathrm{~g})$-modules corresponding to the local cohomologies at the $B$-orbits on $G / P$.

In this paper, we shall give an answer to this problem. It turns out that here appears a further generalization of the generalized Verma modules. We shall construct these $U(\mathrm{~g})$-modules in a purely algebraic way as follows. Let $\mathfrak{p}^{2}$ be the set of linear characters of the Lie algebra $\mathfrak{p}, A$ the ring of polynomial functions on $\mathfrak{p}^{2}$, and $c: \mathfrak{p} \rightarrow A$ the canonical homomorphism, which we shall consider as an $A$-valued character of a Cartan subalgebra, say $\mathrm{t}$, contained in $\mathfrak{b}=\operatorname{Lie}(B)$. Let $\lambda$ be the lowest weight of a finite dimensional irreducible $\mathfrak{p}$ module, $W$ the Weyl group, $W_{I}$ the Weyl subgroup of $W$ corresponding to $P$, and $w$ an element of $W$ which is longest in the coset $w W_{I}$. Let $U_{A}(-)=U(-)$ $\otimes_{C} A$ and define the 'universal' Verma module $M_{A}(w(c+\lambda-\rho)-\rho)$ by $M_{A}(w(c+$ $\lambda-\rho)-\rho)=U_{A}(\mathfrak{g}) \otimes_{U_{A}(\mathfrak{b}), w(c+\lambda-\rho)-\rho} A$, where $\rho$ is the half of the sum of the positive roots. Note that $w(c+\lambda-\rho)-\rho$ is not fully universal as a character of $t$ but it is universal among the characters lying on a certain facet with respect to the reflection group $W$ (translated by $w(\lambda-\rho)-\rho)$. Hence $M_{A}(w(c+$ $\lambda-\rho)-\rho$ ) resembles to reducible Verma modules and we can construct its quotient $V_{A}(w, c+\lambda, \mathfrak{p})$ in the same way as the construction of the simple quotient

Communicated by M. Kashiwara, December 4, 1991.

1991 Mathematics Subject Classification : 17B10

* Department of Fundamental Sciences, Faculty of Integrated Human Studies, Kyoto University, Kyoto 606-01, Japan. 
of the usual Verma module. The $U(\mathrm{~g})$-module investigated in this paper is the specialization of $V_{A}(w, c+\lambda, \mathfrak{p})$

$$
V(w, \lambda, \mathfrak{p})=V_{A}(w, c+\lambda, \mathfrak{p}) \otimes_{A} C,
$$

where $C$ is considered as a trivial $A$-module. (In other words, $V(w, \lambda, \mathfrak{p})$ is obtained from $V_{A}(w, c+\lambda, \mathfrak{p})$ by the specialization $c \rightarrow 0$.)

In contrast with the case of the usual generalized Verma modules, the most difficult point in the study of our $\mathrm{g}$-modules is the character formula, which will be proved in (6.3). Once we get the character formula, we can deduce several consequences from it. For instance, we show in (6.8) that our g-modules are a generalization of the generalized Verma modules, and, we construct in $\$ 7$ a resolution of our $\mathrm{g}$-module by Verma modules which is a generalization of the resolution of a finite dimensional representation constructed by BernsteinGelfand-Gelfand [2].

0.2. Using the character formula, we can also show that our $U(\mathrm{~g})$-module $V(w, \lambda, \mathfrak{p})$ actually corresponds to the local cohomology at a $B$-orbit of $G / P$. See (6.6). This fact enables us a $\mathscr{D}$-module theoretic study of our $U(\mathrm{~g})$-modules, by which we get an irreducibility criterion (9.13) for generalized Verma modules in terms of the $b$-functions of the semi-invariants. (See (9.2) for the semiinvariants.) Our irreducibility criterion is far different from, and unfortunately, less complete than the Jantzen's one [16], for we need to assume the antidominancy in order to use the generalities concerning the localization functor [1]. In this regard, see (9.14).

0.3. Let us explain our motivation. Assume that $g$ is simple, the nilpotent radical $\mathfrak{u}$ of $\mathfrak{p}$ is commutative, and a Levi subalgebra $\mathfrak{l}$ of $\mathfrak{p}$ is normalized by the longest element of the Weyl group. Let $L$ be the Levi subgroup of $P$ corresponding to $\mathfrak{l}$. Then it is known that ( $L$, adjoint action, $\mathfrak{l}$ ) is an irreducible regular prehomogeneous vector space, that there is an irreducible polynomial $f$ on $\mathfrak{u}$ which is relatively $L$-invariant, and that there is a unique fundamental weight $\approx$ which can be extended to a character of the Lie algebra p. (See [30], [12] for prehomogeneous vector spaces, and [25], [27] for there special kind of prehomogeneous vector spaces.) Let $b(s)$ be the Bernstein-Sato polynomial (= $b$-function) of $f$. In [31], S. Suga observed a relation between the simplicity of the generalized Verma module $M(\lambda)=U(\mathfrak{g}) \otimes_{U(\mathfrak{p}), \lambda \sigma} \boldsymbol{C}$ and the zeros of $b(s)$. (More precisely, $M(\lambda)$ is simple if and only if $b(\lambda-j) \neq 0$ for $j=$ $1,2, \cdots$.) The original motivation of the present work was to explain and generalize this observation.

Roughly speaking

$$
\text { ( } L \text {, adjoint action, } \mathfrak{l}) \risingdotseq(L \text {, left action, } G / P) \text {. }
$$


At one hand, we have the $\mathscr{D}$-module $\mathscr{D} f^{\lambda}$, which is related to the left hand side of (0.3.1). We can show that $\mathscr{D} f^{\lambda}$ is simple if and only if $b(\lambda-j) \neq 0$ for any $j \in Z$. On the other hand, we can expect that we get a $\mathscr{D}$-module, say $\mathscr{M}(\lambda)$, on $G / P$ by "localizing" the generalized Verma module $M(\lambda)$ as in [1]. Then $\mathscr{M}(\lambda)$, which is related to the right hand side of $(0.3 .1)$, would be simple if and only if $M(\lambda)$ is simple. Hence, by showing that $\mathscr{M}(\lambda) \div \mathscr{D} f^{\lambda}$, we would be able to explain the observation of Suga to some extent.

In this paper, we have tried to realize this idea and get (9.13), which is our first result in this direction, although it is still unsatisfactory.

0.4. A deeply related problem is studied by M. Kashiwara [20]. The relation between the present work and [20] will become clear in [13].

0.5. This paper consists of 9 sections. In Section 1, we define a new generalization $V(w, \lambda, \mathfrak{p}$ ) of Verma modules in (1.3) (cf. (4.1.1)) and give the basic lemma (1.12), which is used to prove the character formula. In Sections 2 and 3 , we review some known facts about the twisted $\mathscr{D}$-modules and the localization functor, respectively. In Section 4, we construct a certain $\mathrm{g}$-module, which is used to deduce the character formula from (1.12). In Section 5, we prove Proposition 5.2, which is used in (9.4). In Section 6, we prove the character formula in (6.3). Using it, we prove in (6.6) that the dual g-module of $V(w, \lambda, \mathfrak{p})$ corresponds to the local cohomology at $B w P / P$. In (6.7)-(6.9), we study the relation between our $g$-modules $V(w, \lambda, \mathfrak{p})$ and the usual generalized Verma modules. In Section 7 , we construct a resolution of $V(w, \lambda, \mathfrak{p})$ by the Verma modules, which is a generalization of the resolution of a finite dimensional representation constructed by Bernstein-Gelfand-Gelfand. In Section 8, we give a simplicity criterion (8.4) for a certain type of $\mathscr{D}$-modules, which is used in Section 9 to obtain an irreducibility criterion (9.13) for the generalized Verma modules.

0.6. The author would like to express his thanks to M. Kashiwara, Hisayosi Matsumoto and K. Nishiyama for their comments.

Convention. We denote the complex (resp. rational) number field by $\boldsymbol{C}$ (resp. $\boldsymbol{Q}$ ), the rational integer ring by $\boldsymbol{Z}$, and we put $\boldsymbol{N}=\{0,1,2, \cdots\}$. If two objects, say $X$ and $Y$, are naturally isomorphic, we often write $X=Y$.

\section{§1. A Generalization of Verma Modules}

1.0. In this section, we define a new generalization of Verma modules, and prove a basic lemma, which will be used to prove the character formula (6.3). First we review basic facts concerning Lie algebras in order to fix notations. We define our generalization of Verma modules in (1.3). After 
studying elementary properties of our modules, we give a basic lemma in (1.12). The remainder of the section is devoted to the proof of this lemma.

1.1. Let $G$ be a connected reductive group over the complex number field $C, B$ a Borel subgroup of $G, T$ a maximal torus contained in $B, W=N_{G}(T) / T$, and $g, \mathfrak{b}$ and $\mathfrak{t}$ the Lie algebras of $G, B$ and $T$, respectively. Let $\mathrm{t}^{2}=\operatorname{Hom}_{C}(\mathrm{t}, C)$, $R\left(\subset \mathrm{t}^{2}\right)$ be the root system of $(\mathrm{g}, \mathrm{t}), \mathrm{g}(\alpha)$ the root subspace of $\mathrm{g}$ corresponding to $\alpha \in R, R_{+}$the set of $\alpha \in R$ such that $\mathrm{g}(\alpha) \subset \mathfrak{b}, R_{-}=-R_{+}$, and $\mathfrak{n}_{ \pm}=\sum_{\alpha \in R_{ \pm}} \mathfrak{g}(\alpha)$. Let $\Pi=\left\{\alpha_{1}, \cdots, \alpha_{l}\right\}$ be the simple roots, $\Pi^{\vee}=\left\{\alpha_{1}^{\llcorner}, \cdots, \alpha_{l}^{\check{ }}\right\}$ the simple coroots, $\left\{\widetilde{\sigma}_{1}, \cdots, \widetilde{\varpi}_{l}\right\}$ the fundamental weights, and $\left\{\widetilde{\varpi}_{1}^{r}, \cdots, \widetilde{\varpi}_{l}^{\sim}\right\}$ the fundamental coweights. For $\alpha \in R$, let $\alpha^{2} \equiv \mathrm{t}$ be the corresponding coroot. Let $Q=\sum_{i=1}^{l} Z \alpha_{2}$ and $Q_{+}=\sum_{i=1}^{l} N \alpha_{i}$. Define $\mu \leqq \lambda\left(\mu, \lambda \in \mathrm{t}^{2}\right)$ if $\lambda-\mu \in Q_{+}$. Let $r_{\alpha}$ be the reflection with respect to $\alpha$, and $S=\left\{r_{\alpha} \mid \alpha \in \Pi\right\}$. For $w \in W$, denote its length by $l(w)$. Let $\leqq$ be the Bruhat order in $W$, where the identity element is minimal.

Let $I$ be a subset of $S, W_{I}$ the subgroup of $W$ generated by $I, w_{I}$ the longest element of $W_{I}, \Pi_{I}=\left\{\alpha \equiv I I \mid r_{\alpha} \in I\right\}, R_{I}$ the root subsystem of $R$ generated by $\Pi_{I}, \mathfrak{l}=\mathfrak{l}(I)=\mathfrak{t}+\sum_{\alpha \in R_{I}} \mathrm{~g}(\alpha), \mathfrak{l}_{ \pm}=\mathfrak{l}_{ \pm}(I)=\sum_{\alpha \in R_{ \pm} \backslash R_{I}} \mathrm{~g}(\alpha), \mathfrak{p}=\mathfrak{p}(I)=\mathfrak{l}+\mathfrak{l}_{+}$, and $\mathfrak{p}_{-}=\mathfrak{p}_{-}(I)=\mathfrak{l}+\mathfrak{l}_{-}$. We denote the connected subgroups of $G$ corresponding to $\mathfrak{l}, \mathfrak{n}_{ \pm}, \mathfrak{p}$ and $\mathfrak{p}_{-}$by $L=L(I), U_{ \pm}=U_{ \pm}(I), P=P(I)$ and $P_{-}=P(I)$, respectively. For $J, K \subset S$, let $\left(W_{J} \backslash W / W_{K}\right)_{s}$ (resp. $\left.\left(W_{J} \backslash W / W_{K}\right)_{l}\right)$ be the shortest (resp. longest) representatives of the double cosets in $W_{J} \backslash W / W_{K}$. For a subset $K$ of $S$, let $K^{\prime}=w_{S} K w_{S}(\subset S)$. Then $\left(W_{J} \backslash W / W_{K}\right)_{l}=\left\{w w_{S} \mid w \boxminus\left(W_{J} \backslash W / W_{K^{\prime}}\right)_{s}\right\}$. We write $\left(W / W_{I}\right)_{l}$ etc. for $\left(W_{\phi} \backslash W / W_{I}\right)_{l}$ etc.

Take a $\boldsymbol{Q}$-subspace $z_{\boldsymbol{Q}}$ of the center $z_{\text {such }}$ that $z_{\boldsymbol{Q}} \otimes \boldsymbol{C}=z_{\text {. }}$ Let $g_{\boldsymbol{Q}}$ be the $\boldsymbol{Q}$-linear span of a Chevalley basis of $[\mathrm{g}, \mathrm{g}]$ and $z_{Q}$. Put $\mathrm{t}_{\boldsymbol{Q}}=z_{\boldsymbol{Q}}+\sum_{\alpha \in R} Q \alpha^{2}$ and $\mathrm{t}_{I, Q}=\mathrm{t}_{\boldsymbol{Q}} / \sum_{\alpha \in \Pi_{I}} \boldsymbol{Q} \alpha^{2}$. Let $\mathrm{t}_{\boldsymbol{Q}}^{2}$ and $\mathrm{t}_{I, Q}^{2}$ be their dual spaces. For a $\boldsymbol{Q}$-algebra $A$, put $g_{A}=g_{Q} \otimes A$ etc. If $A=C$, we omit the suffix $A(=C)$. We identify $\mathfrak{1}_{I, A}$ with the $W_{I}$-invariant elements in $\operatorname{Hom}_{Q}\left(\mathrm{t}_{\boldsymbol{Q}}, A\right)=\operatorname{Hom}_{\boldsymbol{A}}\left(\mathrm{t}_{A}, A\right)$. We say that $\lambda \in \mathrm{t}^{2}$ is anti-dominant (resp. regular) if $\left\langle\lambda, \alpha^{2}\right\rangle \notin \boldsymbol{N} \backslash\{0\}$ (resp. $\neq 0$ ) for any $\alpha \in$ $R_{+}$. Let $\mathrm{t}_{a d}^{2}$ (resp. $\mathrm{t}_{r a d}^{2}$ ) be the set of $\lambda \in \mathrm{t}^{2}$ such that $\lambda-\rho$ is anti-dominant

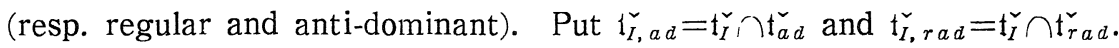

Let $k$ be a field of characteristic zero. For a Lie algebra $\mathfrak{a}$ over $k$, let $U(\mathfrak{a})=U_{k}(\mathfrak{a})$ be the enveloping algebra, and $Z(\mathfrak{a})$ the center of $U(\mathfrak{a})$. Express $z \subseteq Z(\mathrm{~g})$ as $z=\varphi(z)+z^{\prime}$ with $\varphi(z) \equiv U(\mathrm{t})$ and $z^{\prime} \equiv U(\mathrm{~g}) \mathfrak{n}_{+}$. Consider $\lambda \in \mathrm{t}^{2}$ as a character of $U(\mathrm{t})$. Let $\chi_{\lambda}=\lambda \circ \varphi$ and $U(\lambda, \mathrm{g})=U(\mathrm{~g}) / U(\mathrm{~g}) \operatorname{ker} \chi_{\lambda}$. Let $\rho=(1 / 2) \sum_{\alpha \in R_{+}} \alpha$ and $\gamma: U(\mathrm{t}) \rightarrow U(\mathrm{t})$ be the isomorphism defined by $\gamma(H)=H-\rho(H)$ for $H \equiv \mathrm{t}$. Then $\gamma \circ \varphi$ gives an isomporphism $Z(\mathrm{~g}) \rightarrow U(\mathrm{t})^{W V}$ (cf. $[9,7.4 .5]$ ), which is called the Harish-Chandra homomorphism. Here $U(\mathrm{t})^{W}$ denotes the totality of the $W$ invariant elements of $U(\mathrm{t})$. Note that $\lambda \circ \gamma \circ \varphi=\chi_{\lambda-\rho}$. Hence $\chi_{w \lambda-\rho}=\chi_{\lambda-\rho}$ and $U(w \lambda-\rho, g)=U(\lambda-\rho, g)$ for any $w \in W$.

For a $U_{k}(\mathrm{t})$-module $V$ and $\mu \in \mathrm{t}_{k}^{2}$, let $V_{\mu}$ be the set of $v \equiv V$ such that for any $H \in \mathrm{t}_{k}$, there exists an integer $n$ such that $(H-\mu(H))^{n} v=0$. We call $V_{\text {" }}$ 
the weight space of weight $\mu$. If $\operatorname{dim} V_{\mu}<\infty$ for any $\mu \in \mathrm{I}_{k}$, we define the character $\operatorname{ch}(V)$ of $V$ as the formal $\operatorname{sum} \operatorname{ch}(V)=\sum_{\mu \in t_{\breve{k}}}\left(\operatorname{dim} V_{\mu}\right) e^{\prime \prime}$.

Let $\tau$ be an automorphism of $g$ which normalizes $t$ and induces -1 on $t$. Then $\tau(\mathrm{g}(\alpha))=\mathrm{g}(-\alpha)$. For any $U(\mathrm{~g})$-module $M$, define a $\mathrm{g}$-module structure in $\operatorname{Hom}_{C}(M, C)$ by $\langle A f, u\rangle=-\langle f, \tau(A) u\rangle$ for $A € \mathrm{~g}, f \in \operatorname{Hom}_{C}(M, C)$ and $u=M$. If $M$ is a direct sum of weight spaces, and each weight space is of finite dimension, then put $M^{*}=\left\{f \equiv \operatorname{Hom}_{C}(M, C) \mid f\left(M_{\mu}\right)=0\right.$ except for finitely many $\left.\mu^{\prime}=\mathrm{t}^{\sim}\right\}=\oplus_{\mu \in t} \sim \operatorname{Hom}\left(M_{\mu}, \mathbb{C}\right)$. Then $\left(M^{*}\right)^{*}=M$, and $\operatorname{ch}\left(M^{*}\right)=\operatorname{ch}(M)$.

1.2. Verma module. Extend $\lambda \equiv \mathrm{t}_{k}^{-}=\operatorname{Hom}_{k}\left(\mathrm{t}_{k}, k\right)$ to a linear character of the Lie algebra $\mathfrak{b}_{k}$ by putting $\lambda \mid \mathfrak{i t}_{k} \equiv 0$. Let $k(\lambda)$ be the corresponding $U\left(\mathfrak{b}_{k}\right)$ module, $1_{\lambda}$ its basis element, and

$$
M(\lambda)=M_{k}(\lambda)=M_{k}\left(\lambda, \mathfrak{b}_{k}\right)=U\left(\mathfrak{g}_{k}\right) \otimes_{U\left(\mathfrak{b}_{k}\right)} k(\lambda),
$$

which is called a Verma module [33], [3] (cf. [9]). Denote its simple quotient by $V(\lambda)=V_{k}(\lambda)$.

1.3. A generalization of Verma modules. For a $k$-algebra 4 , put $U_{A}(\mathfrak{a})=$ $U_{k}(\mathfrak{a}) \bigotimes_{k} A$. For $a \equiv \mathrm{t}_{A}^{2}$, let $A(a)=U_{A}\left(\mathfrak{b}_{k}\right) /\left(U_{A}\left(\mathfrak{b}_{k}\right) \mathfrak{n}_{+, k}+\sum_{H \in t_{k}} U_{A}\left(\mathfrak{b}_{k}\right)(H-a(H))\right)$, $1_{a}$ be the element of $A(a)$ corresponding to $1 \in U_{A}\left(\mathfrak{b}_{k}\right)$, and $M_{A}(a)=U_{A}\left(\mathfrak{g}_{k}\right)$ $\otimes_{U_{A}\left(b_{k}\right)} \mathcal{l}(a)$, which we consider as a family of Verma modules. We can show that $M_{A}(a)$ is a free $U_{A}\left(\mathfrak{n}_{-, k}\right)$-module generated by $u(a)=u_{A}(a):=1 \otimes 1_{a}$. Let $M_{A}(a)_{+}=U_{A}\left(\mathfrak{n}_{-, k}\right) \mathfrak{n}_{-, k} u_{A}(a), J_{A}(a)$ be the (unique) maximal $U_{A}\left(\mathfrak{g}_{k}\right)$-submodule of $M_{A}(a)$ contained in $M_{A}(a)_{+}, V_{A}(a)=M_{A}(a) / J_{A}(a)$, and $v(a)=v_{A}(a):=\left(u_{A}(a) \bmod \right.$ $J_{A}(a)$ ).

Let $L$ be an affine subspace of $\mathfrak{t}_{k}^{2}, A=A(L)=A_{k}(L)$ the algebra $k[L]$ of polynomial functions on $L, c=c_{L}$ the natural homomorphism $\mathrm{t}_{k} \rightarrow A, M(L)=$ $M_{A}(L):=M_{A(L)}\left(c_{L}\right)=U_{A(L)}\left(\mathrm{g}_{k}\right) \otimes_{U_{A(L)}\left(\mathrm{b}_{k}\right)} A\left(c_{L}\right), M(L)_{+}=M_{A}(L)_{+}:=M_{A(L)}\left(c_{L}\right)_{+}, J(L)$ $=J_{A}(L):=J_{A(L)}\left(c_{L}\right)$, and $V(L)=V_{A}(L):=V_{A(L)}\left(c_{L}\right)$. Let $p$ be a prime ideal of $A(L), k(p)$ the residue field at $p$, and $M(L, p)=M(L) \otimes_{A(L)} k(p), V(L, p)=V(L)$ $\otimes_{A(L)} k(p)$ etc. If $p(\lambda)$ is the maximal ideal of $A(L)$ consisting of polynomial functions vanishing at $\lambda \subseteq L$, then $A(L) \rightarrow k(p(\lambda))=k$ is the evaluation at $\lambda$, which we shall denote by the same letter $\lambda$. Put

$$
V(L, \lambda)=V_{k}(L, \lambda):=V(L, p(\lambda))=V(L) \otimes_{A(L), \lambda} k .
$$

Let $p(\eta):=\{0\}, K=K(L):=k(p(\eta)), M_{K}(L):=M(L, p(\eta))=M(L) \otimes_{A(L)} K, V_{K}(L)$ $:=V(L, p(\eta))=V(L) \otimes_{A(L)} K$, etc. (specializations at the generic point $\eta$ of $L$ ). Note that $K(L)$ is the quotient field of $A(L)$. Thus we can consider the composition $\eta=\eta_{L}\left(\in \mathrm{t}_{K}\right)$ of $\mathrm{t}_{k} \stackrel{{ }^{c}}{\rightarrow} . A(L) \rightarrow K(L)$, the Verma module $M_{K}\left(\eta_{L}\right)$, and its simple quotient $V_{K}\left(\eta_{L}\right)$.

Lemma 1.4. (1) $M_{K}\left(\eta_{L}\right)=M_{K}(L)$. (2) $V_{K}\left(\eta_{L}\right)=V_{K}(L)$. 
Proof. (1) is trivial. Consider $M_{A}(L)$ as a submodule of $M_{K}(L)$. Since $V_{K}(\eta)=M_{K}(\eta) / J_{K}(\eta)$ and $V_{K}(L)=K \otimes\left(M_{A}(L) / J_{A}(L)\right)=M_{K}(\eta) / K J_{A}(L)$, it remains to prove that

$$
J_{K}(\eta)=K J_{A}(L) .
$$

Since $J_{K}(\eta) \cap M_{A}(L)$ is a $U_{A}\left(g_{k}\right)$-submodule of $M_{A}(L)$ contained in $M_{A}(L)_{+}$, we have $K J_{A}(L) \supset K\left(J_{K}(\eta) \cap M_{A}(L)\right)=J_{K}(\eta)$. On the other hand, $K J_{A}(L)$ is a $U_{K}\left(\mathfrak{g}_{k}\right)$-submodule of $M_{K}(\eta)$ contained in $M_{K}(\eta)_{+}$. Hence $K J_{A}(L) \subset J_{K}(\eta)$.

Lemma 1.5. (1) Let $\lambda \in L$. If $V_{k}(L, \lambda)$ is simple as a $U\left(\mathrm{~g}_{k}\right)$-module, then it is absolutely simple. (2) $V_{K}(L)$ is absolutely simple.

Proof. These assertions follow from (1.4, (2)), and [9, 2.6 .5 and 7.1.8, (iv)].

1.6. Field extension. Let $k^{\prime}$ be a field containing $k, L^{\prime}=L \otimes_{k} k^{\prime}, A^{\prime}=$ $A_{k^{\prime}}\left(L^{\prime}\right)$, and $K^{\prime}$ the quotient field of $A^{\prime}$. We can naturally consider $t_{k}$ (resp. $\left.\mathrm{t}_{k}^{r}\right)$ as a subspace of $\mathrm{t}_{k^{\prime}}\left(\right.$ resp. $\left.\mathfrak{t}_{k^{\prime}}^{\prime}\right)$. Then $\mathrm{t}_{k}^{\sim}=\left\{\lambda \equiv \operatorname{Hom}_{k^{\prime}}\left(\mathrm{t}_{k^{\prime}}, k^{\prime}\right) \mid \lambda\left(\mathrm{t}_{k}\right) \subset k\right\}$ and $L=\left\{\lambda:=L^{\prime} \mid \lambda\left(\mathrm{t}_{k}\right) \subset k\right\}$.

Lemma 1.7. (1) $V_{A}(L) \otimes_{A} A^{\prime}=V_{A^{\prime}}\left(L^{\prime}\right)$. (2) $V_{K}\left(\eta_{L}\right) \otimes_{K} K^{\prime}=V_{K}(L) \otimes_{K} K^{\prime}=$ $V_{K^{\prime}}\left(L^{\prime}\right)=V_{K^{\prime}}\left(\eta_{L^{\prime}}\right)$. (3) $V_{k}(L, \lambda) \otimes_{k} k^{\prime}=V_{k^{\prime}}\left(L^{\prime}, \lambda\right)$ for $\lambda \equiv L\left(\subset L^{\prime}\right)$.

Proof. (1) Since $M_{A}(L) \otimes_{A} A^{\prime}=M_{A^{\prime}}\left(L^{\prime}\right)$, it suffices to show that $A^{\prime} J_{A}(L)=$ $J_{A^{\prime}}\left(L^{\prime}\right)$. Since $M_{A^{\prime}}\left(L^{\prime}\right)=U_{A^{\prime}}\left(\mathfrak{n}_{-, k}\right) u_{A^{\prime}}(c) \cong U_{A^{\prime}}\left(\mathfrak{n}_{-, k}\right)$, wre can take a free $A^{\prime}$-basis $\left\{u_{1}, u_{2}, \cdots\right\}$ of $M_{A^{\prime}}\left(L^{\prime}\right)$ in $U_{k}\left(\mathfrak{n}_{-, k}\right) u_{A^{\prime}}(c)$. An element $u$ of $M_{A^{\prime}}\left(L^{\prime}\right)$ belongs to $J_{A^{\prime}}\left(L^{\prime}\right)$ if and only if $U\left(\mathrm{~g}_{k}\right) u \subset M_{A^{\prime}}\left(L^{\prime}\right)_{+}$. For $n \fallingdotseq \boldsymbol{N}$ and $u=\sum a_{i}^{\prime} u_{\imath} \subseteq \sum_{\imath \leqq n} A^{\prime} u_{\imath}$, this condition can be written as a system of homogeneous $A$-linear equations in $\left(a_{i}^{\prime}\right)_{\imath \leqq n} \equiv A^{\prime n}$. Since $A^{\prime}$ is flat over $A$, every solution $\left(a_{i}^{\prime}\right)$ in $A^{\prime n}$ can be expressed as an $A^{\prime}$-linear combination of solutions in $A^{n}[7$, Chap. $1, \S 2$, Corollary 2 of Proposition 13]. Hence $\left(\sum_{\imath \leqq n} A^{\prime} u_{\imath}\right)_{1} \backslash J_{A^{\prime}}\left(L^{\prime}\right)=A^{\prime} \cdot\left(\sum_{\imath \unlhd n} A u_{i} \cap J_{A}(L)\right)$. Letting $n \rightarrow \infty$, we get $J_{A^{\prime}}\left(L^{\prime}\right)=A^{\prime} J_{A}(L)$. (2) By $(1.4,(2))$, it suffices to prove the equality $V_{K}(L) \otimes K^{\prime}=V_{K^{\prime}}\left(L^{\prime}\right)$, which can be proved in the same way as above. (3) By (1), we have $V_{k^{\prime}}\left(L^{\prime}, \lambda\right)=V_{A^{\prime}}\left(L^{\prime}\right) \otimes_{A^{\prime}, \lambda} k^{\prime}=\left(V_{A}(L) \otimes_{A} A^{\prime}\right) \otimes_{A^{\prime}, \lambda} k^{\prime}=$ $V_{A}(L) \otimes_{A, \lambda} k^{\prime}=\left(V_{A}(L) \otimes_{A, \lambda} k\right) \otimes_{k} k^{\prime}=V_{k}(L, k) \otimes_{k} k^{\prime}$.

1.8. Let $r=\operatorname{dim} L$. If $0 \Subset L$, we can find a $k^{\prime}$-linear basis $\lambda_{1}, \cdots, \lambda_{r}$ of $L^{\prime}$ contained in $L$. In this case, put $\lambda_{0}=0$. If $0 \notin L$, we can find linearly independent elements $\lambda_{0}, \lambda_{1}, \cdots, \lambda_{r} \in L$ such that $L^{\prime}=\left\{\sum_{i=0}^{r} a_{\imath} \lambda_{\imath} \mid a_{i} \equiv k^{\prime}, \sum_{i=0}^{r} a_{\imath}\right.$ $=1\}$. This equality also holds in the case where $0 \equiv L$. Take an element $\lambda=$ $\sum_{i=0}^{r} a_{i} \lambda_{i}$ of $L^{\prime}$. Let $k\langle\lambda\rangle$ be the subfield of $K^{\prime}$ generated by $k$ and $\lambda\left(t_{k}\right)$. Then $k\langle\lambda\rangle=k\left(a_{1}, \cdots, a_{r}\right)$. Hence the transcendental degree tr. $\operatorname{deg}_{k} k\langle\lambda\rangle$ of $k\langle\lambda\rangle$ over $k$ is at most $r=\operatorname{dim} L$. 
1.9. If $k^{\prime}=\boldsymbol{C}$ and tr. $\operatorname{deg}_{\boldsymbol{Q}} k<\infty$, then $\left\{\lambda \in L^{\prime} \mid \operatorname{tr} . \operatorname{deg}_{k} k\langle\lambda\rangle=\operatorname{dim} L\right\} \neq \boldsymbol{\phi}$ is stable with respect to the translations by elements of $L$ and hence everywhere dense in $L^{\prime}\left(\subset \mathrm{t}_{C}^{2}=\mathrm{t}^{2}\right)$.

Lemma 1.10. If tr. $\operatorname{deg}_{k} k\langle\lambda\rangle=\operatorname{dim}_{k} L$, then $V_{k}\left(L^{\prime}, \lambda\right)$ is simple.

Proof. By $(1.5,(1))$ and $(1.7,(3))$, we may assume that $k^{\prime}=k\langle\lambda\rangle$. Let $I_{L}=$ $\left\{\varphi \leqq k\left[\mathrm{t}_{k}^{\sim}\right] ; \varphi \mid L \equiv 0\right\}, H_{1}, \cdots, H_{r}$ be elements of $\mathrm{t}_{k}$ such that $\lambda_{\imath}\left(H_{j}\right)=\delta_{i j}(0 \leqq i \leqq r$, $1 \leqq i \leqq r)$, and $\bar{H}_{2}$ the corresponding elements of $k\left[\mathrm{t}_{k}{ }^{2}\right] / I_{L}$. Then $A$ is the symmetric algebra $k\left[\bar{H}_{1}, \cdots, \bar{H}_{r}\right]$ and $K$ is its quotient field $k\left(\bar{H}_{1}, \cdots, \bar{H}_{r}\right)$. Since $a_{\imath}=\lambda\left(H_{\imath}\right)(1 \leqq i \leqq r)$ are algebraically independent over $k$, the homomorphism $\lambda$ : $4=k\left[\bar{H}_{1}, \cdots, \bar{H}_{r}\right] \rightarrow k\left[\lambda\left(H_{1}\right), \cdots, \lambda\left(H_{r}\right)\right]$ can be extended to an isomorphism $\lambda$ : $K=k\left(\bar{H}_{1}, \cdots, \bar{H}_{r}\right) \simeq k\left(\lambda\left(H_{1}\right), \cdots, \lambda\left(H_{r}\right)\right)=k^{\prime}$. Since $V_{K}(L) \otimes_{K, \lambda} k^{\prime}=V_{A}(L) \otimes_{A, \lambda} k^{\prime}$ $=\left(V_{A}(L) \otimes_{A} A^{\prime}\right) \otimes_{A^{\prime}, \lambda} k^{\prime}=V_{k^{\prime}}\left(L^{\prime}, \lambda\right)$, we get the assertion by $(1.5,(2))$.

1.11. From now on, the base field is always $\boldsymbol{C}$. Later in (4.2), (4.6) and (4.7), for a special $\boldsymbol{C}$-subspace $L$ of $\mathrm{t}^{2}$ defined over $\boldsymbol{Q}$, we shall construct using a family of twisted $\mathscr{D}$-modules, a certain $U_{A}(\mathrm{~g})$-module $M_{A}(w)^{*}(A=A(L))$ and a $U_{A}(\mathrm{~g})$-homomorphism $\varphi: M_{A}(L) \rightarrow M_{A}(w)^{*}$ satisfying the following conditions: (1.11.1) Let $j: M_{A}(w)^{*} \rightarrow M_{A}(w) * \otimes_{A} K$ be the canonical morphism and $M_{A}(w)^{*}(\mu)$ $=j^{-1}\left(\left(M_{A}(w)^{*} \otimes_{A} K\right)_{\eta-\mu}\right)$ for $\mu \subseteq Q_{+}$. Then each $M_{A}(w)^{*}(\mu)$ is a free .4-module of linite type and $M_{A}(w)^{*}=\bigoplus_{\mu \in Q_{+}} M_{A}(w)^{*}(\mu)$.

(1.11.2) Let $\varphi_{\lambda}: M(\lambda)=M_{A}(L) \otimes_{A, \lambda} C \rightarrow M_{A}(w) * \otimes_{A, \lambda} C(\lambda \in L)$ be the homomorphism induced by $\varphi$. Then $\varphi_{\lambda} \neq 0$ for any $\lambda \subseteq L$.

(1.11.3) There exists an open dense subset $L^{0}$ of $L$ with respect to the classical topology such that $M_{A}(w) * \otimes_{A, \lambda} C$ is a simple $U(\mathrm{~g})$-module for $\lambda \equiv L^{0}$.

The remainder of this section is clevoted to the proof of the following lemma.

Lemma 1.12. Assume that $L\left(\subset \mathrm{t}^{2}\right)$ is defined over $Q$. If a $U_{A}(\mathrm{~g})$-module $M_{A}(w)^{*}$ and a $U_{A}(\mathrm{~g})$-homomorphism $\varphi: M_{A}(L) \rightarrow M_{A}(w)^{*}$ satisfy (1.11.1)-(1.11.3), then $\operatorname{ch} V(L, \lambda)=\operatorname{ch}\left(M_{A}(w)^{*} \otimes_{A, \lambda} C^{\top}\right)(\lambda \equiv L)$.

1.13. Let us fix $\lambda \in E$, and an affine line $L_{1}$ of $L$ containing $\lambda$. Let $A_{1}=$ $A\left(L_{1}\right)$, and $K_{1}=K\left(L_{1}\right)$ be the quotient field of $A_{1}$. Then there are natural morphisms $A \rightarrow A_{1} \stackrel{\lambda}{\rightarrow} C$. whose composition is $\lambda: A \rightarrow C$. Let $M_{A_{1}}(w)^{*}=M_{A}(w)^{*}$ $\otimes_{A} A_{1}, M_{A_{1}}(w) *(\mu)=M_{A}(w)^{*}(\mu) \otimes_{A} A_{1}, \varphi_{1}=\varphi \otimes_{A} A_{1}$, etc. As a first step of the proof of (1.12), let us show that the natural homomorphism $M_{A_{1}}\left(L_{1}\right) \rightarrow V_{A_{1}}\left(L_{1}\right)$ induces

$$
\varphi_{1}\left(M_{A_{1}}\left(L_{1}\right)\right)=M_{A_{1}}\left(L_{1}\right) / \operatorname{ker} \varphi_{1} \longrightarrow V_{A_{1}}\left(L_{1}\right),
$$

i. e., that $\operatorname{ker} \varphi_{1} \subset M_{A_{1}}\left(L_{1}\right)_{+}$. Assume that $\operatorname{ker} \varphi_{1} \not \subset M_{A_{1}}\left(L_{1}\right)_{+}$. Take an element 
$u \equiv \operatorname{ker} \varphi_{1}$ such that $u=u_{+}+u_{0}$ with $u_{+} € M_{A_{1}}\left(L_{1}\right)_{+}$and $0 \neq u_{0} \Subset A_{1} u(c)$. Then

$$
\varphi_{1}\left(u_{0}\right)=-\varphi_{1}\left(u_{+}\right) E M_{A_{1}}(w)^{*}(0) \cap \bigoplus_{\mu \neq 0} M_{A_{1}}(w) *(\mu)=0
$$

by (1.11.1). Since $u_{0} \neq 0$, there exists $\lambda^{\prime} \equiv L_{1}$ such that the image $\bar{u}_{0}$ of $u_{0}$ by the natural homomorphism $M_{A_{1}}\left(L_{1}\right) \rightarrow M_{A_{1}}\left(L_{1}\right) \otimes_{A_{1}, \lambda}, C=M\left(\lambda^{\prime}\right)$ is non-zero. Let $\varphi^{\prime}:=\varphi_{1} \otimes_{A_{1}, \lambda}, C=\varphi \otimes_{A, \lambda}, C$. By (1.13.2), $\varphi^{\prime}\left(\bar{u}_{0}\right)=0$. Since $\bar{u}_{0}$ is a generator of $M\left(\lambda^{\prime}\right)$, this contradicts (1.11.2). Thus we get (1.13.1). Similarly, letting $\varphi_{K_{1}}=$ $\varphi_{1} \otimes K_{1}$, we get a surjective $U_{K_{1}}(\mathrm{~g})$-homomorphism

$$
M_{K_{1}}\left(L_{1}\right) / \operatorname{ker} \varphi_{K_{1}} \longrightarrow V_{K_{1}}\left(L_{1}\right) .
$$

Lemma 1.14. If $L^{0} \cap L_{1} \neq \phi$, then $M_{K_{1}}(w)^{*}=M_{A_{1}}(w)^{*} \otimes_{A_{1}} K_{1}$ is a simple $U_{K_{1}}(\mathrm{~g})$-module. (See (1.11.3) for $L^{0}$.)

Proof. Let $N$ be a $U_{K_{1}}(\mathrm{~g})$-submodule of $M_{K_{1}}(w)^{*}$. By (1.11.1), we can regard $M_{A_{1}}(w)^{*}$ as a submodule of $M_{K_{1}}(w)^{*}$. Let $N_{A_{1}}=M_{A_{1}}(w)^{*} \cap N$ and $N_{A_{1}}(\mu)$ $=M_{A_{1}}(w) *(\mu) \cap N$. For any $\mu \in Q_{+}$, there is a finite subset $L_{1}(\mu)$ of $L_{1}$ such that the quasi-coherent sheaf on $L_{1}$ obtained by localizing the $A_{1}$-module $M_{A_{1}}(w) *(\mu) / N_{A_{1}}(\mu)$ is locally free on $L_{1} \backslash L_{1}(\mu)$. (Note that a finitely generated module over the principal ideal domain $A_{1}$ is a direct sum of a free module and a torsion module.) For $\mu \in Q_{+}$, take $a(\mu) \in A_{1}$ so that $L_{1} \backslash L_{1}(\mu)=$ $\operatorname{Spec} A_{1}\left[a(\mu)^{-1}\right]$. Take $\lambda^{\prime} \equiv\left(L^{0} \cap L_{1}\right) \backslash \bigcup_{\mu \in Q_{+}} L_{1}(\mu) \neq \phi$. Then $\lambda^{\prime}$ gives an algebra homomorphism $\lambda^{\prime}: A_{1}\left[a(\mu)^{-1}\right] \rightarrow C$ for any $\mu \subseteq Q_{+}$, and

$$
\begin{aligned}
0= & \operatorname{Tor}_{1}^{A_{1}\left[a(\mu)^{-1}\right]}\left(\left(\left(M_{A_{1}}(w)^{*}(\mu) / N_{A_{1}}(\mu)\right)\left[a(\mu)^{-1}\right], C\left(\lambda^{\prime}\right)\right)\right. \\
& \longrightarrow N_{A_{1}}(\mu) \otimes_{A_{1}, \lambda}, C \longrightarrow M_{A_{1}}(w)^{*}(\mu) \otimes_{A_{1}, \lambda}, C
\end{aligned}
$$

is exact for any $\mu \equiv Q_{+}$. Any $u \in N_{A_{1}}$ can be uniquely expressed as $u=$ $\sum_{\mu \in Q_{+}} u(\mu)$ with $u(\mu) \leqq N \cap M_{K_{1}}(w)^{*}(\mu)$. On the other hand, by (1.11.1), $u \equiv N_{A_{1}}$ $\subset M_{A_{1}}(w)^{*}$ can be uniquely expressed as $\Sigma u(\mu)$ with $u(\mu) \equiv M_{A_{1}}(w)^{*}(\mu)$. Hence $u(\mu) 巨 N \cap M_{A_{1}}(w)^{*}(\mu)=N_{A_{1}}(\mu)$ and

$$
N_{A_{1}}=\bigoplus_{l \in Q_{+}} N_{A_{1}}(\mu) .
$$

By (1.14.1) and (1.14.2),

$$
0 \longrightarrow N_{A_{1}} \otimes_{A_{1}, \lambda}, C \longrightarrow M_{A_{1}}(w)^{*} \otimes_{A_{1}, \lambda}, C \longrightarrow\left(M_{A_{1}}(w)^{*} / N_{A_{1}}\right) \otimes_{A_{1}, \lambda}, C \longrightarrow 0
$$

is exact. Since $\lambda^{\prime} \boxminus L^{0}, M_{A_{1}}(w)^{*} \otimes_{A_{1}, \lambda^{\prime}} \boldsymbol{C}=M_{A}(w)^{*} \otimes_{A, \lambda} \boldsymbol{C}$ is a simple $U(\mathrm{~g})$-module. Hence $N_{A_{1}} \otimes_{A_{1}, \lambda}, \boldsymbol{C}=0$ or $\left(M_{A_{1}}(w)^{*} / N_{A_{1}}\right) \otimes_{A_{1}, \lambda^{\prime}} \boldsymbol{C}=0$. Assume that $N_{A_{1}} \otimes_{A_{1}, \lambda^{\prime}} \boldsymbol{C}=0$. Then $N_{A_{1}}(\mu) \otimes_{A_{1}, \lambda}, C=0$ for any $\mu \in Q_{+}$by (1.14.2). Since the submodule $N_{A_{1}}(\mu)$ of the free module $M_{A_{1}}(w)^{*}(\mu)$ is also $A_{1}$-free, $N_{A_{1}}(\mu) \otimes_{A_{1}, \lambda} C=0$ implies $N_{A_{1}}(\mu)$ $=0, N_{A_{1}}=0$ and hence $N=0$. Next assume that $\left(M_{A_{1}}(w)^{*} / N_{A_{1}}\right) \otimes_{A_{1}, \lambda}, \mathbb{C}=0$. Then $\left(M_{A_{1}}(w)^{*}(\mu) / N_{A_{1}}(\mu)\right) \otimes_{A_{1}, \lambda}, C=0$ by (1.11.1) and (1.14.2). Since $\left(M_{A_{1}}(w)^{*}(\mu) /\right.$ $\left.N_{A_{1}}(\mu)\right)\left[a(\mu)^{-1}\right]$ is a free $A_{1}\left[a(\mu)^{-1}\right]$-module, it implies $\left(M_{A_{1}}(w)^{*}(\mu) / N_{A_{1}}(\mu)\right)$ 
$\left[a(\mu)^{-1}\right]=0$, i. e., $M_{A_{1}}(w)^{*}(\mu)\left[a(\mu)^{-1}\right]=N_{A_{1}}(\mu)\left[a(\mu)^{-1}\right]$. Considering the $K_{1^{-}}$ subspaces of $M_{K_{1}}(w)^{*}(\mu)$ generated by the both members, we get $M_{K_{1}}(w)^{*}(\mu)=$ $M_{K_{1}}(w)^{*}(\mu) \cap N$, and hence $N=M_{K_{1}}(w)^{*}$. Therefore, $M_{K_{1}}(w)^{*}$ is a simple $U_{K_{1}}(\mathfrak{g})$ module.

Lemma 1.15. If $L^{0} \cap L_{1} \neq \phi$, then $\varphi_{K_{1}}=\varphi_{1} \otimes K_{1}: M_{K_{1}}\left(L_{1}\right) \rightarrow M_{K_{1}}(w)^{*}$ is surjective.

Proof. By (1.11.1) and (1.11.2), $\varphi_{K_{1}} \neq 0$. Since $M_{K_{1}}(w)^{*}$ is a simple $U_{K_{1}}(\mathrm{~g})$ module by (1.14), we get the assertion.

Lemma 1.16. If $L^{0} \backslash L_{1} \neq \phi$, then $M_{K_{1}}\left(L_{1}\right) / \operatorname{Ker} \varphi_{K_{1}} \underset{\rightarrow}{\rightarrow} V_{K_{1}}\left(L_{1}\right)$.

Proof. By (1.14) and (1.15), $M_{K_{1}}\left(L_{1}\right) / \operatorname{ker} \varphi_{K_{1}}$ is a simple $U_{K_{1}}(\mathrm{~g})$-module. Hence (1.13.3) is an isomorphism. (Note that $V_{A_{1}}\left(L_{1}\right)=\left(M_{A_{1}}\left(L_{1}\right)_{+} / J_{A_{1}}\left(L_{1}\right)\right) \oplus A_{1} v(c)$ and hence $V_{I_{1}}\left(L_{1}\right) \neq 0$.)

1.17. Let $M_{K}(L)(\mu)=M_{K}(L)_{\eta-\mu}$, and $M_{A}(L)(\mu)=M_{A}(L) \cap M_{K}(L)(\mu)$ for $\mu$ 三 $Q_{+}$. Let $u \equiv J_{A}(L)$ and decompose it as $u=\sum_{\mu \in Q_{+}} u(\mu)$ with $u(\mu) \Subset M_{A}(L)(\mu)$. Since $J_{A}(L)$ is a $U_{A}(\mathrm{t})$-stable and $u(\mu)$ 's belong to different weight spaces, $u(\mu) \in K J_{A}(L)$. Hence

$$
J_{A}(L) \subset \bigoplus_{\mu \in Q_{+}} K J_{A}(L) \cap M_{A}(L)(\mu) .
$$

The right side is contained in $M_{A}(L)_{+}$and stable under the actions of $.1, \mathrm{t}$ and $\mathrm{g}(\alpha)(\alpha \in R)$. Thus the right side of (1.17.1) is also a $U_{A}(\mathrm{~g})$-submodule contained in $M_{A}(L)_{+}$and hence (1.17.1) is an equality. Put $J_{A}(L)(\mu)=K J_{A}(L) \cap M_{A}(L)(\mu)$, $V_{A}(L)(\mu)=M_{A}(L)(\mu) / J_{A}(L)(\mu)$, and $V(L, \lambda)(\mu)=V(L, \lambda)_{\lambda-\mu}$ for $\lambda \in L$. Define $J_{A_{1}}\left(L_{1}\right)(\mu)$ and $V_{A_{1}}\left(L_{1}\right)(\mu)$ in the same way. Then

$$
\begin{aligned}
& J_{A}(L)=\bigoplus_{\mu \in Q_{+}} J_{A}(L)(\mu), \\
& V_{A}(L)=\bigoplus_{\mu \in Q_{+}} V_{A}(L)(\mu), \\
& V(L, \lambda)=V_{A}(L) \otimes_{A, \lambda} C=\bigoplus_{\mu \in Q_{+}} V_{A}(L)(\mu) \otimes_{A, \lambda} C, \quad \text { and } \\
& V(L, \lambda)(\mu)=V_{A}(L)(\mu) \otimes_{A, \lambda} C=V_{A_{1}}\left(L_{1}\right)(\mu) \otimes_{A_{1}, \lambda} \boldsymbol{C} .
\end{aligned}
$$

We can also see that (1.13.1) induces a surjective $U_{A_{1}}(\mathrm{~g})$-homomorphism

$$
\begin{aligned}
\varphi_{1}\left(M_{A_{1}}\left(L_{1}\right)(\mu)\right) & =\operatorname{image}\left(M_{A_{1}}\left(L_{1}\right)(\mu) \longrightarrow M_{A_{1}}\left(L_{1}\right) / \operatorname{ker} \varphi_{1}\right) \\
& \longrightarrow \operatorname{image}\left(M_{A_{1}}\left(L_{1}\right)(\mu) \longrightarrow V_{A_{1}}\left(L_{1}\right)\right)=V_{A_{1}}\left(L_{1}\right)(\mu) .
\end{aligned}
$$

Since $V_{A}(L)(\mu)$ is an -t-module of finite type, we can prove by the "Nakayama's lemma" that for any $\mu E Q_{+}$and $\lambda \equiv L$, there is a Zariski open neighbourhood $U(\mu)$ of $\lambda$ in $L$ such that 


$$
\operatorname{dim} V\left(L, \lambda^{\prime}\right)(\mu) \leqq \operatorname{dim} V(L, \lambda)(\mu)
$$

for any $\lambda^{\prime} \Leftarrow \equiv U(\mu)$. By $(1.7,(3))$, we may assume that $U(\mu)$ are defined over $\boldsymbol{Q}\langle\lambda\rangle$.

1.18. Proof of (1.12). Let $L$ be a $C$-subspace of $\mathrm{f}^{2}$ defined over $\boldsymbol{Q}, \lambda$ an element of $L$, and $U(\mu)$ as above. Take $\lambda^{\prime} \cong L^{0}$ so that tr. $\operatorname{deg}_{Q\langle\lambda\rangle} Q\langle\lambda\rangle\left\langle\lambda^{\prime}\right\rangle=$ $\operatorname{dim} L$ (cf. (1.9)). Since $U(\mu)$ are defined over $Q\langle\lambda\rangle, \lambda^{\prime} \leq U(\mu)$ for any $\mu$. Let $L_{1}$ be an affine line containing $\lambda$ and $\lambda^{\prime}$. By (1.11.2), the image of $\varphi_{\lambda^{\prime}}$ is a non-zero $U(\mathrm{~g})$-submodule of $M_{A}(w)^{*} \otimes_{A, \lambda} \boldsymbol{C}$. Since $\lambda^{\prime}=L^{0}, M_{A}(w)^{*} \otimes_{A, \lambda^{\prime}} \boldsymbol{C}$ is simple by (1.11.3). Hence $\varphi_{\lambda}$ is surjective, and $M_{A}(w)^{*} \otimes_{A, \lambda}, C$ is a simple quotient of the Verma module $M\left(\lambda^{\prime}\right)$. By (1.10), $V\left(L, \lambda^{\prime}\right)$ is also a simple quotient of the same Verma module. Hence

$$
M_{A}(w)^{*} \otimes_{A, \lambda}, C \cong V\left(L, \lambda^{\prime}\right) .
$$

By (1.15), $\varphi_{K_{1}}=\varphi_{1} \otimes_{A_{1}} K_{1}: M_{K_{1}}\left(L_{1}\right) \rightarrow M_{K_{1}}(w)^{*}$ is surjective. Since the homomorphisms induced by $\varphi_{1} \otimes_{A_{1}} K_{1}$ between the weight spaces are also surjective, $\varphi_{1}\left(M_{A_{1}}\left(L_{1}\right)(\mu)\right)$ is an $A_{1}$-lattice of the free $A_{1}$-module $M_{A_{1}}(w) *(\mu)$. Since $A_{1}$ is a principal ideal domain,

$$
\varphi_{1}\left(M_{A_{1}}\left(L_{1}\right)(\mu)\right) \cong M_{A_{1}}(w)^{*}(\mu)
$$

as $A_{1}$-modules. By (1.17.5), the surjection (1.17.6) induces a surjection $\varphi_{1}\left(M_{A_{1}}\left(L_{1}\right)(\mu)\right) \otimes_{A_{1}, \lambda} C \rightarrow V(L, \lambda)(\mu)$ for any $\mu \boxminus Q_{+}$. Thus by (1.18.2), we get the inequality

$$
\operatorname{dim}\left(M_{A_{1}}(w)^{*}(\mu) \otimes_{A_{1}, \lambda} C\right) \geqq \operatorname{dim} V(L, \lambda)(\mu) .
$$

On the other hand,

$$
\begin{array}{rlrl} 
& \operatorname{dim} V(L, \lambda)(\mu) & \\
\geqq & \operatorname{dim} V\left(L, \lambda^{\prime}\right)(\mu) & & \text { by }(1.17 .7) \\
= & \operatorname{dim}\left(M_{A}(w)^{*}(\mu) \otimes_{A_{\lambda},} C\right) & & \text { by }(1.18 .1) \\
= & \operatorname{dim}\left(M_{A}(w)^{*}(\mu) \otimes_{A_{\lambda} \lambda} C^{\prime}\right) & & \text { by }(1.11 .1) \\
= & \operatorname{dim}\left(M_{A_{1}}(w)^{*}(\mu) \otimes_{A_{1}, \lambda} C\right) . &
\end{array}
$$

Thus we get the desired equality.

Remark 1.19. (1) If $L=\{\lambda\}$, then $V(L, \lambda)=V_{k}(\lambda)$. (2) If $L=\mathrm{t}^{2}$, then $V(L, \lambda)=M_{k}(\lambda)$ for any $\lambda$. Thus our module is a generalization of Verma modules and also of their simple quotients.

Let us prove (2). By $[9,7.6 .24], M_{K}(\eta)$ is simple in this case. Hence $J_{K}(\eta)=0$. By (1.4.1), it follows that $J_{A}(L)=0, V_{A}(L)=M_{A}(L)$, and $V(L, \lambda)=$ $M_{k}(\lambda)$. 


\section{$\S 2$. Twisted $\mathscr{D}$-Modules on Homogeneous Spaces}

2.0. The purpose of this section is to review the concept of twisted ring of differential operators $\mathscr{D}_{X}(\lambda)$ due to Beilinson-Bernstein [1] (cf. (2.2)), and to define the $\mathscr{D}_{X}(\lambda)$-modules $\mathcal{O}\left(V_{0}, \lambda\right)$ (cf. (2.5)) and $H_{T}^{2}\left(X, \mathcal{O}_{X}(\lambda)\right)$ (cf. (2.9)). We also consider their 'relative versions' (cf. (2.10)-(2.13)).

2.1. First we fix some notations used in this section. For a smooth algebraic variety $X$ over the complex number field $\boldsymbol{C}$, denote the underlying complex manifold by $X^{a n}$. A morphism $f: X \rightarrow Y$ of smooth algebraic varieties is denoted by $f^{a n}$ if it is considered as a morphism between the underlying complex manifolds. For a complex manifold $\tilde{X}$, we denote the sheaf of holomorphic functions by $\mathcal{O}^{a n}=\mathcal{O}_{\tilde{X}}^{a^{n}}$. We write $\mathcal{O}_{X}^{a n}$ for $\mathcal{O}_{X^{a n}}^{a n}$. Let $\iota=\iota_{X}:\left(X^{a n}, \mathcal{O}^{a n}\right)$ $\rightarrow(X, \mathcal{O})$ be the morphism of ringed spaces induced by the identity mapping, where $\mathcal{O}=\mathcal{O}_{X}$ is the sheaf of regular functions. Let $G$ be a complex algebraic group and $g$ its Lie algebra. For an algebraic action $\sigma: G \times X \rightarrow X$, let $\sigma(g) x$ $=\sigma(g, x)$, and $(\sigma(A) f)(x)=\left.(d / d t) f\left(\sigma\left(e^{-t A}\right) x\right)\right|_{\iota=0}$ for $A \in g$ and a smooth function $f$ on $X$. Define $G$-actions $R$ and $L$ on $G$ itself by $R(g) x=x g^{-1}$ and $L(g) x=$ $g x$ for any $g, x \subseteq G$.

2.2. Twisted ring of differential operators $\mathscr{D}_{X}(\lambda)$. Let $H$ be a connected algebraic subgroup of $G, \mathfrak{h}=\operatorname{Lie}(H)$ and $\lambda: \mathfrak{h} \rightarrow C$ a character of the Lie algebra $\mathfrak{h}$. Let $F=F(\lambda)=F(\lambda, H)$ be the sheaf on $G^{a n}$ of local holomorphic functions $f$ such that $R(A) f=-\lambda(A) f$ for any $1 \Xi \mathfrak{h}$. Let $X=G / H$ and $p=p_{Y}: G \rightarrow Y=$ $G / H$ be the natural projection. Since $F$ has a $\left(p^{a n}\right)^{-1} O_{X}^{a n}$-module structure, it also has an $c_{G}^{-1} p^{-1} \Theta_{X}$-module structure. Since $L(A)(.1 \equiv \mathrm{g})$ preserves $F, F$ has a structure of $\mathrm{g}$-module. Let $\mathscr{D}_{X}(\lambda)$ be the subring of $\iota_{*} p_{*}^{a n} \underline{\operatorname{End}_{C}}(F)$ generated by the endomorphisms induced by $\mathcal{O}_{X}$ and $\mathrm{g}$. Here $\underline{\text { End }}$ is the sheaf of local endomorphisms. Then $\mathscr{D}_{X}(\lambda)$ is a twisted ring of (algebraic) differential operaturs (cf. $[19,2.3 .3]$ ). Since $\mathscr{D}_{X}(\lambda)$ is locally isomorphic to $\mathscr{D}_{X}[19,4.16]$, we can naturally generalize definitions concerning $\mathscr{D}_{X}$-modules to general $\mathscr{D}_{X}(\lambda)$ modules. We shall use the concept of characteristic variety $S S(\mathscr{M})$ of a $\mathscr{D}_{X}(\lambda)$ module $\mathscr{M}$. holonomicity, etc. without further explanation.

2.3. $\mathscr{D}_{X}(\lambda)$-module $\mathcal{O}^{a n}\left(V_{0}, \lambda\right)$. Assume that there exists an algebraic subvariety $V_{0}$ of $G$ such that $\not \mid V_{0}: V_{0} \rightarrow X(=G / H)$ is an open immersion, and let $V$ be an open neighbourhood of $V_{0}$ in $G$ with respect to the classical topology such that $p\left(V_{0}\right)=p(V)$ and each fibre of $p \mid V$ is connected and simply connected. Then the restriction $f \rightarrow f \mid V_{0}$ defines an isomorphism

$$
r=r_{V_{0}}:\left(p^{a n} \mid V\right)_{*}(F \mid V) \longrightarrow\left(p^{a n} \mid V_{0}\right)_{*} \mathcal{O}_{V_{0}}^{a n}\left(\cong \mathcal{O}_{p\left(V_{0}\right)}^{a n}\right) .
$$

Since $\mathscr{D}_{X}(\lambda) \mid p\left(V_{0}\right)$ acts on $\iota_{*}\left(p^{a n} \mid V\right)_{*}(F \mid V), \iota_{*} \mathcal{O}_{p\left(V_{0}\right)}^{a n}$ has a $\mathscr{D}_{X}(\lambda)$-module structure. 
(Here and below, we say that $\iota_{*} \mathcal{O}_{p\left(V_{0}\right)}^{a n}$ etc. is a $\mathscr{D}_{X}(\lambda)$-module instead of a $\left(\mathscr{D}_{X}(\lambda) \mid p\left(V_{0}\right)\right)$-module etc., if there is no fear of confusion.) This $\mathscr{D}_{X}(\lambda)$-module structure of $\iota_{*} \Theta_{p\left(V_{0}\right)}^{a n}$ does not depend on the choice of a neighbourhood $V$ of a given $V_{0}$, but as we shall see later in (2.7), it depends on the choice of $V_{0}$. If we need to specify this dependence, we write $\mathcal{O}^{a n}\left(V_{0}, \lambda\right)$ for $\iota_{*} \mathcal{O}_{p\left(V_{0}\right)}^{a n}$.

2.4. Let $H_{0}$ be a connected, simply connected, open neighbourhood of the identity element $e$ of $H$, and $\mathscr{H}$ the set of such open neighbourhoods $H_{0}$. For any $H_{0} \in \mathscr{K}$, a linear character $\lambda$ of the Lie algebra $\mathfrak{h}$ determines a unique holomorphic function $\lambda^{\prime}: H_{0} \rightarrow C^{<}$such that $\lambda^{\prime}(e)=1$ and $\lambda^{\prime}(x y)=\lambda^{\prime}(x) \lambda^{\prime}(y)$ whenever $x, y$ and $x y$ are contained in $H_{0}$. In the sequel, until the end of (2.9), we fix a character $\lambda$ of $\mathfrak{h}$, and write $\lambda$ for $\lambda^{\prime}$. Let $i: V_{0} \times H \rightarrow V_{0} H$ be the natural isomorphism. Then for $H_{0} \Subset \mathscr{A}, V=V_{0} H_{0}$ satisfies the condition of (2.3) and

$$
\left(i^{a n} \mid V_{0} \times H_{0}\right)^{-1}(F(\lambda, H) \mid V)=\mathcal{O}_{V_{0}}^{a n} \otimes_{C} C \lambda^{-1} .
$$

Lemma 2.5. The twisted ring of (algebraic) differential operators $\mathscr{D}_{X}(\lambda)$ preserves the subsheaf $\mathcal{O}_{p\left(V_{0}\right)}$ of $\left(_{*} \mathcal{O}_{p\left(V_{0}\right)}^{a n}\right.$. We denote this $\mathscr{D}_{X}(\lambda)$-module $\mathcal{O}_{p\left(V_{0}\right)}$ by $\mathcal{O}\left(V_{0}, \lambda\right)$ if we need to specify the dependence on $V_{0}$.

We omit the proof, since it is essentially contained in the proof of (2.12).

2.6. Dependence of $\mathcal{O}\left(V_{0}, \lambda\right)$ on $V_{0}$. If $V_{0}$ and $V_{0}^{\prime}$ are algebraic subvarieties of $G$ such that $p\left(V_{0}\right)=p\left(V_{0}^{\prime}\right)$ and, $p: V_{0} \rightarrow X(=G / H)$ and $p: V_{0}^{\prime} \rightarrow X$ are both open immersions. Then there exists a unique morphism $s: V_{0} \rightarrow H$ such that $V_{0}^{\prime}=\left\{g s(g) \mid g \subseteq V_{0}\right\}$.

Lemma 2.7. Let $V_{0}, V_{0}^{\prime}$ and $s$ be as above. The $\mathscr{D}_{X}(\lambda)$-module structure of $\mathcal{O}\left(V_{0}, \lambda\right)\left(r e s p . \mathcal{O}^{a n}\left(V_{0}, \lambda\right)\right)$ and $\mathcal{O}\left(V_{0}^{\prime}, \lambda\right)\left(\right.$ resp. $\left.\mathcal{O}^{a n}\left(V_{0}^{\prime}, \lambda\right)\right)$ are the same if and only if $\lambda_{0} \mathrm{~s}$ is locally constant on $\mathrm{s}^{-1}\left(H_{0}\right) \cap V_{0}$ for any $H_{0} \equiv \mathcal{H}$. (Note that $\mathcal{O}\left(V_{0}, \lambda\right)=$ $\mathcal{O}\left(V_{0}^{\prime}, \lambda\right)=\mathcal{O}_{p\left(V_{0}\right)}$ and $\mathcal{O}^{a n}\left(V_{0}, \lambda\right)=\mathcal{O}^{a n}\left(V_{0}^{\prime}, \lambda\right)=\iota_{*} \mathcal{O}_{p\left(V_{0}\right)}^{a n}$ as sheaves on $p\left(V_{0}\right)$.)

Proof. It is enough to consider the g-module structures on $\mathcal{O}^{a n}\left(V_{0}, \lambda\right)$ and $\mathcal{O}^{a n}\left(V_{0}^{\prime}, \lambda\right)$. Although we have assumed $V_{0}$ and $V_{0}^{\prime}$ to be algebraic subvarieties, we may assume them to be analytic subvarieties as far as we are dealing with the analytic case. Since the problem is local with respect to the classical topology, we may shrink $V_{0}$ arbitrarily. For a given $v_{0} \equiv V_{0}$, we can find $H_{0} \cong$ $\mathscr{H}$ which contains $s\left(v_{0}\right)$ and $s\left(v_{0}\right)^{-1}$. Since in a small neighbourhood of $v_{0}$, the value of $s$ is always contained in $H_{0}$, we may assume from the beginning that $s\left(V_{0}\right) \subset H_{0}$. Then $V:=V_{0} H_{0}$ is an open neighbourhood of $V_{0}$ and $V_{0}^{\prime}$. Let $A \subseteq \mathrm{g}$, $f \in \mathcal{O}_{p\left(V_{0}\right)}^{a n}=\mathcal{O}_{p\left(V_{0}^{\prime}\right)}^{a n}, r_{V_{0}}^{-1}\left(f p \mid V_{0}\right)=f_{0}$ and $r_{V_{0}^{\prime}}^{-1}\left(f p \mid V_{0}^{\prime}\right)=f_{0}^{\prime}$. (See (2.3) for $r_{V_{0}}$ and $r_{V_{0}^{\prime}}$.) Then $f_{0}\left|V_{0}=f p\right| V_{0}$ and $f_{0}(v h)=f_{0}(v) \lambda(h)^{-1}$ for any $v \Subset V_{0}$ and $h \Subset H_{0}$. Analogous 
equalities hold for $f_{0}^{\prime}$ and $V_{0}^{\prime}$. For any $v_{0} \in V_{0}$ and $t \Xi C, e^{-t A} v_{0}$ can be uniquely expressed as $e^{-t A} v_{0}=v h$ with $v=v(t) \in V_{0}$ and $h=h(t) \cong H_{0}$, if $|t|$ is sufficiently small. Then $f_{0}\left(e^{-t .4} v_{0}\right)=(f p)(v) \lambda(h)^{-1}$ and $f_{0}^{\prime}\left(e^{-t A} v_{0} s\left(v_{0}\right)\right)=f_{0}^{\prime}\left(v s(v) s(v)^{-1} h s\left(v_{0}\right)\right)=$ $(f p)(v) \lambda\left(s(v)^{-1} h s\left(v_{0}\right)\right)^{-1}$. Note that, if $|t|$ is sufficiently small, $v(t) \risingdotseq v_{0}, h(t) \risingdotseq e$ and $s(v(t))^{-1} h(t) s\left(v_{0}\right) \risingdotseq e$. Hence $s(v(t))^{-1} h(t) s\left(v_{0}\right) \subseteq H_{0}$ and $\lambda\left(s(v)^{-1} h s\left(v_{0}\right)\right)$ is defined. Moreover $s(v(t))^{ \pm 1} \risingdotseq s\left(v_{0}\right)^{ \pm 1} \equiv H_{0}$ and $h(t) s\left(v_{0}\right) \doteqdot s\left(v_{0}\right) \subseteq H_{0}$ etc. Thus $\lambda\left(s(v)^{-1} h s\left(v_{0}\right)\right)$ $=\lambda\left(s(v)^{-1}\right) \lambda\left(h s\left(v_{0}\right)\right)=\lambda\left(s(v)^{-1}\right) \lambda(h) \lambda\left(s\left(v_{0}\right)\right)=\lambda\left(s(v) s\left(v_{0}\right)^{-1}\right)^{-1} \lambda(h)$ and $f_{0}^{\prime}\left(e^{-t A} v_{0} s\left(v_{0}\right)\right)=$ $(f p)(v) \lambda(h(t))^{-1} \lambda\left(s(v(t)) s\left(v_{0}\right)^{-1}\right)$. Hence

$$
\left.\frac{d}{d t} f_{0}\left(e^{-t A} v_{0}\right)\right|_{t=0}=\left.\frac{d}{d t} f p(v(t)) \lambda(h(t))^{-1}\right|_{t=0}
$$

and

$$
\begin{aligned}
& \left.\frac{d}{d t} f_{0}^{\prime}\left(e^{-t A} v_{0} s\left(v_{0}\right)\right)\right|_{t=0} \\
& \quad=\left.\frac{d}{d t}(f p)(v(t)) \lambda(h(t))^{-1}\right|_{t=0}+\left.(f p)\left(v_{0}\right) \frac{d}{d t} \lambda\left(s(v(t)) s\left(v_{0}\right)^{-1}\right)\right|_{t=0} .
\end{aligned}
$$

Thus (2.7.1) coincides with (2.7.2) if and only if

$$
\left.\frac{d}{d t} \lambda\left(s(v(t)) s\left(v_{0}\right)^{-1}\right)\right|_{t=0}=0 .
$$

Since $\lambda\left(s(v(t)) s\left(v_{0}\right)^{-1}\right)=\lambda(s(v(t))) \lambda\left(s\left(v_{0}\right)^{-1}\right)$ if $|t|$ is sufficiently small, the condition (2.7.3) holds for any $v_{0} \Leftarrow V_{0}$ and $A \Leftarrow \mathfrak{g}$ if and only if $\lambda_{\circ} s$ is locally constant on $V_{0}$.

2.8. Let $S$ be a smooth algebraic variety, $T$ a closed subvariety of $S$, and $I=\left\{f \in \mathcal{O}_{S} \mid f \equiv 0\right.$ on $\left.T\right\}$. For an $\mathcal{O}_{S}$-module $M$, let $\Gamma_{T}(M)=\underline{\lim } \underset{\operatorname{Hom}}{\mathcal{H}_{S}}\left(\mathcal{O}_{S} / I^{m}, M\right)$. Here $\underline{H o m}$ denotes the sheaf of local homomorphisms. Let $\bar{S}$ be another smooth algebraic variety containing $S$ as an open dense subset, and $j: S \rightarrow \bar{S}$ the inclusion mapping. For an $\mathcal{O}_{\bar{S}}$-module $M$, put $\Gamma_{T}(M):=j_{*} \Gamma_{T}(M \mid S), \Gamma_{T}(\bar{S}, M):=$ $\Gamma\left(\bar{S}, \Gamma_{T}(M)\right), H_{T}^{i}(M):=H^{i}\left(R \Gamma_{T}(M)\right)$, and $H_{T}^{i}(\bar{S}, M):=H^{i}\left(R \Gamma_{T}(\bar{S}, M)\right)$. Note that $\Gamma_{T}(M)$ etc. depend only on $j^{-1} M$, and hence they can be defined also for

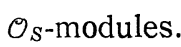

2.9. Let $V_{0}$ etc. be as before. Let $j: p\left(V_{0}\right) \rightarrow Y$ be the inclusion mapping of the open subvariety $p\left(V_{0}\right)$ of $X$, and $T$ a closed subvariety of $p\left(V_{0}\right)$. Then the $\mathscr{D}_{X}(\lambda)$-module structure of $\mathcal{O}_{p\left(V_{0}\right)}=\mathcal{O}\left(V_{0}, \lambda\right)$ induces $\mathscr{D}_{X}(\lambda)$-module structures in $H_{T}^{i}\left(\mathcal{O}_{p\left(V_{0}\right)}\right)$ and $j_{*} H_{T}^{i}\left(\mathcal{O}_{p\left(V_{0}\right)}\right)$. We shall (abusively) denote the latter sheaf by $H_{T}^{i}\left(\mathcal{O}_{X}(\lambda)\right)$, if there is no fear of confusion. Similarly, we sometimes denote the $\Gamma\left(X, \mathscr{D}_{X}(\lambda)\right)$-module $H_{T}^{i}\left(X, \mathcal{O}\left(V_{0}, \lambda\right)\right)$ by $H_{T}^{i}(X, \mathcal{O}(\lambda))$.

2.10. In the remainder of this section, we shall consider 'a relative version' of what we have considered. Let $E$ be a subvariety of $\mathfrak{h}^{2}=\operatorname{Hom}_{\text {Lie algebra }}(\mathfrak{h}, \boldsymbol{C})$. We define $G$-actions $R=R_{E}$ and $L=L_{E}$ on $G \times E$ by $R(g)\left(g^{\prime}, \lambda\right)=\left(g^{\prime} g^{-1}, \lambda\right)$ and $L(g)\left(g^{\prime}, \lambda\right)=\left(g g^{\prime}, \lambda\right)$ for $g, g^{\prime} \in G$ and $\lambda \in E$. Let $F(c)=F(c, H)$ be the sheaf 
on $(G \times E)^{a n}$ of local holomorphic functions $f$ such that $(R(A) f)(g, \lambda)=$ $-\lambda(A) f(g, \lambda)$ for $A \in \mathfrak{h}$ and $(g, \lambda) \in G \times E$. Let $p_{E}: G \times E \rightarrow X \times E(=(G / H) \times E)$ be the natural projection. Since $F(c)$ has a $\left(p_{E}^{a n}\right)^{-1} \Theta_{X \times E}^{a n}$-module structure, it also has an $\iota_{G \times E}^{1} p_{E}^{-1} \mathcal{O}_{X>E}$-module structure. Since $L(A)(A \in \mathfrak{g})$ preserves $F(c), F(c)$ has a structure of $\mathrm{g}$-module. Let $\mathscr{D}_{X, E}$ be the subring of $\iota_{*} p_{E^{*}}^{a n} \underline{E n d}_{c}(F(c))$ generated by the endomorphisms induced by $\mathcal{O}_{X \times E}$ and $\mathrm{g}$. We want to consider $\mathscr{D}_{X, E}$ as a family of twisted rings of differential operators $\mathscr{D}_{X}(\lambda)$ parametrized by $\lambda E E$.

2.11. Let $V_{0}$ and $V$ be as in (2.3). Then the restriction $f \rightarrow f \mid V_{0} \times E$ defines an isomorphism

$$
r=r_{V_{0}}:\left(p_{E}^{a n} \mid V \times E\right)_{*}(F(c) \mid V \times E) \longrightarrow\left(p_{E}^{a n} \mid V_{0} \times E\right)_{*} \mathcal{O}_{V_{0} \times E}^{a n}\left(\cong \mathcal{O}_{p\left(V_{0}\right) \vee E}^{a n}\right) .
$$

Using this isomorphism, we can define a $\mathscr{D}_{X, E}$-module stucture of $\iota_{*} \mathcal{O}_{p\left(V_{0}\right) \times E}^{a n}$ in a similar way as in (2.3). Let $H_{0} \equiv \mathscr{H}$ (cf. (2.4) for $\mathscr{H}$ ) and $c$ be the function on $H_{0} \times E$ such that $c(e, \lambda)=1$ for any $\lambda \equiv E$, where $e$ is the identity element of $H$, and $\left.(d / d t) c\left(e^{t A} h, \lambda\right)\right|_{t=0}=\lambda(A) c(h, \lambda)$ for $\lambda \in \mathfrak{h}, h \in H_{0}$ and $\lambda \in E$. (In the notation of $(2.4), c(h, \lambda)=\lambda(h)$.)

Lemma 2.12. The family of twisted rings of differential operators $\mathscr{D}_{X, E}$ preserves the subsheaf $\Theta_{p\left(V_{0}\right) \times E}$ of $\epsilon_{*} \Theta_{p\left(V_{0}\right) \times E}^{a n}$. We denote this $\mathscr{D}_{X, E}$-module $\mathcal{O}_{p\left(V_{0}\right) \times E}$ by $\mathcal{O}\left(V_{0}, c\right)$ if we need to specify the dependence on $V_{0}$.

Proof. Let $f\left(v_{0}, \lambda\right)$ be a regular function on $V_{0} \times E$, and $g: C^{\times} \rightarrow G$ be an algebraic homomorphism. If $t \doteqdot 1, g(t) v_{0}$ can be expressed as $g(t) v_{0}=v(t) h(t)$ with rational morphisms $v: C^{\times} \rightarrow V_{0}$ and $h: C^{\wedge} \rightarrow H$ which are regular at $t=1$ and satisfy $v(1)=v_{0}$ and $h(1)=e$. Since $\left.(d / d t) f\left(g(t) v_{0}, \lambda\right)\right|_{t=1}=(d / d t) f(v(t), \lambda)$ $\left.\lambda(h(t))^{-1}\right|_{t=1}=\left.(d / d t) f(v(t), \lambda)\right|_{t=1}+\left.f\left(v_{0}, \lambda\right) \cdot(d / d t) \lambda(h(t))^{-1}\right|_{t=1}$, it is enough to show that $\left.(d / d t) \lambda(h(t))^{-1}\right|_{t=1}$ is a rational function of $\left(v_{0}, \lambda\right)$. More generally, let us show that $\left.(d / d t) \lambda\left(h\left(t, v_{0}\right)\right)\right|_{t=1}$ is a rational function of $\left(v_{0}, \lambda\right)$ if $h\left(t, v_{0}\right)$ is regular in a neighbourhood of $\left\{\left(1, v_{0}\right) \mid v_{0} \in V_{0}\right\}$ and $h\left(1, v_{0}\right)=e$. Take an (algebraic) local coordinate system $\left\{x_{1}, \cdots, x_{n}\right\}$ in a neighbourhood of $e \in H$. Let $\left.(d / d t)\left(x_{i} h\right)\left(t, v_{0}\right)\right|_{t=1}=: h_{\imath}\left(v_{0}\right)$ and $\left(\partial \lambda / \partial x_{\imath}\right)(e)=: \lambda_{\imath}$. Then $\left.(d / d t) \lambda\left(h\left(t, v_{0}\right)\right)\right|_{t=1}$ $=\sum_{\imath=1}^{n} \lambda_{\imath} h_{\imath}\left(v_{0}\right)$ is a rational function of $\left(v_{0}, \lambda\right)=\left(v_{0},\left(\lambda_{1}, \cdots, \lambda_{n}\right)\right) \in V_{0} \times E$.

2.13. Take a closed subvariety $T$ of $p\left(V_{0}\right)$ as in (2.9), and let $j_{E}: p\left(V_{0}\right) \times$ $E \rightarrow X \times E$ be the inclusion mapping. The $\mathscr{D}_{X, E}$-module structure of $\mathcal{O}_{p\left(V_{0}\right) \times E}=$ $\mathcal{O}\left(V_{0}, c\right)$ induces $\mathscr{D}_{X, E}$-module structures in $H_{T \times E}^{\imath}\left(\mathcal{O}_{p\left(V_{0}\right) \times E}\right)$ and $j_{E *} H_{T \times E}^{i}\left(\mathcal{O}_{p\left(V_{0}\right) \times E}\right)$. It also induces a $\Gamma\left(X \times E, \mathscr{D}_{X, E}\right)$-module structure in $H_{T \times E}^{i}\left(X \times E, \mathcal{O}\left(V_{0}, c\right)\right)$. The evaluation of $f \sqsubseteq \Gamma\left(E, \mathcal{O}_{E}\right)$ at $\lambda \equiv E$ gives a $C$-algebra homomorphism $\Gamma\left(E, \mathcal{O}_{E}\right)$ $\rightarrow \boldsymbol{C}$, which we shall denote by $\lambda$. Then, as $\Gamma\left(X, \mathscr{D}_{X}(\lambda)\right)$-modules.

$$
H_{T \times E}^{\imath}\left(X \times E, \mathcal{O}\left(V_{0}, c\right)\right) \otimes_{\Gamma\left(E, \mathcal{O}_{E}\right), \lambda} C \cong H_{T}^{i}\left(X, \mathcal{O}_{X}(\lambda)\right) .
$$




\section{§3. Localization of $g$-modules}

3.0. In this section, we recollect results of Beilinson and Bernstein [1] concerning the localization of $\mathfrak{g}$-modules, which give a correspondence between a category of $U(\mathfrak{g})$-modules and a category of 'twisted $\mathscr{D}$-modules' on the flag manifold $X=G / B$. (Here and below, $G$ denotes again a complex reductive group as in $\S 1$.) Here we follow the exposition of Kashiwara [19], but we keep the notations of the previous sections. Thus our notations here become slightly different from those given in [19]. The twisted ring of differential operators $A_{X}(\lambda)=D_{\lambda-\rho}$ in the notation of [19] is denoted by $\mathscr{D}_{X}(\lambda)$ (cf. (2.2)) here. Also $U_{\lambda+\rho}(\mathrm{g})$ in [19] is denoted by $U(\lambda, \mathrm{g})$ (cf. (1.1)). See also [14]. Henceforth, a $\mathscr{D}$-module means a $\mathscr{D}$-module which is quasi-coherent over $\mathcal{O}$.

Lemma. 3.1. (Cf. $[19,6.2 .3]$.) For any $\lambda \in \mathrm{t}^{2}$, the natural ring homomorphism $U(\mathrm{~g}) \rightarrow \Gamma\left(X, \mathscr{D}_{X}(\lambda)\right)$ induces an isomorphism $U(\lambda-2 \rho, \mathrm{g}) \rightarrow \Gamma\left(X, \mathscr{D}_{X}(\lambda)\right)$. Hence $U(w(\lambda-\rho)-\rho, \mathfrak{g})=U(\lambda-2 \rho, \mathfrak{g})=\Gamma\left(X, \mathscr{D}_{X}(\lambda)\right)$ for any $w \cdot \equiv W$.

3.2. Let $\operatorname{Mod}_{q c}(\lambda)$ be the category of $\mathscr{D}_{X}(\lambda)$-modules $\mathscr{M}$ which are quasicoherent over $\mathcal{O}_{X}$, and $\operatorname{Mod}_{q c, 0}(\lambda)$ the subcategory of $\operatorname{Mod}_{q c}(\lambda)$ consisting of $\mathscr{M}$ satisfying the following conditions: (a) $\mathscr{M}$ is generated by global sections. (b) If a $\mathscr{D}_{Y}(\lambda)$-submodule $\mathscr{l}$ of $\mathscr{M}$ is quasi-coherent over $\mathcal{O}_{X}$ and $\Gamma(X, \Re)=0$, then $\Re=0$.

Let $\operatorname{Mod}(\lambda, \mathfrak{g})$ be the category of $U(\lambda, \mathfrak{g})$-modules. Note that $\operatorname{Mod}(w(\lambda-\rho)-$ $\rho, \mathfrak{g})=\operatorname{Mod}(\lambda-2 \rho, \mathfrak{g})$ for any $w \equiv W$. Define the functors $\Gamma: \operatorname{Mod}_{q c}(\lambda) \rightarrow \operatorname{Mod}(\lambda-$ $2 \rho, \mathfrak{g})$ and $\otimes: \operatorname{Mod}(\lambda-2 \rho, \mathfrak{g}) \rightarrow \operatorname{Mod}_{q c}(\lambda)$ by $\Gamma(\mathscr{M})=\Gamma(X, \mathscr{M})$ and $\otimes(M)=\mathscr{D}_{X}(\lambda)$ $\otimes_{U(\lambda-2 \rho, g)} M$.

Lemma 3.3. ([19, 1.5 and 6.4.2]) If $\lambda-\rho$ is anti-dominant, i.e., $\left\langle\alpha^{\nu}, \lambda-\rho\right\rangle$ $\neq 1,2, \cdots$ for any $\alpha \subseteq R_{+}$, then $\Gamma$ is an exact functor and $\Gamma \cdot \otimes=i d$. By the functors $\Gamma$ and $\otimes, \operatorname{Mod}_{q c, 0}(\lambda)$ is equivalent to $\operatorname{Mod}(\lambda-2 \rho, \mathrm{g})$.

Lemma 3.4. ([1]. Cf. $[19,6.4 .1]$.) If $\lambda-\rho$ is regular and anti-dominant, i.e., $\left\langle\alpha^{\nu}, \lambda-\rho\right\rangle \neq 0,1,2, \cdots$ for any $\alpha \Subset R_{+}$, then $\operatorname{Mod}_{q c}(\lambda)$ is equivalent to $\operatorname{Mod}(\lambda$ $-2 \rho, g)$ by $\Gamma$ and $\otimes$.

Lemma 3.5. If $\lambda-\rho$ is anti-dominant and $\mathscr{M} E \operatorname{Mod}_{q c}(\lambda)$ is holonomic, then $\operatorname{supp}(\otimes \Gamma(\mathscr{M})) \subset \operatorname{supp}(\mathscr{M})$ and $S S(\otimes \Gamma(\mathscr{M})) \subset S S(\mathscr{M})$. Here supp $($ resp. SS) denotes the support (resp. the characteristic variety).

Proof. Assume first that $\mathscr{M} \neq 0$ does not have a proper $\mathscr{D}_{X}(\lambda)$-submodule. If $\mathscr{M}$ is not generated by the global sections, then $\Gamma(X, \mathscr{M})=0$ and we get the desired inclusion. If $\mathscr{M}$ is generated by the global sections, then $\Gamma(X, \mathscr{M}) \neq 0$. Hence if a $\mathscr{D}_{X}$-submodule $\mathscr{I}$ of $\mathscr{M}$ does not have a non-zero global section, 


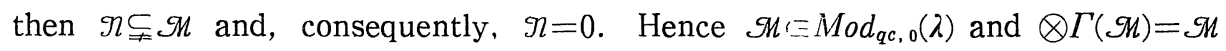
by (3.3). Thus we get the inclusion also in this case. In general, we prove the assertion by the induction on the length of $\mathscr{M}$ in $\operatorname{Mod}_{q c}(\lambda)$. Let $\mathscr{M} \in \operatorname{Mod}_{q c}(\lambda)$ be a proper submodule of $\mathscr{M}$. Since $\Gamma$ is exact by (3.3), $\otimes \Gamma(\mathscr{R}) \rightarrow \otimes \Gamma(\mathscr{M}) \rightarrow$ $\otimes \Gamma(\mathscr{M} / \mathscr{I}) \rightarrow 0$ is exact. Hence $\operatorname{supp}(\otimes \Gamma(\mathscr{M})) \subset \operatorname{supp}(\otimes \Gamma(\mathscr{I})) \cup \operatorname{supp}(\otimes \Gamma(\mathscr{M} / \mathscr{I}))$ $\subset \operatorname{supp}(\mathscr{R}) \cup \operatorname{supp}(\mathscr{M} / \mathscr{I})=\operatorname{supp}(\mathscr{M})$. The assertion concerning the characteristic varieties can be proved in the same way.

Lemma 3.6. Assume that $\lambda \in \mathrm{t}^{2}$ is anti-dominant. (1) We have $J H(M(w \lambda-\rho))$ $\subset\left\{\left[V\left(w^{\prime} \lambda-\rho\right)\right] \mid w^{\prime} \leqq w\right\}$, where $J H(-)$ denotes the set of composition factors, and [-] the isomorphism class. (See (1.2) for $V(-)$.) (2) Let $Z$ be a subset of $J H(M(w \lambda-\rho))$ which does not contain $[V(w \lambda-\rho)]$. Then $Z \subset \cup_{w^{\prime} \leqq w} J H\left(M\left(w^{\prime} \lambda\right.\right.$ $-\rho))$.

Proof. (2) follows from (1). Let us prove (1). By [3] (cf. [9, 7.6.23]), $J H(M(w \lambda-\rho))$ consists of $[V(x \lambda-\rho)]$ with the following property: There exist $\gamma_{1}, \cdots, \gamma_{n} E R_{+}$such that $w \lambda \geqq r_{\gamma_{1}} w \lambda \geqq \cdots \geqq r_{\gamma_{n}} \cdots r_{\gamma_{1}} w \lambda=x \lambda$. Put $w_{i}=r_{\gamma_{i}} \cdots r_{\gamma_{1}} w_{1}$. Since $r_{\gamma_{i}} w_{\imath-1} \lambda=w_{i-1} \lambda-\left\langle w_{i-1} \lambda, \gamma_{i}^{\sim}\right\rangle \gamma_{i} \leqq w_{i-1} \lambda,\left\langle\lambda, w_{i-1}^{-1} \gamma_{i}^{\sim}\right\rangle \in\{1,2, \cdots\}$. Since $\lambda$ is anti-dominant, $w_{i-1}^{\prime-1} \gamma_{2}$ is a negative root. Hence $w_{\imath-1}>r_{\gamma_{i}} w_{i-1}\left(=w_{i}\right)$ by $[4,2.3]$. Thus $w=w_{0}>w_{1}>\cdots>w_{n}$ and $V(x \lambda-\rho)=V\left(w_{n} \lambda-\rho\right)$.

3.7. Let $B$ and $\mathfrak{n}_{ \pm}$be as in (1.1), and $N_{ \pm}$the connected subgroup of $G$ corresponding to the Lie subalgebra $\mathfrak{n}_{ \pm}$of $\mathfrak{g}$. Let $w$ be an element of $W$. Let $p=p_{X}: G \rightarrow G / B=X$ be the natural projection, $x_{0}=p_{X}(e)$, and $X(w)=B w x_{0}$. (Here and below, we denote a representative element of $w \subset N_{G}(T) / T$ by the same letter.) Then $p \mid w N_{-}: w N_{-} \rightarrow X=G / B$ is an open immersion, and $X(w)$ is a closed subvariety of $p\left(w N_{-}\right)$which is of pure codimension $c d(w):=l\left(w_{S}\right)-$ $l(w)$. Hence, as in (2.9), for any character $\lambda$ of $\mathfrak{b}$, we can consider the $\mathscr{D}_{X}(\lambda)$ module

$$
\mathscr{X}(w)=\mathscr{X}(w, \lambda):=j_{*} H_{X(w)}^{c d(w)}\left(\mathcal{O}\left(w N_{-}, \lambda\right)\right)=H_{X(w)}^{c d(w)}\left(\mathcal{O}_{X}(\lambda)\right),
$$

which is holonomic. Here $j: p\left(w N_{-}\right) \rightarrow X$ is the inclusion mapping. By (2.7), the $\mathscr{D}_{X}(\lambda)$-module structure of $\mathscr{X}(w)=H_{X(w)}^{c d(w)}\left(\mathcal{O}_{X}(\lambda)\right)$ does not depend on the choice of the representative element of $w$. We can also consider the $\Gamma(X$, $\left.\mathscr{D}_{X}(\lambda)\right)$-module $\Gamma(X, \mathscr{X}(w, \lambda))=H_{X(w)}^{c d(w)}\left(X, \mathcal{O}_{X}(\lambda)\right)$. Recall that $\Gamma\left(X, \mathscr{D}_{X}(\lambda)\right)=U(\lambda-$ $2 \rho, \mathfrak{g})=U(u(\lambda-\rho)-\rho, \mathfrak{g})$.

Lemma 3.8. If $\lambda-\rho$ is anti-dominant, $\Gamma(X, \mathfrak{X}(w, \lambda))=M(w(\lambda-\rho)-\rho, \mathfrak{b})^{*}$.

Proof. As in [22], we can calculate the character of $\Gamma(X, \mathscr{X}(w, \lambda))$, and we get (without the assumption of anti-dominancy)

$$
\operatorname{ch} \Gamma(X, \mathscr{X}(w, \lambda))=\operatorname{ch} M(w(\lambda-\rho)-\boldsymbol{\rho}, \mathfrak{b})=\operatorname{ch} M(w(\lambda-\boldsymbol{\rho})-\boldsymbol{\rho}, \mathfrak{b})^{*}
$$


(Cf. (3.9).) Since $w(\lambda-\rho)-\rho$ is the highest among the weights of $\Gamma(X, \mathscr{X}(w$, $\lambda))^{*}$, we get a non-zero $U(\mathrm{~g})$-homomorphism $\sigma: M(w(\lambda-\rho)-\rho, \mathfrak{b}) \rightarrow \Gamma(X, \mathfrak{X}(w$, $\lambda))^{*}$ and its dual $\sigma^{*}: \Gamma(X, \mathscr{X}(w, \lambda)) \rightarrow M(w(\lambda-\rho)-\rho, \mathfrak{b})^{*}$. Let $K=\operatorname{ker} \sigma^{*}$. From the diagram

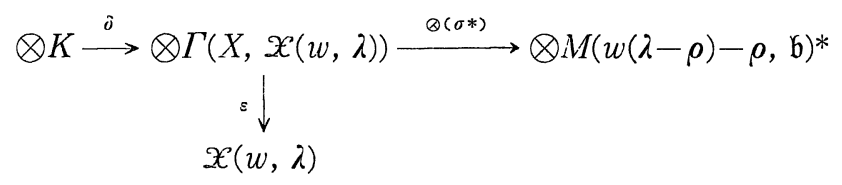

we get the following diagram.

$$
\begin{aligned}
K \stackrel{\Gamma(\grave{o})}{\rightarrow} & \Gamma(X, \mathfrak{X}(w, \lambda)) \stackrel{\sigma^{*}}{\longrightarrow} M(w(\lambda-\rho)-\rho, \mathfrak{b})^{*} \\
& \imath d=\Gamma(\varepsilon) \downarrow \\
& \Gamma(X, \mathfrak{X}(w, \lambda)) .
\end{aligned}
$$

By $(3.6,(2))$ and $(3.8 .1)$, we can show that

$$
J H(K) \subset \bigcup_{w^{\prime} \leqslant w} J H\left(M\left(w^{\prime}(\lambda-\rho)-\rho, \mathfrak{b}\right)\right)=\bigcup_{w^{\prime} \leqslant w} J H\left(\Gamma\left(X, \mathfrak{X}\left(w^{\prime}, \lambda\right)\right)\right) .
$$

By (3.5),

$$
\begin{aligned}
\operatorname{supp}(\otimes K) & \subset \bigcup_{w^{\prime} \leqq w} \operatorname{supp}\left(\otimes \Gamma\left(X, \mathscr{X}\left(w^{\prime}, \lambda\right)\right)\right) \\
& \subset \bigcup_{w^{\prime} \leqq w} \operatorname{supp}\left(\mathscr{X}\left(w^{\prime}, \lambda\right)\right)=\bigcup_{w^{\prime} \leq w} \overline{X\left(w^{\prime}\right)} .
\end{aligned}
$$

Since $\mathscr{X}(w, \lambda)=H_{X(w)}^{c d(w)}\left(\mathcal{O}_{X}(\lambda)\right)$ does not have non-zero $\mathcal{O}_{X}$-submodules whose supports are contained in $\overline{X(w)} \backslash X(w)$, (3.8.2) implies that $\varepsilon \delta=0$. Hence $\Gamma(\boldsymbol{\delta})=$ $\Gamma(\varepsilon \delta)=0$ and $\sigma^{*}$ is injective. Comparing the characters (3.8.1), we can show that $\sigma^{*}$ is an isomorphism.

Remark 3.9. In (3.8), we have assumed that $\lambda-\rho$ is anti-dominant. Here, we shall show that the assertion becomes false without this assumption.

Let $G=S L_{2}, B$ (resp. $T$ ) be the subgroup of $G$ consisting of the uppertriangular matrices (resp. the diagonal matrices), and $w=\left(\begin{array}{cc}0 & -1 \\ 1 & 0\end{array}\right)$. The mapping $G \fallingdotseq\left(\begin{array}{ll}a & b \\ c & d\end{array}\right) \rightarrow(a \infty+b) /(c \infty+d) \equiv \boldsymbol{P}^{1}$ induces an isomorphism $X=G / B \rightarrow \boldsymbol{P}^{1}$, by which we shall identify $G / B$ with $\boldsymbol{P}^{1}$. Then $G$ acts on $\boldsymbol{P}^{1}$ by $\left(\begin{array}{ll}a & b \\ c & d\end{array}\right) \cdot x=$ $(a x+b) /(c x+d)$, and $x_{0}=p_{X}(e)$ is identified with $\infty$. Note that $N_{-}=\left\{\left(\begin{array}{ll}1 & 0 \\ t & 1\end{array}\right)\right\}$ $t \equiv \boldsymbol{C}\}$.

First, let us consider $H_{X(w)}^{0}\left(X, \mathcal{O}_{X}(\lambda)\right)$. Denote the function $w\left(\begin{array}{ll}1 & 0 \\ t & 1\end{array}\right) x_{0} \rightarrow t$ by $t$. Then as vector spaces, $H_{X(w)}^{0}\left(X, \mathcal{O}_{X}(\lambda)\right)=H_{X(w)}^{0}\left(X, \mathcal{O}_{X}\right)=\boldsymbol{C}[t]$. For $\lambda \equiv \boldsymbol{C}$, 
$\left(\begin{array}{cc}a & 0 \\ 0 & a^{-1}\end{array}\right) \rightarrow a^{\lambda}$ gives a 'multi-valued character' of $T$ and induces an element of $t^{2}$, which we shall denote by the same letter $\lambda$. Then $\lambda=1$ corresponds to $\rho$. The function $p_{X}\left(w\left(\begin{array}{ll}1 & 0 \\ t & 1\end{array}\right)\right) \rightarrow t^{n}$ on $X(w)=C$, which we shall denote by $t^{n}$, is identified via $r=r_{w N_{-}}$(cf. (2.3)) with the following function $f_{n}$ in $F(\lambda, B)$ (cf. (2.2)) ;

$$
S L_{2} \ni\left(\begin{array}{ll}
a & b \\
c & d
\end{array}\right)=\left(\begin{array}{cc}
0 & -1 \\
1 & 0
\end{array}\right)\left(\begin{array}{cc}
1 & 0 \\
-a c^{-1} & 1
\end{array}\right)\left(\begin{array}{cc}
c & d \\
0 & c^{-1}
\end{array}\right) \longrightarrow\left(-a c^{-1}\right)^{n} \cdot c^{-\lambda} .
$$

Hence $\left(\left(\begin{array}{ll}0 & 1 \\ 0 & 0\end{array}\right) f_{n}\right)\left(w\left(\begin{array}{cc}1 & 0 \\ t & 1\end{array}\right)\right)=(d / d u) f_{n}\left(\left(\begin{array}{cc}1 & -u \\ 0 & 1\end{array}\right) w\left(\begin{array}{ll}1 & 0 \\ t & 1\end{array}\right)\right) !_{u=0}=n t^{n-1}$, and $\left(\begin{array}{ll}0 & 1 \\ 0 & 0\end{array}\right) t^{n}$ $=n t^{n-1}$. In the same way, we get $\left(\begin{array}{cc}1 & 0 \\ 0 & -1\end{array}\right) t^{n}=(-\lambda-2 n) t^{n}$, and $\left(\begin{array}{ll}0 & 0 \\ 1 & 0\end{array}\right) t^{n}=$ $(-\lambda-n) t^{n+1}$. Let $\tau(x)=-{ }^{t} x, C[t]^{*}$ be the dual $s l_{2}$-module (cf. (1.1)), and $\left\{e_{n}\right\}_{n \geqq 0}$ the dual basis of $\left\{t^{n}\right\}_{n \geqq 0}$. Then $\left(\begin{array}{ll}0 & 1 \\ 0 & 0\end{array}\right) e_{n}=(-\lambda-n+1) e_{n-1},\left(\begin{array}{cc}1 & 0 \\ 0 & -1\end{array}\right) e_{n}=(-\lambda-2 n) e_{n}$, and $\left(\begin{array}{ll}0 & 0 \\ 1 & 0\end{array}\right) e_{n}=(n+1) e_{n+1}$. (Here we put $e_{-1}=0$.) Thus $H_{X(w)}^{0}\left(X, \mathcal{O}_{X}(\lambda)\right)^{*}$ is isomorphic to the Verma module for any $\lambda$, whose highest weight vector is $e_{0}$ and the highest weight is $-\lambda=-\lambda \rho$.

Let us consider $H_{X(e)}^{1}\left(X, \mathcal{O}_{X}(\lambda)\right)$. Denote the function $\left(\begin{array}{ll}1 & 0 \\ t & 1\end{array}\right) x_{0} \rightarrow t$ by $t$. Then $H_{N_{-} \cdot x_{0}}^{0}\left(X, \mathcal{O}_{X}\right)=\boldsymbol{C}[t]$ and $H_{x_{0} 1}^{1}\left(X, \mathcal{O}_{X}\right)=\bigoplus_{j \geq 1} \boldsymbol{C} \boldsymbol{\delta}_{j}(t)$, where $\boldsymbol{\delta}_{j}=\left(t^{-j} \bmod \boldsymbol{C}[t]\right) \in$ $\boldsymbol{C}\left[t, t^{-1}\right] / \boldsymbol{C}[t]$. The rational function $p_{X}\left(\left(\begin{array}{ll}1 & 0 \\ t & 1\end{array}\right)\right) \rightarrow t^{n}(n \in \boldsymbol{Z})$ on $N_{-} \cdot x_{0}$, which we shall denote by $t^{-n}$, is identified via $r=r_{N}$. with the following function $f_{n}$ in $F(\lambda, B)$;

$$
S L_{2} \equiv\left(\begin{array}{ll}
a & b \\
c & d
\end{array}\right)=\left(\begin{array}{cc}
1 & 0 \\
a^{-1} c & 1
\end{array}\right)\left(\begin{array}{cc}
a & b \\
0 & a^{-1}
\end{array}\right) \longrightarrow\left(a c^{-1}\right)^{n} a^{-\lambda} .
$$

Hence $\left(\left(\begin{array}{ll}0 & 1 \\ 0 & 0\end{array}\right) f_{n}\right)\left(\left(\begin{array}{ll}1 & 0 \\ t & 1\end{array}\right)\right)=\left.(d / d u) f_{n}\left(\left(\begin{array}{cc}1 & -u \\ 0 & 1\end{array}\right)\left(\begin{array}{ll}1 & 0 \\ t & 1\end{array}\right)\right)\right|_{u=0}=(\lambda-n) t^{-n+1}$ and $\left(\begin{array}{ll}0 & 1 \\ 0 & 0\end{array}\right) \delta_{n}$ $=(\lambda-n) \boldsymbol{\delta}_{n-1}$. In the same way, we get $\left(\begin{array}{cc}1 & 0 \\ 0 & -1\end{array}\right) \delta_{n}=(\lambda-2 n) \delta_{n}$, and $\left(\begin{array}{ll}0 & 0 \\ 1 & 0\end{array}\right) \delta_{n}=$ $n \delta_{n+1}$. Let $\left\{e_{n}\right\}_{n \geq 1}$ be the dual basis of $\left\{\delta_{n}\right\}_{n \geqq 1}$. Then $\left(\begin{array}{ll}0 & 1 \\ 0 & 0\end{array}\right) e_{n}=(n-1) e_{n-1}$, $\left(\begin{array}{cc}1 & 0 \\ 0 & -1\end{array}\right) e_{n}=(\lambda-2 n) e_{n}$, and $\left(\begin{array}{ll}0 & 0 \\ 1 & 0\end{array}\right) e_{n}=(\lambda-n-1) e_{n+1}$. (Here we put $e_{0}=0$.) Thus $H_{X(e)}^{1}\left(X, \mathcal{O}_{X}(\lambda)\right)^{*}$ is isomorphic to the Verma module if and only if $\lambda-j \neq 0$ for $j=2,3,4, \cdots$, i. e., $\lambda-\rho$ is anti-dominant. (For any $\lambda, H_{X(e)}^{1}\left(X, \mathcal{O}_{X}(\lambda)\right.$ ) is the Verma module.)

§4. The $U_{A}(\mathrm{~g})$-module $M_{A}(w)^{*}$

4.0. Let $\lambda_{c}$ be a $W_{I}$-invariant character of $\mathrm{t}, \lambda_{d}$ the lowest weight of a 
finite dimensional irreducible $P$-module, $\lambda=\lambda_{c}+\lambda_{d}$, and $w \in\left(W / W_{I}\right)_{l}$. In this section, we construct a certain $U_{A}(\mathfrak{g})$-module $M_{A}(w)^{*}$ and a $U_{A}(\mathfrak{g})$-homomorphism $\varphi: M_{A}(L) \rightarrow M_{A}(w)^{*}$, which satisfy the conditions (1.11.1) and (1.11.2). Later, in $\S 6$, we shall show that $M_{A}(w)^{*}$ also satisfies the condition (1.11.3), and shall calculate the character of $V(w, \lambda, \mathfrak{p})$ using (1.12). In this section, we fix a subset $I$ of $S$. In order to make the account easier to read, first we consider the case where $\lambda_{d}=0$, and at the end of this section, indicate how to generalize it.

4.1. Naturally identify $t_{I}^{2}$ with the set $\mathfrak{p}^{2}$ of characters of the Lie algebra p. Let $\lambda_{c} \subseteq \mathrm{t}_{I}^{2}=\mathfrak{p}^{2}=: E, \lambda_{d}$ be as in (4.0) and $\lambda=\lambda_{c}+\lambda_{d}$. Put $L=L\left(w, \lambda_{d}, \mathfrak{p}\right)=$ $\left\{w\left(\lambda_{c}^{\prime}+\lambda_{d}-\rho\right)-\rho \mid \lambda_{c}^{\prime} \in E\right\}, A=A(L), A^{\prime}=A(E)$, and $K^{\prime}$ the quotient field of $A^{\prime}$. Since for our argument here, it is more convenient to consider $E$ instead of $L$, we construct a $U_{A^{\prime}}(\mathrm{g})$-module $M_{A^{\prime}}(w)^{*}$ and a $U_{A^{\prime}}(\mathrm{g})$-homomorphism $\varphi^{\prime}$ : $M_{A^{\prime}}\left(w\left(c^{\prime}+\lambda_{d}-\rho\right)-\rho\right) \rightarrow M_{A^{\prime}}(w)^{*}$ such that $M_{A^{\prime}}(w)^{*} \otimes_{A^{\prime}} A$ and $\varphi^{\prime} \otimes_{A^{\prime}} A$ satisfy the conditions (1.11.1)-(1.11.3). Here $c^{\prime} \in \operatorname{Hom}_{C}\left(\mathrm{t}, A^{\prime}\right)=\operatorname{Hom}_{A^{\prime}}\left(\mathrm{t}_{A^{\prime}}, A^{\prime}\right)$ is the natural character and $A$ is considered as an $A^{\prime}$-algebra by the isomorphism induced by $E \equiv \lambda_{c}^{\prime} \rightarrow w\left(\lambda_{c}^{\prime}+\lambda_{d}-\rho\right)-\rho \subseteq L$. Let us write out the conditions corresponding to (1.11.1)-(1.11.3), which $M_{A^{\prime}}(w)^{*}$ and $\varphi^{\prime}$ should satisfy.

(1.11.1') Let $j: M_{A^{\prime}}(w)^{*} \rightarrow M_{A^{\prime}}(w)^{*} \otimes_{A^{\prime}} K^{\prime}$ be the canonical morphism and $M_{A^{\prime}}(w)^{*}(\mu)=j^{-1}\left(\left(M_{A^{\prime}}(w)^{*} \otimes_{A^{\prime}} K^{\prime}\right)_{w\left(\eta^{\prime}+\lambda_{d^{-}} \rho\right)-\rho-\mu}\right)$ for $\mu=Q_{+}$. Then each $M_{A^{\prime}}(w)^{*}(\mu)$ is a free $A^{\prime}$-module of finite type and $M_{A^{\prime}}(w)^{*}=\bigoplus_{\mu \in Q_{+}} M_{A^{\prime}}(w)^{*}(\mu)$. Here $\eta^{\prime}=$ $c^{\prime} \otimes_{A^{\prime}} K^{\prime}$.

$\left(1.11 .2^{\prime}\right)$ Let $\varphi_{\lambda_{c}^{\prime}}^{\prime}: M\left(w\left(\lambda_{c}^{\prime}+\lambda_{d}-\rho\right)-\rho, \mathfrak{b}\right) \rightarrow M_{A^{\prime}}(w)^{*} \otimes_{A, \lambda_{c}^{\prime}} \boldsymbol{C}\left(\lambda_{c}^{\prime} \equiv \mathrm{t}_{I}^{\circ}\right)$ be the homomorhism induced by $\varphi^{\prime}$. Then $\varphi_{\lambda_{c}^{\prime}}^{\prime} \neq 0$ for any $\lambda_{c}^{\prime}=\mathrm{t}_{I}^{2}$.

$\left(1.11 .3^{\prime}\right)$ There exists an open clense subset $\mathrm{t}_{0}^{2}$ of $\mathrm{t}_{I}^{2}$ with respect to the classical topology such that $M_{A^{\prime}}(w)^{*} \otimes_{A^{\prime}, \lambda_{c}^{\prime}} C$ is a simple $U(\mathrm{~g})$-module for $\lambda_{c}^{\prime} \in \mathrm{t}_{0}^{\nu}$.

Henceforth, we shall exclusively consider $E$ as the parameter space, and we write $A, K, \varphi, c$, $\eta$, etc. for $A^{\prime}, K^{\prime}, \varphi^{\prime}, c^{\prime}, \eta^{\prime}$, etc. for the simplicity of notation. For $L=L\left(w, \lambda_{d}, \mathfrak{p}\right)=w\left(\mathfrak{p}^{2}+\lambda_{d}-\boldsymbol{\rho}\right)-\boldsymbol{\rho}$, we put $M_{A}\left(w\left(c+\lambda_{d}-\boldsymbol{\rho}\right)-\boldsymbol{\rho}\right):=$ $M(L), J_{A}\left(w, c+\lambda_{d}, \mathfrak{p}\right):=J(L), V_{A}\left(w, c+\lambda_{d}, \mathfrak{p}\right):=V(L)$,

$$
V(w, \lambda, \mathfrak{p}):=V(L, w(\lambda-\boldsymbol{\rho})-\boldsymbol{\rho})
$$

$\eta:=c \otimes K, V_{K}\left(w, \eta+\lambda_{d}, \mathfrak{p}\right):=V_{K}(L)$, etc. (Cf. (1.3).)

4.2. The $U_{A}(\mathrm{~g})$-module $M_{A}(w)$. Let $X=G / B$ and $Y=G / P$. Let $p_{X}: G \rightarrow$ $X, p_{Y}: G \rightarrow Y$ and $q: X \rightarrow Y$ be the natural projections, $x_{0}=p_{X}(e), y_{0}=p_{Y}(e)$, where $e$ is the identity element of $G, X(w)=B w x_{0}$ and $Y(w)=B w y_{0}$ for $w \subseteq W$. Let $w \subseteq\left(W / W_{I}\right)_{l}$. Then $Y(w)$ is a closed subvariety of $p_{Y}\left(w U_{-}\right)=w U_{-} \cdot y_{0}$ of pure codimension $c d(w)=l\left(w_{s}\right)-l(w)$. (Note that $X(w)$ is an open subset of $q^{-1} Y(w)$, and is of pure codimension $c d(w)$ in $X$.) Let $\mathscr{D}_{Y, E}$ be as in (2.10). Then as in (2.13), we can consider the $\Gamma\left(Y \times E, \mathscr{D}_{Y, E}\right)$-module

$$
M_{A}(w):=H_{Y(w) \times E}^{c d(w)}\left(Y \times E, \mathcal{O}\left(w U_{-}, c\right)\right)=H_{Y(w)}^{c d(w)}\left(Y, \mathcal{O}_{Y}\right) \otimes_{c} A .
$$


Since $\mathscr{D}_{Y, E}$ is generated by the operators induced by $g$ and $\mathcal{O}_{Y \times E}$ (cf. (2.10)), we have natural morphisms $U(\mathfrak{g}) \rightarrow \Gamma\left(Y \times E, \mathscr{D}_{Y, E}\right)$ and $A=\Gamma\left(E, \mathcal{O}_{E}\right) \rightarrow \Gamma\left(Y \times E, \mathcal{O}_{Y \times E}\right)$ $\rightarrow \Gamma\left(Y \times E, \mathscr{D}_{Y, E}\right)$, by which $M_{A}(u)$ becomes a $U_{A}(\mathrm{~g})$-module.

4.3. A free $A$-basis of $M_{A}(w)$. The natural $G$-action on $Y$ induces actions of the maximal torus $T$ on $Y(w)=B w y_{0}$ and $w U_{-} \cdot y_{0}$. Let $\mathfrak{u}_{w}=\bigoplus_{\alpha \in R_{+} \cap w\left(R_{-} \backslash R_{I}\right)} \mathfrak{g}(\alpha)$ and $w\left(\mathfrak{u}_{-}\right)=\bigoplus_{\alpha \in w\left(R_{-} \backslash R_{I}\right)} g(\alpha)$. By the isomorphism $x \rightarrow(\exp x) w y_{0}$, the pair $\left(w\left(\mathfrak{u}_{-}\right)\right.$, $\left.\mathfrak{l t}_{w}\right)$ is isomorphic to $\left(w U_{-} \cdot y_{0}, B w y_{0}\right)$ including the natural $T$-actions. Hence

$$
H_{Y(w)}^{c d(w)}\left(Y, \mathcal{O}_{Y}\right) \cong\left({ }_{\alpha \in R_{+} \cap} \bigotimes_{w\left(R_{-} \backslash R_{I}\right)} \Gamma(\mathrm{g}(\alpha), \mathcal{O})\right) \otimes\left({ }_{\alpha \in w\left(R_{-} \backslash R_{I}\right) \backslash R_{+}} H_{101}^{1}(\mathrm{~g}(\alpha), \mathcal{O})\right) .
$$

We can get a $\boldsymbol{C}$-basis of the left hand side of (4.3.1) by using the expression of the right hand side as follows. The exact sequence

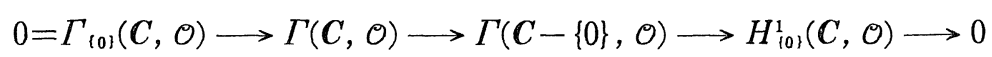

can be identified with the exact sequence

$$
0 \longrightarrow C[x] \longrightarrow C\left[x, x^{-1}\right] \longrightarrow H_{101}^{1}(C, \mathcal{O}) \longrightarrow 0 .
$$

Let $\left((d / d x)^{n} x^{-1} \bmod C[x]\right)=: \delta^{(n)}(x)$. Then

$$
\Gamma(\boldsymbol{C}, \mathcal{O})=\bigoplus_{n \geq 0} \boldsymbol{C} x^{n} \quad \text { and } \quad H_{101}^{1}(\boldsymbol{C}, \mathcal{O})=\bigoplus_{n \geq 0} \boldsymbol{C} \delta^{(n)} .
$$

Let $u_{\alpha}$ be a linear coordinate function on $g(\alpha)$ and $\delta_{\alpha}^{(n)}=\delta^{(n)}\left(u_{\alpha}\right)$. As is seen from (4.3.1) and (4.3.2), the set of the elements of the form

$$
\left(\prod_{\alpha \in R_{+} \cap} \prod_{w\left(R_{-} \backslash R_{I}\right)} u_{\alpha}^{n(\alpha)}\right) \times\left(\prod_{-\alpha \in w\left(R_{-} \backslash R_{I}\right) \backslash R_{+}} \delta_{-\alpha}^{(n(\alpha))}\right)
$$

with $n(\alpha) \geqq 0$ gives a $C$-basis of $H_{Y(w)}^{c d(w)}\left(Y, O_{Y}\right)$. It also gives a free $A$-basis of $M_{A}(w)$.

Lemma 4.4. Let $v$ be the element (4.3.3). Then

$$
H v=\left\langle w(c-\rho)-\rho-\sum_{\alpha \in R_{+} \backslash w R_{I}} n(\alpha) \alpha, H\right\rangle v \quad \text { for } H \in \mathrm{t} .
$$

Proof. The element (4.3.3) is a weight vector of the weight

$$
\begin{aligned}
& w c-\sum_{\alpha \in R_{+} \cap w\left(R_{-} \backslash R_{I}\right)} n(\alpha) \alpha+\sum_{a \in R_{+} \cap} \sum_{w\left(R_{+} \backslash R_{I}\right)}(n(\alpha)+1)(-\alpha) \\
= & w c-\sum_{a \in R_{+} \cap w\left(R_{-} \backslash R_{I}\right)} \alpha-\sum_{\alpha \in R_{+} \backslash w R_{I}} n(\alpha) \alpha .
\end{aligned}
$$

Since $w \in\left(W / W_{I}\right)_{l}$, we have $w\left(R_{+} \cap R_{I}\right) \subset R_{-}$, and hence

$$
\sum_{\alpha \in R_{+} \cap w\left(R_{+} \backslash R_{I}\right)} \alpha=\sum_{a \in R_{+} \cap w R_{+}} \alpha=\rho+w \rho .
$$

By (4.4.1) and (4.4.2), we get the assertion.

Corollary 4.5. For $\lambda \in \mathrm{I}_{I}^{\llcorner}$and $w 巨\left(W / W_{I}\right)_{l}$, 


$$
\operatorname{ch}\left(M_{A}(w) \otimes_{A, \lambda} C\right)=e^{w(\lambda-\rho)-\rho} \prod_{a \in R_{+} \backslash w R}\left(1-e^{-a}\right)^{-1} .
$$

4.6. For $w \in\left(W / W_{I}\right)_{l}$ and $\mu \Subset Q_{+}$, let $M_{A}(w)(\mu)=\left\{v \in M_{A}(w) \mid H v=\langle w(c-\rho)\right.$ $-\rho-\mu, H>v$ for $\left.H \equiv \mathfrak{t}^{2}\right\}$. By (4.3) and (4.4), $M_{A}(w)=\bigoplus_{\mu \in Q_{+}} M_{A}(w)(\mu)$ and each $M_{A}(w)(\mu)$ is a free $A$-module of finite type. Let $M_{A}(w)^{*}(\mu)=\operatorname{Hom}_{A}\left(M_{A}(w)(\mu), A\right)$, and $M_{A}(w)^{*}=\oplus_{\mu \in Q_{+}} M_{A}(w)^{*}(\mu)$. Then $M_{A}(w)^{*}$ has a natural $U_{A}(\mathrm{~g})$-module structure and

$$
\operatorname{ch}\left(M_{A}(w) * \otimes_{A, \lambda} \boldsymbol{C}\right)=\operatorname{ch}\left(M_{A}(w) \otimes_{A, \lambda} \boldsymbol{C}\right)=e^{w(\lambda-o)-\rho} \prod_{a \in R_{+} \backslash w R_{I}}\left(1-e^{-a}\right)^{-1}
$$

for $\lambda \in \mathrm{t}_{I}^{2}$. Obviously $M_{A}(w)^{*}$ satisfies the condition $\left(1.11 .1^{\prime}\right)$ of (4.1).

4.7. We have a $U_{A}(\mathfrak{b})$-homomorphism $A(w(c-\rho)-\rho) \rightarrow M_{A}(w)^{*}$ whose image is the weight space $\left.M_{A}(w)^{*}(0)=M_{A}(w)_{w(c-\rho)-\rho}^{*} \cong A\right)$. This homomorphism incluces a $U_{A}(\mathfrak{g})$-homomorphism $\varphi: M_{A}(w(c-\rho)-\rho, \mathfrak{b}) \rightarrow M_{A}(w)^{*}$. Obviously, $\varphi$ satisfies the condition $\left(1.11 .2^{\prime}\right)$ of (4.1). The condition (1.11.3') will be proved in $\S 6$.

4.8. Let us explain how to generalize the argument of this section to the case where $\lambda_{d}$ is not necessarily zero. We keep the notations of (4.2). Let $\lambda_{d}$ be the lowest weight of a finite dimensional irreducible $P$-module, and $\mathcal{O}_{X}\left(\lambda_{d}\right)$ the line bundle on $X$ consisting of (local) regular functions $f$ such that $f(g b)=f(g) \lambda_{d}(b)^{-1}$ for $g \in G$ and $b \in B$. Let $\lambda_{c} \subseteq \mathrm{t}_{I}^{2}$ and $\lambda=\lambda_{c}+\lambda_{d}$. (Note that the lowest weight $\lambda$ of a finite dimensional irreducible $\mathfrak{p}$-module can be always expressed in this way.) Then $q^{*} \mathcal{O}\left(w U_{-}, \lambda_{c}\right) \otimes_{\mathcal{O}_{X}} \mathcal{O}_{X}\left(\lambda_{d}\right)$ has a natural $\mathscr{D}_{X}(\lambda)$ module structure, which induces a $\Gamma\left(X, \mathscr{D}_{Y}(\lambda)\right)$-module structure on

$$
\begin{aligned}
& H_{q^{-1}(w)}^{c d(w)}\left(q^{-1}\left(w U_{-} \cdot y_{0}\right), q^{*} \mathcal{O}\left(w U_{-}, \lambda_{c}\right) \otimes_{\left.\mathcal{O}_{X} \mathcal{O}_{X}\left(\lambda_{d}\right)\right)}\right. \\
= & H_{Y(w)}^{c d(w)}\left(w U_{-} \cdot y_{0}, \mathcal{O}\left(w U_{-}, \lambda_{c}\right) \otimes_{\mathcal{O}_{Y}} q_{*} \mathcal{O}_{X}\left(\lambda_{d}\right)\right) \\
= & H_{Y(w)}^{c d(w)}\left(Y, q_{*} \mathcal{O}_{X}\left(\lambda_{d}\right)\right), \quad \text { (as a vector space). }
\end{aligned}
$$

(Note that $R q_{*} \mathcal{O}\left(\lambda_{d}\right)=q_{*} \mathcal{O}\left(\lambda_{d}\right)$ by the Borel-Weil theory [5].) "Varying $\lambda_{c} \in \mathrm{t}_{I}^{2}$ in (4.8.1)", we get a $\Gamma\left(X \times E, \mathscr{D}_{X, E}\right)$-module

$$
M_{A}(w):=H_{Y(w)}^{c d(w)}\left(Y, q_{*} \mathcal{O}_{X}\left(\lambda_{d}\right)\right) \otimes_{C} \cdot 4,
$$

which has also a $U_{A}(\mathrm{~g})$-module structure as in (4.2). Let $\left\{f_{\imath}\right\}$ be a basis of the $P$-module $\Gamma\left(P / B, \mathcal{O}_{X}\left(\lambda_{d}\right)\right)$ consisting of weight vectors, and $\mu_{i}$ the weight of $f_{\imath}$. Then the functions $w U_{-} \cdot P \ni w u p \rightarrow f_{\imath}(p)$, which we shall denote by $f_{\imath}$, give a $\Gamma\left(w U_{-} \cdot y_{0}, \mathcal{O}_{Y}\right)$-basis of $\Gamma\left(w U_{-} \cdot y_{0}, q_{*} \mathcal{O}_{X}\left(\lambda_{d}\right)\right)$. Let $V_{I}\left(\lambda^{\prime}\right)$ be the irreducible $\mathfrak{p}$-module with the lowest weight $\lambda^{\prime}$. Since $\Gamma\left(P / B, \mathcal{O}_{Y}\left(\lambda_{d}\right)\right) \cong V_{I}\left(\lambda_{d}\right)$ (cf. (9.2) below), we have

$$
\operatorname{ch}\left(M_{A}(w) \otimes_{A, \lambda_{c}} \boldsymbol{C}\right)=e^{-w \rho-\rho} \cdot w\left(\operatorname{ch} V_{I}(\lambda)\right) \prod_{\alpha \in R_{+} \backslash w R_{I}}\left(1-e^{-a}\right)^{-1},
$$


where $w\left(e^{\lambda^{\prime}}\right)=e^{w^{\prime \prime}}$ etc. (As is indicated by the calculation

$$
\begin{aligned}
\left(u_{\alpha}^{n} f_{i}\right)\left(t^{-1} x_{\alpha}(u) w p, \lambda_{c}\right) & =\left(u_{\alpha}^{n} f_{i}\right)\left(x_{\alpha}\left(\alpha(t)^{-1} u\right) w \cdot w^{-1} t^{-1} w p, \lambda_{c}\right) \\
& =\left(\alpha(t)^{-n} u^{n} \cdot \lambda_{c}\left(w^{-1} t w\right) \cdot \lambda_{c}(p)^{-1}\right) \cdot\left(\mu_{\imath}\left(w^{-1} t w\right) f_{\imath}(p)\right) \\
& =\left(w \mu_{\imath}\right)(t) \cdot\left(w \lambda_{c}-n \alpha\right)(t) \cdot\left(u_{\alpha}^{n} f_{\imath}\right)\left(x_{\alpha}(u) w p, \lambda_{c}\right),
\end{aligned}
$$

the character (4.8.2) is the same as the case $\lambda_{d}=0$ except for the contribution from the factor $\left(w \mu_{i}\right)(t)$, which amounts to $w\left(\operatorname{ch} V_{I}(\lambda)\right)$.) Since $w \subseteq\left(W / W_{I}\right)_{l}$, the highest weight of $M_{A}(w) \otimes_{A, \lambda_{C}} C$ is $w(\lambda-\rho)-\rho$. As in (4.6) and (4.7), we can construct a $U_{A}(\mathrm{~g})$-module $M_{A}(w)^{*}$ and a $U_{A}(\mathrm{~g})$-homomorphism $\varphi: M_{A}(w(c+$ $\left.\left.\lambda_{d}-\rho\right)-\rho, \mathfrak{b}\right) \rightarrow M_{A}(w)^{*}$ which satisfy $\left(1.11 .1^{\prime}\right)$ and $\left(1.11 .2^{\prime}\right)$.

\section{§5. Stability of the Submodule Lattice by a Smooth Pull-back}

5.1. Let $X$ be a smooth algebraic variety over the complex number field $C$, and $\mathcal{O}=\mathcal{O}_{X}$ the sheaf of regular functions on $X$. Let $Y$ be another smooth algebraic variety and $f: X \rightarrow Y$ a smooth morphism. Let $\mathscr{D}_{X}$ be the sheaf of algebraic differential operators on $X$ and $\mathscr{D}_{f}$ the subring of $\mathscr{D}_{X}$ generated by $\mathcal{O}_{X}$ and the tangent vector fields which are tangent to the fibres of $f$. Let $M^{\prime}$ be an $\mathcal{O}_{Y}$-module and $M=f^{*} M^{\prime}=\mathcal{O}_{X} \otimes_{f-1 \mathcal{O}_{Y}} f^{-1} M^{\prime}$. Then $M$ has a natural $\mathscr{D}_{f^{-}}$ module structure. The purpose of this section is to prove the following proposition.

Proposition 5.2. (1) Let $N$ be $a \mathscr{D}_{f}$-submodule of $M=f^{*} M^{\prime}$ which is quasicoherent as an $\mathcal{O}_{X}$-module. For any $p \in X$, there exists an open neighbourhood $U$ of $p$ and a uniquely determined $\mathcal{O}_{f(U)}$-submodule $N^{\prime}(U)$ of $M^{\prime} \mid f(U)$ such that $N \mid U=(f \mid U)^{*} N^{\prime}(U)$. (Note that $f$ is an open mapping.)

(2) Assume that $f$ is surjective and the fibres of $f$ are connected. Then there is a uniquely determined $\mathcal{O}_{Y}$-submodule $N^{\prime}$ of $M^{\prime}$ such that $N=f^{*} N^{\prime}$.

Remark 5.3. Since $f$ is smooth, $\mathcal{O}_{X, p}$ is faithfully flat over $\left(f^{-1} \mathcal{O}_{Y}\right)_{p}=$ $\mathcal{O}_{Y, f(p)}$. Hence for any $\mathcal{O}_{Y}$-submodule $N^{\prime}$ of $M^{\prime}, f^{*} N^{\prime}$ is an $\mathcal{O}_{X}$-submodule of $f^{*} M^{\prime}$, and, by [7. Chap. 1, $\S 3$, Prop. 9], $f^{-1} N^{\prime}$ is a subsheaf of $f^{*} N^{\prime}$.

Corollary 5.4. Assume that $f: X \rightarrow Y$ is a smooth surjective morphism whose fibres are connected. Let $M^{\prime}$ be a quasi-coherent $\mathcal{O}_{Y}$-module, $M=f^{*} M^{\prime}, S(M)$ the set of $\mathscr{D}_{f}$-submodules of $M$ which are quasi-coherent as $\mathcal{O}_{X}$-modules, and $S\left(M^{\prime}\right)$ the set of quasi-coherent $\mathcal{O}_{Y}$-submodules of $M^{\prime}$. Then $N^{\prime} \rightarrow f^{*} N^{\prime}$ defines a bijection $S\left(M^{\prime}\right) \rightarrow S(M)$.

5.5. Let $y=\left(y_{1}, \cdots, y_{r}\right)$ be a local coordinate system of $Y$ at $q=f(p)$. If there is no fear of confusion, we regard $\mathcal{O}_{Y, q}$ as a subring of $\mathcal{O}_{X, p}$ by $f^{*}$. Especially, we identify $y_{\imath} \in \mathcal{O}_{Y, q}$ with $f^{*} y_{\imath}=y_{\imath} \circ f \in \mathcal{O}_{X, p}$. Choose $z_{1}, \cdots, z_{s}$ 
$\mathcal{O}_{X, p}$ so that $x=\left(y_{1}, \cdots, y_{r} ; z_{1}, \cdots, z_{s}\right)$ gives a local coordianate system of $X$ at $p$. l'or $v=\left(v_{1}, \cdots, v_{s}\right) \in \boldsymbol{Z}^{s}$, we write $v \geqq 0$ if $v_{\imath} \geqq 0$ for any $i$, and $v>0$ if $v \geqq 0$ and $v \neq 0$. If $v \in \boldsymbol{Z}^{s}$ and $v \geqq 0$, we set $z(v)=\Pi_{i=1}^{s} z_{i}^{v_{2}} / v_{\imath}$ ! and $\partial^{v}=\partial_{z}^{v}=\left(\partial / \partial z_{1}\right)^{v_{1}}$ $\cdots\left(\partial / \partial z_{s}\right)^{v_{s}}$. If $v \geq 0$, we set $z(v)=0$. Then $\partial^{v} z(w)=z(w-v)$.

5.6. Assume that we are given an $n$-tuple $A(x)=\left(a_{1}(x), \cdots, a_{n}(x)\right) \in \mathcal{O}_{x, p}^{n}$. Consider its power series expansion with respect to $z ; A(x)=\sum_{v \geq 0} A_{v}(y) z(v)$ with $A_{v}(y) \equiv \mathcal{O}_{Y, q}^{n}$. Set $J=\sum_{v \geqq 0} \mathcal{O}_{Y, q} A_{\imath}(y)\left(\subset \mathcal{O}_{Y, q}^{n}\right)$, and let $\left\{B_{1}(y), \cdots, B_{g}(y)\right\}$ be a minimal generating system of the $\mathcal{O}_{Y^{r}, q}$-module $J$. Then $A_{v}(y)$ 's can be expressed as

$$
A_{v}(y)=\sum_{i=1}^{g} c_{v, \imath}(y) B_{\imath}(y)
$$

with some $c_{v, \imath}(y) \equiv \mathcal{O}_{Y, q}$. Set

$$
c_{\imath}(x)=\sum_{v \geq 0} c_{v, \imath}(y) z(v) .
$$

Then $c_{\imath}(x) \subseteq \hat{\mathcal{O}}_{X, p}$, where $\hat{\mathcal{O}}_{X, p}$ is the completion of the local ring $\mathcal{O}_{X, p}$ with respect to its maximal ideal $m_{X, p}$. Moreover $c_{\imath}=c_{\imath}(x)(1 \leqq i \leqq g)$ satisfy the system of linear equations with coefficients in $\mathcal{O}_{X, p}$;

$$
A(x)=\sum_{i=1}^{g} c_{i} B_{i}(y) \text {. }
$$

Since (5.6.3) has a solution in $\hat{\mathcal{O}}_{X, p}$, and since $\hat{\mathcal{O}}_{X, p}$ is faithfully flat over $\hat{\mathcal{O}}_{X, p}$, (5.6.3) has a solution in $\mathcal{O}_{X, p}$ [7, Chap. $1, \S 3$, Prop. 13]. Hence we may take $c_{\imath}(x)$ in $\mathcal{O}_{X, p}$. (Note that, if we define $c_{v, \imath}(y)$ 's as the coefficients of the power series expansion (5.6.2) of $c_{\imath}(x)$, the equality (5.6.1) holds.) Since $\left\{A_{v}(y) \mid v \geqq 0\right\}$ and $\left\{B_{\imath}(y) \mid 1 \leqq i \leqq g\right\}$ are both generators of $J, B_{\imath}(y)$ 's can be expressed as $B_{\imath}(y)=\sum_{v \geq 0} d_{i, v}(y) A_{v}(y)$ (a finite sum) with some $d_{i, v}(y) \equiv \mathcal{O}_{Y, q}$. Set $K=\mathcal{O}_{X, p} J$ and $P_{\imath}=P_{\imath}\left(y, \partial_{z}\right)=\sum_{v \geqq 0} d_{\imath, v}(y) \partial_{z}^{v}(1 \leqq i \leqq g)$. Then

$$
\begin{aligned}
P_{\iota} A(x) & =\left(\sum_{v \geq 0} d_{\imath, v}(y) \partial_{z}^{v}\right)\left(\sum_{w \geq 0} A_{w}(y) z(w)\right) \\
& \equiv \sum_{v \geqq 0} d_{\imath, v}(y) A_{v}(y)=B_{\imath}(y) \quad\left(\bmod K m_{X, p}\right) .
\end{aligned}
$$

Set $L=\sum_{i-1}^{g} \mathcal{O}_{X}, p\left(P_{\imath} A(x)\right)$. By (5.6.4), $B_{\imath}(y) \cong L+K m_{X, p}$. Since $\left\{B_{1}(y), \cdots, B_{g}(y)\right\}$ generates the $\mathcal{O}_{X}, p$-module $K$,

$$
K \subset L+K m_{X, p} .
$$

On the other hand, by (5.6.3), $P_{\jmath} 4(x)=P_{\jmath}\left(y, \partial_{z}\right) \sum_{\imath=1}^{g} B_{\imath}(y) c_{\imath}(x)=\sum_{\imath=1}^{g} B_{\imath}(y) \cdot P_{\jmath} c_{\imath}(x)$ $\equiv K$. Hence

$$
L \subset K
$$

By (5.6.5), (5.6.6) and the "Nakayama's lemma", we get $K=L$. Hence there exist $e_{\imath j}(x) \in \mathcal{O}_{X, p}(1 \leqq i, j \leqq g)$ such that $B_{\imath}(y)=\sum_{j=1}^{g} e_{\imath \jmath}(x) \cdot P_{\jmath}\left(y, \partial_{z}\right) A(x)$. By 
setting $Q_{\imath}\left(x, \partial_{z}\right)=\sum_{j=1}^{g} e_{\imath \jmath}(x) P_{j}\left(y, \partial_{z}\right)$, we get the following lemma.

Lemma 5.7. Given $A(x)=\left(a_{1}(x), \cdots, a_{n}(x)\right)=\mathcal{O}_{X, p}^{n}$, there exist $n$-tuples $B_{2}(y)$ $=\left(b_{\imath 1}(y), \cdots, b_{\imath n}(y)\right) \in \mathcal{O}_{Y, f(p)}^{n}, Q_{\imath} \sqsubseteq \mathscr{D}_{f, p}$ and $c_{\imath}(x) \in \mathcal{O}_{X, p}(1 \leqq i \leqq g)$ such that $B_{\imath}(y)$ $=Q_{\imath} A(x)(1 \leqq i \leqq g)$, and $A(x)=\sum_{i=1}^{g} c_{\imath}(x) B_{\imath}(y)$.

5.8. Proof of (5.2). Let $u$ be a section of $M=f^{*} M^{\prime}=\mathcal{O}_{X} \otimes_{f-1 O_{Y}} M^{\prime}$ on an open neighbourhood $U$ of $p$. By shrinking $U$, if necessary, we may assume that $u$ can be expressed as $u=\sum_{j=1}^{n} a_{j}(x) \otimes u_{j}^{\prime}$ with $a_{j} \equiv \Gamma\left(U, \mathcal{O}_{x}\right)$ and $u_{j}^{\prime} \equiv$ $\Gamma\left(f(U), M^{\prime}\right)$. (Note that $f$ is an open mapping.) Let $A(x)=\left(a_{1}(x), \cdots, a_{n}(x)\right) E$ $\mathcal{O}_{X, p}^{n}$, and take $B_{\imath}(y)=\left(b_{\imath 1}(y), \cdots, b_{\imath n}(y)\right), Q_{\imath}$ and $c_{\imath}(x)$ as in (5.7). Set $v_{\imath}^{\prime}=$ $\sum_{j=1}^{n} b_{\imath \jmath}(y) u_{j}^{\prime}$. Then $Q_{\imath} u=\sum_{\jmath=1}^{n} Q_{\imath} a_{j}(x) \otimes u_{\jmath}^{\prime}=\sum_{j=1}^{n} b_{\imath j}(y) \otimes u_{j}^{\prime}=\sum_{j=1}^{n} 1 \otimes b_{\imath j}(y) u_{j}^{\prime}=$ $1 \otimes v_{i}^{\prime}$ and $\sum_{i=1}^{g} c_{\imath}(x)\left(1 \otimes v_{i}^{\prime}\right)=\sum_{i=1}^{g} \sum_{j=1}^{n} c_{\imath}(x) b_{i j}(y) \otimes u_{j}^{\prime}=\sum_{j=1}^{n} a_{j}(x) \otimes u_{j}^{\prime}=u$. If we set $\sum_{i=1}^{g} \mathcal{O}_{Y, f(p)} v_{\imath}^{\prime}=T(u, p)$, then $\mathscr{D}_{f, p} u=\sum_{\imath=1}^{g} \mathcal{O}_{Y, p}\left(1 \otimes v_{\imath}^{\prime}\right)=\mathcal{O}_{X, p} \otimes_{\mathcal{O}_{Y, f(p)}} T(u, p)$. Since $\mathcal{O}_{X, p}$ is faithfully flat over $\mathcal{O}_{Y, f(p)}$, the $\mathcal{O}_{Y, f(p)}$-submodule $T(u, p)$ of $M_{f(p)}^{\prime}$ is uniquely determined by $u$ (cf. [7, Chap. 1, 33 , Prop. 10]). By shrinking $U$, if necessary, we may assume that $c_{\imath}$ 's and all the coefficients of $Q_{\imath}$ 's are regular on $U$. By the same argument as above, we get $\mathscr{D}_{f, p^{\prime}} u=\mathcal{O}_{X, p^{\prime}} \otimes_{\mathcal{O}_{Y, f\left(p^{\prime}\right)}}$ $\left(\sum_{i=1}^{g} \mathcal{O}_{Y, f\left(p^{\prime}\right)} v_{i}^{\prime}\right)$ for any $p^{\prime} \in U$. In other words, if we define a subsheaf $T(u, U)$ of $M^{\prime} \mid f(U)$ by $T(u, U)=\sum_{i=1}^{g} \mathcal{O}_{f(U)} v_{i}^{\prime}$, then $\left(\mathscr{D}_{f} \mid U\right) u=(f \mid U)^{*} T(u, U)$. By shrinking $U$, we may assume that $U$ is an affine open subset of $X$. Since we are assuming $N$ to be a quasi-coherent $\mathcal{O}_{X}$-module, there exist sections $u_{\alpha} \in \Gamma(U, N)$ $(\alpha \in A)$ such that $N \mid U=\sum_{\alpha \in A} \mathcal{O}_{U} u_{\alpha}$. Define an $\mathcal{O}_{f(U)}$-submodule $N^{\prime}(U)$ of $M^{\prime} \mid f(U)$ by $N^{\prime}(U)=\sum_{\alpha \in A} T\left(u_{a}, U\right)$. Then $N \mid U=\sum_{a \in A}\left(\mathscr{D}_{f} ; U\right) u_{\alpha}=\sum_{\alpha \in A}(f \mid U)^{*} T\left(u_{\alpha}, U\right)=$

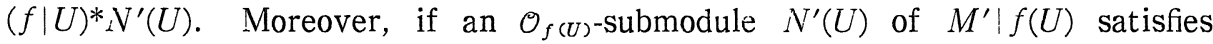

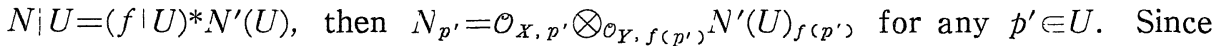
$\mathcal{O}_{X, p^{\prime}}$ is faithfully flat over $\mathcal{O}_{Y, f\left(p^{\prime}\right)}, N^{\prime}(U)_{f\left(p^{\prime}\right)}$ is uniquely determined by $N_{p^{\prime}}$. Hence $N^{\prime}(U)$ is unique. 'Thus we get the first assertion.

Let $p$ and $p^{\prime}$ be two points of $Y$. Take an open neighbourhood $U$ (resp.

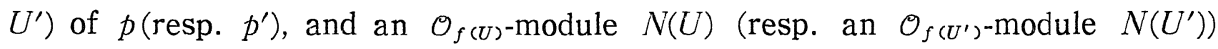
as in the first part. If $p^{\prime \prime} E U \cap U^{\prime}, N_{p^{\prime \prime}}=\mathcal{O}_{X, p^{\prime \prime}} \otimes_{\mathcal{O}_{Y}, f\left(p^{\prime \prime}\right)} N^{\prime}(U)_{f\left(p^{\prime \prime}\right)}=\mathcal{O}_{X, p^{\prime \prime}}$ $\otimes_{\mathcal{O}_{Y, f\left(p^{\prime \prime}\right)}} N^{\prime}\left(U^{\prime}\right)_{f\left(p^{\prime \prime}\right)}$. Hence $N^{\prime}(U)=N^{\prime}\left(U^{\prime}\right)$ on $f\left(U \cap U^{\prime}\right)$. In order to get the second assertion, it is enough to prove that $N^{\prime}(U)=N^{\prime}\left(U^{\prime}\right)$ on $f(U) \cap f\left(U^{\prime}\right)$, assuming the connectedness of the fibres of $f$. Hence it is enough to prove that $f\left(U \cap U^{\prime}\right)=f(U) \cap f\left(U^{\prime}\right)$. Let $q \in f(U) \cap f\left(U^{\prime}\right)$. Then $f^{-1}(q) \cap U \neq \phi$ and $f^{-1}(q) \cap U^{\prime} \neq \phi$. Since $f^{-1}(q)$ is connected, we can find an element $p^{\prime \prime} \in f^{-1}(q) \cap$ $U \cap U^{\prime}$. Then $q=f\left(p^{\prime \prime}\right) \in f\left(U \cap U^{\prime}\right)$. Thus we have completed the proof.

\section{$\S 6$. Character of $V(w, \lambda, \mathfrak{p})$}

6.0. In this section, first we determine the character of $V(w, \lambda, \mathfrak{p})$. Once the character formula is obtained, we can deduce several consequences from it. See (6.6) and (6.8). 
6.1. Let $\lambda_{d}$ be the lowest weight of a finite dimensional irreducible $P$-module, and

$$
\mathrm{t}_{I, \text { rad }}^{2}\left(\lambda_{d}\right)=\left\{\lambda_{c}=\mathrm{t}_{I}^{\check{I}} \mid\left\langle\lambda_{c}+\lambda_{d}-\rho, \alpha^{\ulcorner}\right\rangle \neq 0,1,2, \cdots \quad \text { for any } \alpha \in R_{+}\right\} .
$$

An easy calculation using fundamental weights shows that $\mathrm{f}_{I_{1}, \mathrm{rad}_{d}}\left(\lambda_{d}\right)$ is an open dense subset of $\mathrm{t}_{I}^{2}$. Let $\lambda_{c} c \mathrm{t}_{I}^{2}, \lambda=\lambda_{c}+\lambda_{d}$, and assume that $\lambda_{c}=\mathrm{t}_{I, \text { rad }}{ }^{2}\left(\lambda_{d}\right)$. Let us consider the simplicity of the $U(\mathrm{~g})$-module

$$
M_{A}(w) * \otimes_{A, \lambda_{c}} C=H_{q^{-1} Y(w)}^{c d(w)}\left(X, O_{Y}(\lambda)\right)
$$

for $w \boxminus\left(W / W_{I}\right)_{l}$, where $c d(w)=l\left(w_{S}\right)-l(w)=\operatorname{codim}_{X} X(w)=\operatorname{codim}_{Y} Y(w)$ and $\Theta_{X}(\lambda)$ denotes (abusively) the $\mathscr{D}_{X}(\lambda)$-module $q^{*} \mathcal{O}\left(w U_{-}, \lambda_{c}\right) \otimes \mathcal{O}_{X}\left(\lambda_{d}\right)$. (Cf. (2.9).) Note that the inclusion $q^{-1} Y(w) \rightarrow X$ is an affine morphism and hence, $H_{q^{-1}{ }^{-1}(w)}^{(w)}\left(\mathcal{O}_{X}(\lambda)\right)$ $=R \Gamma_{q^{-1 Y(w)}}\left(\mathcal{O}_{Y}(\lambda)\right)[c d(w)]$ and $H_{q^{-1} Y^{\prime}(w)}^{(w)}\left(X, \mathcal{O}_{X}(\lambda)\right)=R \Gamma_{q^{-1 Y(}(w)}\left(X, \mathcal{O}_{Y}(\lambda)\right)[c d(w)]$. (To see this, it suffices to show that the inclusion morphism $Y(w) \rightarrow Y$ is affine, whose proof we do not give here since a similar argument appears later in (7.2).) Since

$$
H_{q^{-1}(w)}^{c d(w)}\left(X, \mathcal{O}_{X}(\lambda)\right)=\Gamma\left(X, H_{q^{-1}(w)}^{c d(w)}\left(\mathcal{O}_{X}\left(\lambda_{c}\right)\right) \otimes \mathcal{O}_{X}\left(\lambda_{d}\right)\right),
$$

the simplicity of the $U(\mathrm{~g})$-module $M_{A}(w)^{*} \otimes_{A, \lambda_{C}} C$ is equivalent to the simplicity of the $\mathscr{D}_{X}(\lambda)$-module

$$
H_{q^{-1}(w)}^{c d(w)}\left(\mathcal{O}_{X}\left(\lambda_{c}\right)\right) \otimes \mathcal{O}_{X}\left(\lambda_{d}\right)=q^{*} H_{Y(w)}^{c d(w)}\left(\mathcal{O}_{Y}\left(\lambda_{c}\right)\right) \otimes \mathcal{O}_{X}\left(\lambda_{d}\right),
$$

which is equivalent to the simplicity of the $\mathscr{D}_{X}\left(\lambda_{c}\right)$-module $H_{q^{-1}(w)}^{c d(w)}\left(\Theta_{X}\left(\lambda_{c}\right)\right)=$ $q^{*} H_{Y(w)}^{c d(w)}\left(\mathcal{O}_{Y}\left(\lambda_{c}\right)\right)$. (Note that $\lambda-\rho$ is regular anti-dominant, and use (3.4).) Furthermore, by (5.4), the simplicity of $q^{*} H_{Y(\omega)}^{c d(w)}\left(\Theta_{Y}\left(\lambda_{c}\right)\right)$ is equivalent to that of the $\mathscr{D}_{Y}\left(\lambda_{c}\right)$-module $H_{Y(w)}^{c d}\left(\mathcal{O}_{Y}\left(\lambda_{c}\right)\right)$.

6.2. Let $\lambda_{c} \in \mathrm{t}_{I, r a d}^{\sim}\left(\lambda_{d}\right) \cap \mathrm{t}_{I, r a d}^{r}(0)$, and assume that $H_{q^{-1} Y(w)}^{c d(w)}\left(X, O_{X}(\lambda)\right)$ is not simple. By (6.1), $N:=H_{q^{-1}{ }^{-1}(w)}^{c(w)}\left(X, \mathcal{O}_{X}\left(\lambda_{c}\right)\right)=\Gamma\left(H_{q^{-1} Y_{(w)}(w)}^{c d}\left(\mathcal{O}_{Y}\left(\lambda_{c}\right)\right)\right)$ is not simple. (See (3.2) for $\Gamma$.) Let $L$ be a simple submodule of $N$. Put $M_{w}:=M\left(w\left(\lambda_{c}-\rho\right)\right.$ $-\rho, \mathfrak{b})$. Since $N \subset H_{X(w)}^{c d(w)}\left(X, \mathcal{O}_{X}\left(\lambda_{c}\right)\right)=M_{w}^{*}$ by $(3.8), L^{*}(\simeq L)$ is the simple quotient of $M_{w}$. (The injectivity of $N=H_{q^{-1}(w)}^{c d(w)} \rightarrow H_{X(w)}^{c d(w)}$ follows from the vanishing of $H_{X(w)-q^{-1} Y(w)}^{c d(w)}\left(X, \mathcal{O}_{X}(\lambda)\right)$, which can be proved by the usual 'dévissage'.) Let $[Q] \in J H(N / L) \subset J H\left(M_{w}^{*} / L\right)=J H\left(M_{w}\right) \backslash\{[L]\}$. By (3.6), $Q=V\left(w^{\prime}\left(\lambda_{c}-\rho\right)-\rho\right)$ with some $w^{\prime} \leqq w$. By (3.4), $\otimes Q$ is a composition factor of $\otimes N=H_{q^{-1} Y(w)}^{c d(w)}\left(\mathcal{\Theta}_{X}\left(\lambda_{c}\right)\right)$. Hence

$$
\operatorname{supp}(\otimes Q)=\bigcup_{w^{\prime \prime} \in W_{0}} \overline{X\left(w^{\prime \prime}\right)} \text { for some } W_{0} \subset\left\{w^{\prime \prime} \equiv\left(W / W_{I}\right)_{l} \mid w^{\prime \prime} \leqq w\right\} .
$$

(Consider the characteristic variety.) On the other hand $\otimes V\left(w^{\prime}\left(\lambda_{c}-\rho\right)-\rho\right)^{*}$ is the simple submodule of $\otimes M_{w^{\prime}}^{*}=H_{X\left(w^{\prime}\right)}^{c d\left(w^{\prime}\right)}\left(\mathcal{O}_{X}\left(\lambda_{c}\right)\right)$. Since $H_{X\left(w^{\prime}\right)}^{c d\left(w^{\prime}\right)}\left(\mathcal{O}_{X}\left(\lambda_{c}\right)\right)$ does not

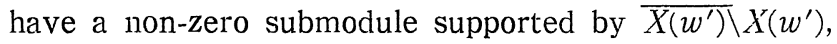

$$
\operatorname{supp}(\otimes Q)=\operatorname{supp}\left(\otimes Q^{*}\right)=\operatorname{supp}\left(\otimes V\left(w^{\prime}\left(\lambda_{c}-\rho\right)-\rho\right)^{*}\right)=\overline{X\left(w^{\prime}\right)} .
$$


By (6.2.1) and (6.2.2), $w^{\prime} \equiv\left(W / W_{I}\right)_{l}$. Since the highest weight $w^{\prime}\left(\lambda_{c}-\rho\right)-\rho$ of $Q$ is also a weight of $M_{w}=M\left(w\left(\lambda_{c}-\rho\right)-\rho, \mathfrak{b}\right)$,

$$
\left(w\left(\lambda_{c}-\rho\right)-\rho\right)-\left(w^{\prime}\left(\lambda_{c}-\rho\right)-\rho\right) \in Q_{+}, \quad w, w^{\prime} \Subset\left(W / W_{I}\right)_{l}, \quad \text { and } \quad w>w^{\prime} .
$$

Theorem 6.3. Let $\lambda_{d}$ be the lowest weight of a finite dimensional irreducible $P$-module, $\lambda_{c} \sqsubseteq \mathrm{f}_{I}^{2}, \lambda=\lambda_{c}+\lambda_{d}$, and $w \subseteq\left(W / W_{I}\right)_{l}$. Then

$$
\operatorname{ch} V(w, \lambda, \mathfrak{p})=e^{-w \rho-\rho} w\left(\operatorname{ch} V_{I}(\lambda)\right) \prod_{a \in R_{+} w R_{I}}\left(1-e^{-\alpha}\right)^{-1} .
$$

$\left(V_{I}(\lambda)\right.$ is the simple p-module with the lowest weight $\left.\lambda.\right)$

Proof. We calculate the character of $V(w, \lambda, \mathfrak{p})$ using (1.12). Since we have already proved that $\left(M_{A}(w)^{*}, \varphi\right)$ defined in $\S 4$ satisfies $\left(1.11 .1^{\prime}\right)$ and $\left(1.11 .2^{\prime}\right)$, it remains only to prove $\left(1.11 .3^{\prime}\right)$. (Cf. (4.1).) Let

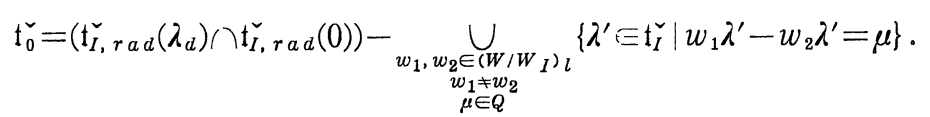

Then (6.2.3) does not hold for any $\lambda_{c}^{\prime} \boxminus \mathrm{t}_{0}^{\llcorner}$. Hence $M_{\boldsymbol{A}}(w)^{*} \otimes_{\boldsymbol{A}, \lambda_{c}^{\prime}} \boldsymbol{C}=H_{q^{-1}(w)(w)}^{c d(X)}(X$, $\left.\mathcal{O}_{X}\left(\lambda_{c}^{\prime}+\lambda_{d}\right)\right)$ is simple for $\lambda_{c}^{\prime} \cong \mathrm{t}_{0}^{\nu}$. Moreover $\mathrm{f}_{0}^{\sim}$ is an open dense subset of $\mathrm{t}_{\check{I}}$ with respect to the classical topology. Thus $M_{A}(w)^{*}$ satisfies $\left(1.11 .3^{\prime}\right)$. Hence

$$
\begin{aligned}
\operatorname{ch} V(w, \lambda, \mathfrak{p}) & =\operatorname{ch} M_{A}(w)^{*} \otimes_{A, \lambda_{c}} C & & \text { by }(1.12) \\
& =\operatorname{ch} M_{A}(w) \otimes_{A, \lambda_{c}} C & & \text { by (1.1) } \\
& =e^{-w \rho-\rho} \cdot w\left(\operatorname{ch} V_{I}(\lambda)\right) \prod_{a \in R_{+} \backslash w R_{I}}\left(1-e^{-\alpha}\right)^{-1} & & \text { by (4.8.2). }
\end{aligned}
$$

Corollary 6.4. Let $w \equiv\left(W / W_{I}\right)_{l}, \lambda_{d}$ be as in (6.3), and $\mu \in Q_{+}$.

(1) $V_{A}\left(w, c+\lambda_{d}, \mathfrak{p}\right)(\mu)$ is a free A-module of finite type.

(2) $\operatorname{ch} V_{K}\left(w, \eta+\lambda_{d}, \mathfrak{p}\right)=\operatorname{ch} M_{A}(w) * \otimes_{A} K=e^{-w \rho-\rho} \cdot w\left(e^{\eta} \operatorname{ch} V_{I}\left(\lambda_{d}\right)\right) \prod_{\alpha \in R_{+} \backslash w R_{I}}(1-$ $\left.e^{-a}\right)^{-1}$. (See $(4.1)$ for $V_{A}\left(w, c+\lambda_{d}, \mathfrak{p}\right)$ etc.)

Proof. Since $V_{A}\left(w, c+\lambda_{d}, \mathfrak{p}\right)(\mu)$ is a quotient of the finitely generated Amodule $M_{A}\left(w\left(c+\lambda_{d}-\rho\right)-\rho\right)(\mu)$, (cf. (1.17) and (4.1)) it is enough to prove that $V_{A}\left(w, c+\lambda_{d}, \mathfrak{p}\right)(\mu)$ is a projective $A$-module [26], [32]. The projectivity follows from the following lemma together with (6.3).

Lemma 6.5. Let $C$ be a polynomial ring over a field and $M$ a $C$-module of finite type. Assume that the dimension of the $(C / m)$-vector space $M / m M$ does not depend on the maximal ideal $m$ of $C$. Then $M$ is a projective $C$-module.

Proof. It is enough to prove that the quasi-coherent sheaf $\tilde{M}$ on $\operatorname{Spec} C$ corresponding to $M$ is $\widetilde{C}$-free in a neighbourhood of any closed point $m \in \operatorname{Spec} C$, where $\tilde{C}$ is the structure sheaf. Let $C_{m}$ be the local ring at $m$, and let 
$u_{1}, \cdots, u_{n}$ be elements of $M$ which give a basis of $M / m M$. Let $e_{1}, \cdots, e_{n}$ be the natural basis of $C^{n}$ and define a $C$-homomorphism $\varphi: C^{n} \rightarrow M$ by $\varphi\left(e_{\imath}\right)=u_{\imath}$. Let $\left\{v_{j}\right\}$ be a generator system of $M$. Since $\varphi \otimes_{C} C_{m}$ is surjective by the "Nakayama's lemma", $v_{\jmath}=\sum_{\imath} u_{\imath} c_{\imath \jmath}$ with some $c_{\imath j} \subseteq C_{m}$. Take an element $f \equiv C$ such that all the $c_{\imath \jmath}$ 's come from $C_{f}=C\left[f^{-1}\right]$. Then $\varphi \otimes C_{f}$ is surjective. Let $W_{f}=i, 1 \otimes_{C} C_{f}$ and $K=\operatorname{ker} \varphi \otimes C_{f}$. From the exact sequence $0 \rightarrow K \rightarrow C_{f}^{n} \rightarrow M_{f} \rightarrow 0$, we get the exact sequence $\left(C / m^{\prime}\right) \otimes K \rightarrow\left(C / m^{\prime}\right)^{n} \rightarrow M / m^{\prime} M \rightarrow 0$ for any maximal ideal $m^{\prime}$ contained in $\operatorname{Spec} C_{f}^{\prime}$. Since the dimension of the $\left(C / m^{\prime}\right)$-vector space $M / m^{\prime} M$ is equal to $n$, the image of $\left(C / m^{\prime}\right) \otimes K$ in $\left(C / m^{\prime}\right)^{n}$ equals 0 , i. e., $K \subset$ $m^{\prime} C_{f}^{n}$. Since the intersection of $m^{\prime} C_{f}^{n}$ for maximal ideals $m^{\prime}$ of $C_{f}$ is 0 , we get $K=0$ and $M_{f}=C_{f}^{n}$.

Theorem 6.6. Let $\lambda_{c}, \lambda_{d}, \lambda$ and $w$ be as in (6.3). If $\lambda-\rho$ is anti-dominant, then

$$
\Gamma\left(X, q^{*} H_{Y(w)}^{c d(w)}\left(\mathcal{O}_{Y}\left(\lambda_{c}\right)\right) \otimes \mathcal{O}_{X}\left(\lambda_{d}\right)\right)=H_{q^{-1} Y^{\prime}(w)}^{c d(w)}\left(X, \mathcal{O}_{X}(\lambda)\right) \cong V(w, \lambda, \mathfrak{p})^{*}
$$

as $U(\mathrm{~g})$-modules. (See (6.1) for $\mathcal{O}_{X}(\lambda)$.)

Proof. Using the notation of (4.1), put $M_{A}=M_{A}\left(w\left(c+\lambda_{d}-\rho\right)-\rho\right), J_{A}$ $=J_{A}\left(w, c+\lambda_{d}, \mathfrak{p}\right), V_{A}=M_{A} / J_{A}, M_{K}=M_{A} \otimes_{A} K, J_{K}=J_{A} \otimes_{A} K, V_{K}=V_{A} \otimes_{A} K$, and $i_{K}(w)^{*}=M_{A}(w) * \otimes_{A} K$. First, let us show that the kernel of the homomorphism $\varphi: M_{A}\left(w\left(c+\lambda_{d}-\rho\right)-\rho\right) \rightarrow M_{A}(w)^{*}$ defined in (4.7) and (4.8) is $J_{A}$. Consider the diagram

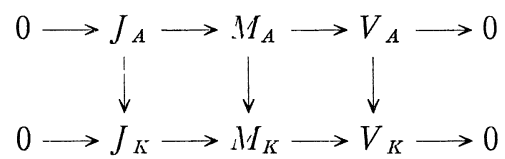

consisting of the natural morphisms. Since $V_{A}$ is a free A-module by $(6.4,(1))$, the first horizontal sequence is a split exact sequence, and hence the second one is also exact. Since $M_{A}$ and $V_{A}$ are 1 -free, the second and the third vertical arrows are injections, and hence the remaining one is also an injection. (In fact, we can show that $J_{A}$ is a free $A$-module together with its 'weight spaces' using [26], [32] and (1.17.2).) By (1.4, (2)), $V_{K}$ is the simple quotient of the Verma module $M_{K}$ and $J_{K}$ the maximal submodule of $M_{K}$. Since $\operatorname{ch} V_{K}=$ $\operatorname{ch} M_{K}(w)^{*}$ by $(6.4,(2)), V_{K} \cong M_{K}(w)^{*}$. Since $M_{A}(w)^{*}$ is $A$-free, the natural morphism $M_{A}(w)^{*} \rightarrow M_{K}(w)^{*}$ is injective, $\varphi_{K}:=\varphi \otimes K: M_{K} \rightarrow M_{K}(w)^{*}$ is not identically zero, and hence surjective. Thus we can identify $\varphi_{K}: M_{K} \rightarrow M_{K}(w)^{*}$ with the projection of the Verma module to its simple quotient. Especially its kernel is $J_{K}$. Hence $J_{A} \subset M_{A} \cap J_{K}=M_{A} \cap \operatorname{ker} \varphi_{K}=\operatorname{ker} \varphi$. Since $\operatorname{ker} \varphi$ is contained in $M_{A}\left(w\left(c+\lambda_{d}-\rho\right)-\rho\right)_{+}, \operatorname{ker} \varphi=J_{A}$. Thus $\varphi$ induces homomorphisms $V_{A}(w, c+$ $\left.\lambda_{d}, \mathfrak{p}\right) \rightarrow M_{A}(w)^{*}, V(w, \lambda, \mathfrak{p}) \rightarrow M_{A}(w)^{*} \otimes_{A, \lambda_{c}} C=H_{q^{-1} Y(w)}^{c d(w)}\left(X, \mathcal{O}_{X}(\lambda)\right)^{*}$ and $\sigma^{*}:$ $H_{q^{-1}(w)}^{c d(w)}\left(X, \mathcal{O}_{X}(\lambda)\right) \rightarrow V\left(w^{\prime}, \lambda, \mathfrak{p}\right)^{*}$. Note that the image of $\sigma^{*}$ contains the weight 
space of the highest weight. Let $K=\operatorname{ker} \sigma^{*}$. By (3.8), $M(w(\lambda-\rho)-\rho, \mathfrak{h})^{*}=$ $H_{X(w)}^{c d(w)}\left(X, \mathcal{O}_{X}(\lambda)\right) \supset H_{q^{-1}(w)}^{c(w)}\left(X, \mathcal{O}_{X}(\lambda)\right) \supset K$, and $K^{*}$ is a quotient of $M(w(\lambda-\rho)-\rho$, b). If $K \neq 0$, then $K$ contains the weight space of weight $w(\lambda-\rho)-\rho$. This contradicts the fact that the image of $\sigma^{*}$ contains the weight space of weight $w(\lambda-\rho)-\rho$. Hence $K=0$ and $\sigma^{*}$ is injective. Since

$$
\begin{aligned}
\operatorname{ch} H_{q^{-1} Y(w)}^{c \alpha(w)}\left(X, \mathcal{O}_{X}(\lambda)\right) & =\operatorname{ch} M_{A}(w) \otimes_{A, \lambda_{c}} C & & (\operatorname{cf} .(4.8)) \\
& =e^{-w o-\rho} \cdot w\left(\operatorname{ch} V_{I}(\lambda)\right) \prod_{\alpha \in R_{+} \backslash w R_{I}}\left(1-e^{-a}\right)^{-1} & & \text { by }(4.8 .2) \\
& =\operatorname{ch} V(w, \lambda, \mathfrak{p})^{*} & & \text { by (6.3), }
\end{aligned}
$$

$\sigma^{*}$ is an isomorphism.

Remark 6.6.1. In the above theorem, we can not omit the assumption that $\lambda-\rho$ is anti-dominant. See (3.9) and (1.19).

6.7. Generalized Verma modules. Let $V_{I}^{\prime}\left(\lambda^{\prime}\right)$ be the finite dimensional irreducible $\mathfrak{p}(I)$-module with the highest weight $\lambda^{\prime}$, and

$$
M\left(\lambda^{\prime}, \mathfrak{p}\right)=U(\mathfrak{g}) \otimes_{U(\mathfrak{p})} V_{I}^{\prime}\left(\lambda^{\prime}\right) .
$$

Such a $U(\mathrm{~g})$-module is called a generalized Verma module. Obviously

$$
\operatorname{ch} M\left(\lambda^{\prime}, \mathfrak{p}\right)=\operatorname{ch} V_{I}^{\prime}\left(\lambda^{\prime}\right) \prod_{\alpha \in R_{+} \backslash R_{I}}\left(1-e^{-a}\right)^{-1} .
$$

Theorem 6.8. Let $I \subset S, w \in\left(W / W_{I}\right)_{l}$ and $\lambda$ be a character of $\mathrm{t}$ such that $\left\langle\lambda, \alpha^{`}\right\rangle\left(\alpha \in \Pi_{I}\right)$ are non-positive integers. If

$$
J:=w I w^{-1} \subset S,
$$

then $w(\lambda-\rho)-\rho$ is the highest weight of a finite dimensional irreducible $\mathfrak{p}(J)$ module, and

$$
M(w(\lambda-\rho)-\rho, \mathfrak{p}(J))=V(w, \lambda, \mathfrak{p}(I)) .
$$

Proof. Since $w \in\left(W / W_{I}\right)_{l}, w\left(\Pi_{I}\right)=-\Pi_{J}$. Let $\alpha \in \Pi_{I}$ and $w(\alpha)=-\beta$. Then $\beta \equiv \Pi_{J}$ and $\left\langle w(\lambda-\rho)-\rho, \beta^{\sim}\right\rangle=\left\langle\lambda, w^{-1} \beta^{\sim}\right\rangle-\left\langle\rho, w^{-1} \beta^{\sim}\right\rangle-\left\langle\rho, \beta^{\sim}\right\rangle=-\left\langle\lambda, \alpha^{\sim}\right\rangle+\langle\rho$, $\left.\alpha^{\nu}\right\rangle-\left\langle\rho, \beta^{\nu}\right\rangle=-\left\langle\lambda, \alpha^{\nu}\right\rangle$. (Note that $\left\langle\rho, \gamma^{\nu}\right\rangle=1$ for $\gamma \in \Pi$.) Thus $\langle w(\lambda-\rho)-\rho$, $\left.\beta^{\sim}\right\rangle\left(\beta \in \Pi_{J}\right)$ are non-negative integers, and we can consider the generalized Verma module $M(w(\lambda-\rho)-\rho, \mathfrak{p}(J))$. Consider the $U_{A}(\mathfrak{b})$-module $A(w(c-\rho)-\rho)$ as in (1.3). Extend this $U_{A}(\mathfrak{b})$-module to a $U_{A}(\mathfrak{p}(J))$-module by putting $\mathrm{g}(\alpha) 1_{w(c-\rho)-\rho}=0$ for $\alpha \subseteq R_{-} \cap R_{J}$. (See (1.3) for $1_{w(c-\rho)-\rho}$. Note that the $W_{I^{-}}$ invariance of $c$ implies the $W_{J}$-invariance of $w(c-\rho)-\rho$.) Let $w V_{I}\left(\lambda_{d}\right)$ be the irreducible $P(J)$-module with the highest weight $w \lambda_{d}$. Then $A(w(c-\rho)-\rho)$ $\otimes_{c} w V_{I}\left(\lambda_{d}\right)$ has a $U_{A}(\mathfrak{p}(J))$-module structure. Let

$$
M_{A}\left(w\left(c+\lambda_{d}-\rho\right)-\rho, \mathfrak{p}(J)\right):=U_{A}(g) \otimes_{\left.U_{A}^{(p)}(J)\right)}\left(A(w(c-\rho)-\rho) \otimes w V_{I}\left(\lambda_{d}\right)\right) .
$$


It is enough to prove that

$$
M_{A}\left(w\left(c+\lambda_{d}-\rho\right)-\rho, \mathfrak{p}(J)\right) \cong V_{A}\left(w, c+\lambda_{d}, \mathfrak{p}(I)\right) .
$$

Denote the left (resp. right) hand side of (6.8.2) by $M_{A}$ (resp. $V_{A}$ ). As is easily seen, $M_{A}$ is isomorphic to $U_{A}\left(\mathfrak{u}_{-}(J)\right) \otimes_{C} w V_{I}\left(\lambda_{d}\right)$ as an $A$-module, and the kernel of the natural homomorphism $M_{A}\left(w\left(c+\lambda_{d}-\rho\right)-\rho, \mathfrak{b}\right) \rightarrow M_{A}\left(w\left(c+\lambda_{d}-\rho\right)-\right.$ $\rho, \mathfrak{p}(J))$ is contained in $M_{A}\left(w\left(c+\lambda_{d}-\rho\right)-\rho, \mathfrak{b}\right)_{+}$. Hence we get a surjective $U_{A}(\mathrm{~g})$-homomorphism $\psi: M_{A} \rightarrow V_{A}$. For $\mu \in Q_{+}$, let $M_{A}(\mu)$ and $V_{A}(\mu)$ be the images of $M_{A}\left(w\left(c+\lambda_{d}-\rho\right)-\rho, \mathfrak{b}\right)(\mu)$ (i. e., 'the weight space' of weight $w\left(c+\lambda_{d}\right.$ $-\rho)-\rho-\mu)$ by the natural projections. As is easily seen, $M_{A}$ is a direct sum of $M_{A}(\mu)$ 's. On the other hand, $V_{A}$ is also a direct sum of $V_{A}(\mu)$ 's, by (1.17.3). Since $\phi$ induces a surjective $A$-homomorphism $\phi(\mu): M_{A}(\mu) \rightarrow V_{A}(\mu)$ for each $\mu$, it is enough to show that $\phi(\mu)$ is bijective. As is easily seen, $M_{A}(\mu)$ is a free $A$-module of finite type. On the other hand, $V_{A}(\mu)$ is also a free $A$-module of finite type by $(6.4,(1))$. Since $\psi(\mu)$ is a surjection between free $A$-modules of finite type, it is enough to show that $\operatorname{dim} M_{A}(\mu) \otimes_{A, \lambda_{c}} C=\operatorname{dim} V_{A}(\mu) \otimes_{A, \lambda_{c}} C$ for $\lambda_{c} \in \mathrm{t}_{I}^{\check{y}}$, i. e.,

$$
\operatorname{ch} M(w(\lambda-\rho)-\rho, \mathfrak{p}(J))=\operatorname{ch} V(w, \lambda, \mathfrak{p}(I)),
$$

where $\lambda=\lambda_{c}+\lambda_{d}$. By (6.7.1) and the Weyl's character formula, the left hand side of (6.8.3) is equal to

$$
\sum_{w^{\prime \prime} \in W_{J}} \varepsilon\left(w^{\prime \prime}\right) e^{w^{\prime \prime}(w(\lambda-\rho)-\rho+\rho(J))-\rho(J)} \prod_{\alpha \in R_{+}}\left(1-e^{-\alpha}\right)^{-1},
$$

where $\varepsilon\left(w^{\prime \prime}\right)=(-1)^{l\left(w^{\prime \prime}\right)}$ and $\rho(J)=(1 / 2) \sum_{\alpha \in R_{+} \cap R_{J}} \alpha$. Let $\beta \equiv \Pi_{J}$ and $\alpha=-w^{-1}(\beta)$ $\left(\subseteq \Pi_{I}\right)$. Then $\left\langle w \rho+\rho, \beta^{\sim}\right\rangle=-\left\langle\rho, \alpha^{\nu}\right\rangle+\left\langle\rho, \beta^{\sim}\right\rangle=0$, and hence $w \rho+\rho$ is $W_{J^{-}}$ invariant. Since $w W_{I} w^{-1}=W_{J}$ and $w \rho(I)=-\rho(J),(6.8 .4)$ is equal to

$$
e^{-w \rho-\rho} \sum_{w^{\prime} \in W_{I}} \varepsilon\left(w^{\prime}\right) e^{w w^{\prime}(\lambda-\rho(I))+w \rho(I)} \prod_{\alpha \in R_{+}}\left(1-e^{-a}\right)^{-1} .
$$

On the other hand, by (6.3) and the Weyl's character formula again, the right hand side of (6.8.3) is equal to

$$
\begin{aligned}
& e^{-w \rho-\rho} w\left(\sum_{w^{\prime} \in W_{I}} \varepsilon\left(w^{\prime}\right) e^{w^{\prime}\left(w_{I} \lambda+\rho(I)\right)-\rho(I)} \prod_{\alpha \in R_{+} \cap R_{I}}\left(1-e^{-a}\right)^{-1}\right) \\
& \cdot \prod_{a \in R_{+} \backslash w R_{I}}\left(1-e^{-a}\right)^{-1} .
\end{aligned}
$$

(Note that the highest weight of $V_{I}(\lambda)$ is $w_{I} \lambda$.) Since

$$
\begin{aligned}
& w \prod_{\alpha \in R_{+} \cap R_{I}}\left(1-e^{-a}\right)^{-1}=w\left(\varepsilon\left(w_{I}\right) e^{2 \rho(I)} \prod_{\alpha \in R_{+} \cap R_{I}}\left(1-e^{\alpha}\right)^{-1}\right) \\
& \quad=\varepsilon\left(w_{I}\right) e^{2 w \rho(I)} \prod_{\alpha \in R_{+} \cap R_{J}}\left(1-e^{-\alpha}\right)^{-1},
\end{aligned}
$$

replacing $w^{\prime}$ with $w^{\prime} w_{I}$ in (6.8.6), we can see that (6.8.5) is equal to (6.8.6). Thus we get (6.8.3) and complete the proof. 
Remark 6.9. Let us show that the set

$$
\left\{V(w, \lambda, \mathfrak{p}(I)) \mid I \subset S, w \in\left(W / W_{I}\right)_{l},\left\langle\lambda, \alpha^{\sim}\right\rangle \cong Z_{\leqq 0} \text { for } \alpha \subseteq \Pi_{I}\right\} / \cong
$$

is strictly larger than the set

$$
\left\{M\left(\lambda^{\prime}, \mathfrak{p}(J)\right) \mid J \subset S,\left\langle\lambda^{\prime}, \beta^{\sim}\right\rangle \in \boldsymbol{Z}_{\geq 0} \text { for } \beta \in \Pi_{J}\right\} / \cong .
$$

Let $M\left(\lambda^{\prime}, \mathfrak{p}(J)\right)$ be a module which belongs to (6.9.2). Then by (6.8), $M\left(\lambda^{\prime}, \mathfrak{p}(J)\right)$ $=V\left(w_{S}, w_{s} \lambda^{\prime}, \mathfrak{p}\left(w_{S} J w_{s}\right)\right)$ belongs to (6.9.1). Next let us find a module which belongs to (6.9.1) but not to (6.9.2). For this purpose, it suffices to show that $V(w, \lambda, \mathfrak{p}(I))$ in (6.9.1) with $\lambda \in \mathrm{f}_{I}^{2}$ belongs to (6.9.2) if and only if $w\left(\Pi_{I}\right) \subset-\Pi$. Assume that $V(w, \lambda, \mathfrak{p}(I))$ is isomorphic to some module $M\left(\lambda^{\prime}, \mathfrak{p}(J)\right)$ in the set (6.9.2). By the Weyl's character formula and by (6.7.1),

$$
\operatorname{ch} M\left(\lambda^{\prime}, \mathfrak{p}(J)\right)=\sum_{w^{\prime} \in W_{J}} \varepsilon\left(w^{\prime}\right) e^{w^{\prime}\left(\lambda^{\prime}+\rho(J)\right)-\rho(J)} \prod_{\alpha \in \boldsymbol{R}_{+}}\left(1-e^{-a}\right)^{-1} .
$$

Note that the numerator of the right hand side of (6.9.3) can be expressed as $e^{\lambda^{\prime}} \cdot f$, where $f$ is a Laurent polynomial in $\left\{e^{\beta} \mid \beta \subseteq \Pi_{J}\right\}$. Hence among the factors of the denominator, $\left(1-e^{-\alpha}\right)$ for $\alpha \in R_{+} \backslash R_{J}$ can not be canceled. (Note that the group ring of the root lattice is a unique factorization domain.) On the other hand, for $\lambda \equiv \mathrm{t}_{I}^{2}$,

$$
\operatorname{ch} V(w, \lambda, \mathfrak{p}(I))=e^{w(\lambda-\rho)-\rho} \prod_{\alpha \in R_{+} \backslash w R_{I}}\left(1-e^{-\alpha}\right)^{-1},
$$

by (6.3). Hence

$$
R_{+} \backslash R_{J} \subset R_{+} \backslash w R_{I} .
$$

By (6.9.3) and (6.9.4), we also get

$$
e^{w(\lambda-\rho)-\rho+\rho(J)} \prod_{\alpha \in R_{+} \cap w R_{I}}\left(1-e^{-\alpha}\right)=\sum_{w^{\prime} \in W_{J}} \varepsilon\left(w^{\prime}\right) e^{w^{\prime}\left(\lambda^{\prime}+\rho(J)\right)} .
$$

Since the right hand side is $W_{J}$-antisymmetric, the left hand side is divisible by $\Pi_{\alpha \in R_{+} \cap R_{J}}\left(1-e^{-\alpha}\right)[6$, Chap. 6, no. 3.3, Prop. 2]. Thus (6.9.5) becomes an equality. Since $w \in\left(W / W_{I}\right)_{l}, w\left(R_{I} \cap R_{+}\right)$should be equal to $-\left(R_{J} \cap R_{+}\right)$, and

$$
w\left(\Pi_{I}\right)=-\Pi_{J} .
$$

(Moreover, comparing the highest terms of (6.9.6), we get

$$
\left.w(\lambda-\rho)-\rho=\lambda^{\prime}\right) .
$$

Conversely, assume $V(w, \lambda, \mathfrak{p}(I))$ in (6.9.1) is given and $w\left(\Pi_{I}\right) \subset-\Pi$. Then, by (6.8), $V(w, \lambda, \mathfrak{p}(I))=M\left(w(\lambda-\rho)-\rho, \mathfrak{p}\left(w I w^{-1}\right)\right)$ belongs to (6.9.2). Hence, if $\lambda$ is $W_{I}$-invariant, $V(w, \lambda, \mathfrak{p}(I))$ in (6.9.1) belongs to (6.9.2) if and only if $w\left(\Pi_{I}\right) \subset-\Pi$. Thus (6.9.1) is strictly larger than (6.9.2). In other words, our $U(\mathfrak{g})$-module $V(w, \lambda, \mathfrak{p})$ is a further generalization of generalized Verma modules. 


\section{$\S 7$. Resolutions of $V(w, \lambda, \mathfrak{p})$}

7.0. Resolutions of a finite dimensional $U(\mathrm{~g})$-modules by the Verma modules were constructed in [2] and [22] in several ways. In this section, we shall construct resolutions of $V(w, \lambda, \mathfrak{p})$ by the Verma modules. First we construct a resolution of $V(w, \lambda, \mathfrak{p})$ using the Grothendieck-Cousin complex [22]. Then using this resolution instead of $[2,9.9]$, and following the argument of [2], we construct a second resolution, which is a generalization of the resolution of a finite dimensional representation constructed by Bernstein-Gelfand-Gelfand $\left[2,10.1\right.$ and $\left.10.1^{\prime}\right]$. In order to reduce our task, we consider only the case where $\lambda$ is $W_{I}$-invariant.

7.1. Grothendieck-Cousin complex. We fix an element $w: \equiv\left(W / W_{I}\right)_{l}$ until the end of (7.6). Let $W_{I}^{(i)}=\left\{x \subseteq W_{I} \mid l(x)=i\right\}, W(w, i)=\left\{w^{\prime} \leqq W \mid w \geqq w^{\prime}, l\left(w^{\prime}\right)=\right.$ $l(w)-i\}, Z_{\imath}=\cup_{j \geqq \imath} \cup_{x \in W(w, j)} X(x)$ for $i \geqq 0, Z_{i}^{\prime}=Z_{i}$ for $i>0$ and $Z_{i}^{\prime}=X$ for $i \leqq 0$. (See (3.7) and (4.2) for $X(w)$ etc. Note that $W(w, i) \cap w W_{I}=w W_{I}^{(\imath)}$ and $\left(Z_{\imath}^{\prime}-\right.$ $\left.Z_{i+1}^{\prime}\right) \cap q^{-1} Y(w)=\cup_{x \in W_{I}^{(i)}} X(w x)$.) For any sheaf $\mathscr{F}^{\prime}$ on $X, X=Z_{0}^{\prime} \supset Z_{1}^{\prime} \supset \cdots \supset Z_{l^{\prime}}^{\prime} \supset$ $Z_{l^{\prime}+1}^{\prime}=\phi$ gives the global Cousin complex of $\mathscr{F}^{\prime}$ with respect to the filtration $\left\{Z_{\imath}^{\prime}\right\}$

$$
0 \longrightarrow \Gamma\left(X, \mathscr{I}^{\prime}\right) \longrightarrow H_{Z_{0}^{\prime} / Z_{1}^{\prime}}^{0}\left(X, \mathscr{I}^{\prime}\right) \longrightarrow H_{Z_{1}^{\prime} / Z_{2}^{\prime}}^{1}\left(X, \mathscr{I}^{\prime}\right) \longrightarrow \cdots .
$$

See $[22,7.8]$. We have also the local Cousin complex $[22,8.6]$

$$
0 \longrightarrow \mathscr{F}^{\prime} \longrightarrow H_{Z_{0}^{\prime} / Z_{1}^{\prime}}^{0}\left(\mathscr{I}^{\prime}\right) \longrightarrow H_{Z_{1}^{\prime} / Z_{2}^{\prime}}^{1}\left(\mathscr{F}^{\prime}\right) \longrightarrow \cdots .
$$

7.2. Let $\mathcal{C}$ be the full subcategory of the category of quasi-coherent $\mathcal{O}$ modules consisting of sheaves whose supports are contained in $\overline{q^{-1} Y(w)}$. Then $\mathcal{C}$ is closed under kernels, cokernels and extensions. Since $H_{X / Z_{i}^{\prime}}^{j}\left(\mathscr{F}^{\prime}\right) \in \mathcal{C}$ for any $\mathscr{F}^{\prime} \equiv \mathcal{C}$ and $i, j \equiv \boldsymbol{Z}$, all the basic assumptions of [22, 9.4 and several lines preceding it] are satisfied with our category $\mathcal{C}$ and the filtration $\left\{Z_{\imath}^{\prime}\right\}$. Let us show that the following conditions are satisfied for $i \geqq 0$.

$(L . V .)_{2} \quad R \Gamma_{X / Z_{i+1}^{\prime}}\left(\mathscr{F}^{\prime}\right)=\Gamma_{X / Z_{i+1}^{\prime}}\left(\mathscr{F}^{\prime}\right)$ if $\mathscr{F}^{\prime} \subseteq \mathcal{C}$ and $\operatorname{supp} \mathscr{F}^{\prime} \subset Z_{i}^{\prime}$.

Let $\iota_{i}^{\prime}: Z_{i}^{\prime}-Z_{{ }_{1+1}}^{\prime} \rightarrow X$ be the inclusion mappings. If $i>0$, then the connected components of $Z_{i}^{\prime}-Z_{i+1}^{\prime}$ are affine spaces $X(x)(x \in W(w, i))$, and hence for any $w^{\prime} \in W, \iota_{i}^{\prime-1}\left(w^{\prime} X\left(w_{S}\right)\right)$ is a disjoint union of $X(x) \cap w^{\prime} X\left(w_{S}\right)(x \equiv W(w, i))$. Each $X(x) \cap w^{\prime} X\left(w_{S}\right)$ is empty or a complement of a hypersurface in the affine space $X(x)$, and hence $\iota_{i}^{\prime-1}\left(w^{\prime} X\left(w_{S}\right)\right)(i>0)$ are affine varieties. Since $\left\{w^{\prime} X\left(w_{S}\right) \mid w^{\prime} \in W\right\}$ is an affine open covering of $X, c_{\imath}^{\prime}(i>0)$ are affine morphisms. Hence by the argument of the proof of $[22,9.6]$, we can prove $(L . V .)_{2}$ for $i>0$. Since $R \Gamma_{X / Z_{1}^{\prime}}\left(\mathscr{F}^{\prime}\right)=R \Gamma_{Z_{0} / Z_{1}}\left(\mathscr{F}^{\prime}\right)$ and $\Gamma_{X / Z_{1}^{\prime}}\left(\mathscr{F}^{\prime}\right)=\Gamma_{Z_{0} / Z_{1}}\left(\mathscr{F}^{\prime}\right)$ for $\mathscr{F}^{\prime} \mathcal{E}_{=} \mathcal{C}$, we get $(L . V .)_{0}$ by the same argument. Thus the condition (L.V.) of $[22, \mathrm{p} 362]$ is satisfied. In a similar way, using the fact that $Z_{i}-Z_{\imath+1}$ are affine varieties, we can show 
that the condition (G.V.) of $[22, \mathrm{p} 362]$ is also satisfied.

7.3. First construction of a resolution. Let $m:=c d(w)=\operatorname{codim}_{Y} Y(w)=$ $\operatorname{codim}_{X} q^{-1} Y(w)=l\left(w_{S}\right)-l(w)$, and $\mathscr{F}:=H_{q^{-1} Y(w)}^{m}\left(\mathcal{O}_{X}\right)=R \Gamma_{q^{-1 Y(w)}}\left(\mathcal{O}_{X}\right)[m]$. (Recall that $w \equiv\left(W / W_{I}\right)_{l}$.) Then $\mathscr{I} \cong \mathcal{C}$. Note that, for $i \geqq 0$,

$$
R \Gamma_{Z_{i}^{\prime} / Z_{i+1}^{\prime}} R \Gamma_{q^{-1 Y(w)}}=R \Gamma_{\left(Z_{i}^{\prime}-Z_{i+1}^{\prime}\right) \cap q^{-1 Y(w)}}=\underset{w \in W_{I}^{(i)}}{\bigoplus_{X(w . x)}}
$$

in $C$, and $\operatorname{codim}_{X} X(w x)=m+l(x)$ for $x \leqq W_{I}$. Hence, for $i \geqq 0$,

$$
H_{Z_{i}^{\prime} / Z_{i+1}^{\prime}}^{i}(\mathscr{I})=H_{Z_{i}^{\prime} / Z_{i+1}^{\prime}}^{i}\left(H_{q^{-1} Y(w)}^{m}\left(\mathcal{O}_{X}\right)\right)=\bigoplus_{x \in W_{I}^{(i)}} H_{X(w x)}^{m+\imath}\left(\mathcal{O}_{X}\right)
$$

and

$$
H_{Z_{i}^{\prime} / Z_{i+1}^{\prime}}^{j}(\mathscr{I})=0, \quad \text { if } j \neq i .
$$

By $[22,10.5], \Phi$ is locally Cohen-Macaulay with respect to $\left\{Z_{i}^{\prime}\right\}$. In other words, the complex (7.1.2) is exact for $\mathscr{F}^{\prime}=\mathscr{F}$. By $[22,9.5 .(\mathrm{e})], H_{Z_{i}^{\prime} / Z_{i+1}^{\prime}}^{\prime}(\mathscr{F})$ are $\Gamma(X,-)$-acyclic. Hence $H^{i}(X, \mathscr{I})$ are the cohomologies of the complex

$$
\Gamma\left(X, H_{Z_{0}^{\prime} / Z_{1}^{\prime}}^{0}(\mathscr{I})\right) \longrightarrow \Gamma\left(X, H_{Z_{1}^{\prime} / Z_{2}^{\prime}}^{1}(\mathscr{I})\right) \longrightarrow \cdots .
$$

Again by $[22,9.5 .(\mathrm{e})]$, the complex $(7.3 .3)$ can be naturally identified with the complex

$$
H_{Z_{0}^{\prime} / Z_{1}^{\prime}}^{0}(X, \mathscr{I}) \longrightarrow H_{Z_{1}^{\prime} / Z_{2}^{\prime}}^{1}(X, \mathscr{F}) \longrightarrow \cdots .
$$

By the same calculation as (7.3.1), we get

$$
H_{Z_{i}^{\prime} / Z_{i+1}^{\prime}}^{i}(X, \mathscr{I})=\bigoplus_{x \in W_{I}^{(i)}} H_{X(w x)}^{m+i}\left(X, O_{X}\right) .
$$

Since

$$
\begin{aligned}
& R \Gamma(X, \mathscr{F})=R \Gamma\left(X, R \Gamma_{q-1 Y(w)}\left(\mathcal{O}_{X}\right)\right)[m]=R \Gamma\left(Y, R q_{*} R \Gamma_{q-1 Y(w)}\left(\mathcal{O}_{X}\right)\right)[m] \\
= & R \Gamma\left(Y, R \Gamma_{Y(w)} R q_{*}\left(\mathcal{O}_{X}\right)\right)[m]=R \Gamma\left(Y, R \Gamma_{Y(w)}\left(\mathcal{O}_{Y}\right)\right)[m]=R \Gamma_{Y(w)}\left(Y, \mathcal{O}_{Y}\right)[m],
\end{aligned}
$$

we have

$$
H^{i}(X, \mathscr{I})= \begin{cases}H_{Y(w)}^{m}(Y, \mathcal{O}), & \text { if } i=0 \\ 0, & \text { if } i \neq 0 .\end{cases}
$$

Since the cohomologies of the complex (7.3.4) can be identified with (7.3.6), and each term of this complex are given by (7.3.5), we get the exact sequence

$$
0 \longrightarrow H_{Y(w)}^{m}\left(Y, \mathcal{O}_{Y}\right) \longrightarrow A^{0} \longrightarrow A^{1} \longrightarrow \cdots \longrightarrow A^{l^{\prime}} \longrightarrow 0,
$$

where

$$
A^{i}=\bigoplus_{x \in W_{I}^{(i)}} H_{X(w . x)}^{m+i}\left(X, \mathcal{O}_{X}\right) .
$$


Lemma 7.4. For $\lambda \in \mathrm{t}_{I, a d}^{2}$, we have an exact sequence

$$
0 \longleftarrow H_{Y(w)}^{m}\left(Y, \mathcal{O}_{Y}(\lambda)\right)^{*} \longleftarrow B_{0} \longleftarrow B_{1} \longleftarrow \cdots \longleftarrow B_{l^{\prime}} \longleftarrow 0,
$$

where $l^{\prime}=l\left(w_{I}\right)$ and

$$
B_{\imath}=\underset{x \in W_{I}^{(i)}}{\bigoplus_{i}} M(w x(\lambda-\rho)-\rho) .
$$

Proof. As a dual of (7.3.7), we get the exact sequence

$$
0 \longleftarrow H_{Y(w)}^{m}\left(Y, O_{Y}(\lambda)\right)^{*} \longleftarrow\left(A^{0}\right)^{*} \longleftarrow \cdots \longleftarrow\left(A^{l^{\prime}}\right)^{*} \longleftarrow 0 .
$$

Thus the assertion follows from (3.8).

Using (6.6), the above assertion can be also stated as follows.

Lemma 7.5. If $\lambda \subseteq \mathrm{t}_{\text {I, add }}$, we have an exact sequence of $U(\mathrm{~g})$-modules

$$
0 \longleftarrow V(w, \lambda, \mathfrak{p}) \longleftarrow B_{0} \longleftarrow B_{1} \longleftarrow \cdots \longleftarrow B_{l} \longleftarrow 0
$$

where $l^{\prime}$ and $B_{i}$ are as in (7.4).

Lemma 7.6. For $\lambda \equiv \mathrm{f}_{I, a d}^{2}, \operatorname{dim} \operatorname{Tor}_{i}^{1-}-(\boldsymbol{C}, V(w, \lambda, \mathfrak{p}))=\operatorname{card} W_{I}^{(2)}$, where $\boldsymbol{C}$ is considered as a trivial right $\mathfrak{n}_{-}-$module.

Proof. Since (7.5.1) gives a free $U\left(\mathfrak{n}_{-}\right)$-resolution of $V(w, \lambda, \mathfrak{p})$, the torsion groups are the homology groups of the complex

$$
0 \longleftarrow B_{0} / \mathfrak{n}_{-} B_{0} \stackrel{\overline{d_{1}}}{\longleftarrow} B_{1} / \mathfrak{n}_{-} B_{1} \stackrel{\overline{d_{2}}}{\longleftarrow} \cdots \stackrel{\overline{d_{l^{\prime}}}}{\longleftarrow} B_{l^{\prime}} / \mathfrak{n}_{-} B_{l^{\prime}} \longleftarrow 0 .
$$

For $x \in W_{I}^{(i)}$ and $y \in W_{I}^{(i-1)}$, we have $(w y(\lambda-\rho)-\rho)-(w x(\lambda-\rho)-\rho)=w((\rho-y \rho)$ $-(\rho-x \rho))$, which can not be equal to zero by $[2,9.8]$. Hence $\bar{d}_{2}=0$. (Note that $B_{i} / \mathfrak{n}_{-} B_{i} \cong \bigoplus_{x \in W_{I}^{(i)}} \boldsymbol{C} u(w x(\lambda-\rho)-\rho)$, where $u(-)$ is the highest weight vector of the Verma module $M(-)$.) Since $\operatorname{dim} B_{\imath} / \mathfrak{n}_{-} B_{\imath}=$ card $W_{I}^{(i)}$, we get the assertion.

Lemma 7.7. ([3]. Cf. [9].) For any field $k$ of characteristic zero, and $\lambda, \mu \in \mathrm{t}_{k}$, the following conditions are equivalent: (1) $M_{k}\left(\lambda-\rho, \mathfrak{b}_{k}\right) \supset M_{k}\left(\mu-\rho, \mathfrak{b}_{k}\right)$. (2) There is a sequence $\gamma_{1}, \cdots, \gamma_{n}$ of roots such that $\lambda \geqq r_{\gamma_{1}}(\lambda) \geqq r_{r_{2}} r_{\gamma_{1}}(\lambda) \geqq \cdots \geqq r_{r_{n}} \cdots$ $r_{\gamma_{2}} r_{\gamma_{1}}(\lambda)=\mu$.

7.8. For $x, y \in W$ and $\gamma \subseteq R$, we write $\underset{r}{\rightarrow} y$ if $x r_{\gamma}=y$ and $l(x)+1=l(y)$. Sometimes we shall omit the symbol $\gamma$ on the arrow. For $x, y \in W$, the following conditions are known to be equivalent (cf. [4]): (1) There exists a sequence $\gamma_{1}, \cdots, \gamma_{n}$ in $R$ such that $x=x_{0} \stackrel{r_{1}}{\rightarrow} x_{1} \stackrel{\gamma_{2}}{\rightarrow} \cdots \stackrel{\gamma_{n}}{\rightarrow} x_{n}=y$. (2) $x \leqq y . \quad$ (3) $\overline{B x B} \subset \overline{B y B}$.

Lemma 7.9. Let $\gamma \in R, w \in W$ and $w^{\prime}=w r_{r}$. Then $w \stackrel{r}{\rightarrow} w^{\prime}$ if and only if 
$w(-\rho) \leqq w^{\prime}(-\rho)$

Proof. Let $n=\left\langle\gamma^{2}, \boldsymbol{\rho}\right\rangle$. Then $0 \neq n \subseteq \boldsymbol{Z}$ and $w \rho-w^{\prime} \rho=n w(\gamma)$. Hence $\left.w \boldsymbol{\rho}\right\rangle$ $w^{\prime} \rho$ if and only if $n w(\gamma)>0$. Let $\gamma^{\prime}=w(\gamma)$. We may assume that $\gamma^{\prime}>0$ by replacing $\gamma$ with $-\gamma$ if necessary. Since $w^{\prime}=r_{\gamma^{\prime}} w$ and $w \rho-r_{\gamma} w \rho=n \gamma^{\prime}$, the condition $n \gamma^{\prime}>0$, i. e., $n>0$ is equivalent to $r_{\gamma^{\prime}} w>w$, i. e., $\underset{r}{\rightarrow} w^{\prime}$ by $[2,8.10]$.

Lemma 7.10. For $w, w^{\prime} \equiv W$, the following conditions are equivalent: (1) $M_{K}(w(\eta-\rho)-\rho, \mathfrak{b}) \supset M_{K}\left(w^{\prime}(\eta-\rho)-\rho, \mathfrak{b}\right)$. (Recall that $\eta=c \otimes_{A} K$.) (2) $M(-w \rho-$ $\rho, \mathfrak{b}) \supset M\left(-w^{\prime} \rho-\rho, \mathfrak{b}\right)$ and $w W_{I}=w^{\prime} W_{I}$. (3) $w \geqq w^{\prime}$ and $w W_{I}=w^{\prime} W_{I}$. (4) There is a sequence $\gamma_{1}, \cdots, \gamma_{n}$ in $R_{I}$ such that $w=w_{0} \stackrel{\gamma_{1}}{\longleftarrow} w_{1} \stackrel{\gamma_{2}}{\leftarrow} \stackrel{\gamma_{n}}{\leftarrow} w_{n}=w^{\prime}$.

Proof. Assume that $M_{K}(w(\eta-\rho)-\rho, \mathfrak{b}) \supset M_{K}\left(w^{\prime}(\eta-\rho)-\rho, \mathfrak{b}\right)$. By (7.7), there exists a sequence $\gamma_{1}^{\prime}, \cdots, \gamma_{n}^{\prime}$ in $R$ such that $w(\eta-\rho) \geqq r_{\gamma_{1}^{\prime}} w(\eta-\rho) \geqq \cdots \geqq$ $r_{\gamma_{n}^{\prime}} \cdots \gamma_{\gamma_{2}^{\prime}} r_{\gamma_{1}^{\prime}} w(\eta-\rho)=w^{\prime}(\eta-\rho)$. For any $x, y \cong W, x(\eta-\rho) \geqq y(\eta-\rho)$ implies that $x W_{I}=y W_{I}$ and $x(-\rho) \geqq y(-\rho)$. Hence $w W_{I}=w^{\prime} W_{I}$ and

$$
-w \rho \geqq-r_{\gamma_{1}^{\prime}} w \rho \geqq \cdots \geqq-r_{\gamma_{n}^{\prime}} \cdots r_{\gamma_{2}^{\prime}} r_{\gamma_{1}^{\prime}} w \rho=-w^{\prime} \rho .
$$

Let $w^{-1}\left(\gamma_{i}^{\prime}\right)=\gamma_{i}$. Since $w W_{I}=r_{\gamma_{i}^{\prime}} \cdots r_{\gamma_{1}^{\prime}} w W_{I}=w r_{\gamma_{i}} \cdots r_{\gamma_{1}} W_{I}$, the reflections $r_{\gamma_{i}}$ belong to $W_{I}$. Hence $\gamma_{i} \in R_{I}$ and $-w \rho \geqq-w r_{\gamma_{1}} \rho \geqq \cdots \geqq-w r_{\gamma_{n}} \cdots r_{\gamma_{2}} r_{\gamma_{1}} \rho=-w^{\prime} \rho$. By (7.9), this relation is equivalent to the assertion (4). Thus we have proved the implication $(1) \Rightarrow(4)$. The implications $(4) \Rightarrow(1)$ and $(2) \Leftrightarrow(4)$ can be proved in a similar way. Let us prove $(3) \Rightarrow(1)$. Take $x \in\left(W / W_{I}\right)_{s}$ so that $x W_{I}=$ $w W_{I}=w^{\prime} W_{I}$. If $w \geqq w^{\prime}$, then $x, w$ and $w^{\prime}$ can be expressed as $x=t_{1} \cdots t_{a}$, where $a=l(x)$ and $t_{i}: \Xi S, w=t_{1} \cdots t_{a} r_{1} \cdots r_{b}$, where $a+b=l(w)$ and $r_{j} \boxminus I$, and $w^{\prime}=t_{i_{1}} \cdots t_{i_{a}}, r_{j_{1}} \cdots r_{j_{b^{\prime}}}$, where $1 \leqq i_{1}<\cdots<i_{a^{\prime}} \leqq a, 1 \leqq j_{1}<\cdots<j_{b^{\prime}} \leqq b$ and $a^{\prime}+b^{\prime}=l\left(w^{\prime}\right)$. Since $x \equiv\left(W / W_{I}\right)_{s}$ and $t_{i_{1}} \cdots t_{i_{a}}, W_{I}=w^{\prime} W_{I}=x W_{I}=t_{1} \cdots t_{a} W_{I}$, the expression of $w^{\prime}$ should be $w^{\prime}=t_{1} \cdots t_{a} r_{j_{1}} \cdots r_{j_{b}}$. Let $y_{0}=r_{1} \cdots r_{b}$. Since $r_{1} \cdots r_{b} \geqq r_{j_{1}} \cdots r_{j_{b}}$, we can find a sequence $\gamma_{1}, \cdots, \gamma_{n} \cong R_{I}$ such that

$$
r_{1} \cdots r_{b}=y_{0} \longleftarrow \gamma_{1} \stackrel{r_{2}}{\longleftarrow} \cdots \stackrel{r_{n}}{\longleftarrow} y_{n}=r_{j_{1}} \cdots r_{j_{b}} .
$$

(Apply (7.8) to the Weyl group $W_{I}$ with the set of simple reflections $I$.) Then

$$
w=x y_{0} \stackrel{\gamma_{1}}{\longleftarrow} x y_{1} \stackrel{\gamma_{2}}{\longleftarrow} \cdots \stackrel{r_{n}}{\longleftarrow} x y_{n}=w^{\prime},
$$

and $y_{i} \Leftarrow W_{I}$. Since $\eta$ is $W_{I}$-invariant,

$$
x y_{i-1}(\eta-\rho)-x y_{i}(\eta-\rho)=x y_{i-1}(-\rho)-x y_{2}(-\rho) \text {. }
$$

By (7.9) and (7.10.2), $x y_{i-1}(-\rho) \geqq x y_{i}(-\rho)$. By $(7.10 .3), w(\eta-\rho)=x y_{0}(\eta-\rho) \geqq$ $\cdots \geqq x y_{n}(\eta-\rho)=w^{\prime}(\eta-\rho)$. Thus, by $(7.7)$, we get the implication $(3) \Rightarrow(1)$. The implication (4) $\Rightarrow$ (3) follows from (7.8). 
Lemma 7.11. Let $R=R(x)$ be an $n \times$ m-matrix with components in $\boldsymbol{C}[x]=$ $C\left[x_{1}, \cdots, x_{l}\right]$, where $x_{1}, \cdots, x_{l}$ are indeterminates. Assume that the rank of $R(\lambda)$ is $m-1$ for any $\lambda \in C^{l}$. Then there exists a unique vector $u=u(x) \equiv C[x]^{m}$ up to a non-zero constant multiple such that $R(x) u(x)=0$ and $u(x)$ is not divisible by any element of $\boldsymbol{C}[x] \backslash \boldsymbol{C}$. Moreover, $u(\lambda) \neq 0$ for any $\lambda \in \boldsymbol{C}^{l}$.

Proof. The assertion concerning the existence and the uniqueness is obvious. Let $R_{j}(x)(1 \leqq j \leqq m)$ be the column vectors of $R(x)$. For any $\lambda \in C^{l}$, there exists a unique nontrivial linear relation $\sum_{j=1}^{m} c_{j} R_{j}(\lambda)=0$ up to a constant multiple. Let $U_{k}=\left\{\lambda \equiv \boldsymbol{C}^{l}{ }_{\mid} c_{k} \neq 0\right\}$. For any $\lambda \subseteq U_{k}$, there exist unique $u_{j}^{\prime}(\lambda) \equiv$ $C(j \neq k)$ such that $R_{k}(\lambda)=\sum_{j \neq k} u_{j}^{\prime}(\lambda) R_{j}(\lambda)$. Using the formula of Cramer for a system of linear equations, we can express $u_{j}^{\prime}(x)$ 's as regular functions in $x \equiv$ $U_{k}$. By multiplying the denominators of $u_{j}^{\prime}(x)$ 's, we get a relation of the form $\sum_{j=1}^{m} u_{j}^{\prime \prime}(x) R_{j}(x)=0$ with $u_{j}^{\prime \prime}(x) \fallingdotseq C[x]$ such that $u_{k}^{\prime \prime}(\lambda) \neq 0$ for any $\lambda \in U_{k}$. Dividing by the greatest common divisor, we may assume that $u_{j}^{\prime \prime}(x)$ 's are relatively prime. Since $u$ is a non-zero constant multiple of $u^{\prime \prime}, u(\lambda) \neq 0$ for any $\lambda \equiv C^{l}=$ $\bigcup_{k} U_{k}$.

Lemma 7.12. If the equivalent conditions of (7.10) are satisfied with $w$ and $w^{\prime}$, then there exists a $U_{A}(\mathfrak{g})$-homomorphism $\varphi: M_{A}\left(w^{\prime}(c-\rho)-\rho, \mathfrak{b}\right) \rightarrow M_{A}(w(c-\rho)$ $-\rho, \mathfrak{b})$ such that $\varphi \otimes_{A, \lambda} \boldsymbol{C} \neq 0$ for any $\lambda \in \mathrm{f}_{I}^{2}$. Such a homomorphism $\varphi$ is unique up to multiplication of a non-zero complex number.

Proof. It suffices to consider the case where $w=w^{\prime} r_{\alpha}$ with $\alpha \in R_{I} \cap R_{+}$ and $l(w)=l\left(w^{\prime}\right)+1$. Then $w^{\prime}(\alpha)=R_{+}$,

$$
w(c-\rho)-\rho=w^{\prime}(c-\rho)-\rho+n w^{\prime} \alpha \geqq w^{\prime}(c-\rho)-\rho
$$

with $n:=\left\langle\rho, \alpha^{2}\right\rangle$, and $M_{K}\left(w^{\prime}(\eta-\rho)-\rho, \mathfrak{b}\right) \subset M_{K}(w(\eta-\rho)-\rho, \mathfrak{b})$. Hence there is a non-zero element $u$ of $U_{K}\left(\mathfrak{n}_{-}\right)$such that

$$
\begin{aligned}
& {[H, u]=-\left\langle n w^{\prime} \alpha, H\right\rangle \quad \text { for } H \in \mathrm{t}, \text { and }} \\
& \mathfrak{n}_{+} u v=0
\end{aligned}
$$

where $v$ denotes the canonical generator of $M_{A}(w(c-\rho)-\rho, \mathfrak{b})$. Multiplying and/or dividing an element of $A$ if necessary, we may assume that $u \in U_{A}\left(\mathfrak{n}_{-}\right)$ and $a^{-1} u \notin U_{A}\left(\mathfrak{n}_{-}\right)$for any $a \in A \backslash C$. Identify $A$ with a polynomial ring $C\left[x_{1}, \cdots, x_{l}\right]$, let $U\left(\mathfrak{n}_{-}\right)\left(-n w^{\prime} \alpha\right)$ be the set of $u \equiv U\left(\mathfrak{n}_{-}\right)$satisfying (7.12.2), and fix a $\boldsymbol{C}$-basis of $U\left(\mathfrak{n}_{-}\right)\left(-n w^{\prime} \alpha\right)$. Then $U\left(\mathfrak{n}_{-}\right)\left(-n w^{\prime} \alpha\right) \otimes A$ can be identified with $A^{m}$, where $m=\operatorname{dim} U\left(\mathfrak{n}_{-}\right)\left(-n w^{\prime} \alpha\right)$. Let $u=u(x)={ }^{t}\left(u_{1}(x), \cdots, u_{m}(x)\right)$. As is seen from the proof of $[9,7.6 .12]$, the condition (7.12.3) on $u$ can be written as a system of linear equations in $u_{i}(x)(1 \leqq i \leqq m)$ with coefficients in $A=C\left[x_{1}, \cdots\right.$, $\left.x_{l}\right]$, say, 


$$
R(x) u(x)=0 .
$$

Since $w(\lambda-\rho)-\rho=w^{\prime}(\lambda-\rho)-\rho+n w^{\prime} \alpha \geqq w^{\prime}(\lambda-\rho)-\rho$ for any $\lambda \in \mathrm{f}_{I}^{2}$, dim Hom $\left(M\left(w^{\prime}(\lambda-\rho)-\rho, \mathfrak{b}\right), M(w(\lambda-\rho)-\rho, \mathfrak{b})\right)=1$ by $[9,7.6 .6$ and 7.6.23]. Hence the solution space of (7.12.4) is one-dimensional for any $\left(\lambda_{1}, \cdots, \lambda_{l}\right) \in \mathbb{C}^{l}$, i. e., the rank of $R(\lambda)$ is equal to $m-1$. Thus applying (7.11) to our situation, we get an element $v^{\prime}=u v$ of $M_{A}(w(c-\rho)-\rho, \mathfrak{b})$ such that $v^{\prime} \otimes_{A, \lambda} 1 \neq 0$ for any $\lambda \subseteq \mathfrak{f}_{I}^{2}$. Then the $U_{A}(\mathrm{~g})$-homomorphism which sends the canonical generator of $M_{A}\left(w^{\prime}(c-\rho)\right.$ $-\rho, \mathfrak{b})$ to $v^{\prime}$ satisfies the condition.

7.13. Fix a reduced expression of each $w \Subset W_{I} \backslash\{e\}$ and let $\sigma(w) \in I$ be the last factor of the fixed expression. For any arrow $w \rightarrow w^{\prime}$, define the function $s\left(w, w^{\prime}\right)$ by the induction on $l(w)$ as follows. If $w \sigma\left(w^{\prime}\right)>w$, let $s\left(w, w^{\prime}\right)=1$. (Especially $s\left(e, w^{\prime}\right)=1$.) If $w \sigma\left(w^{\prime}\right)<w$, let $s\left(w, w^{\prime}\right)=-s\left(w \sigma\left(w^{\prime}\right), w^{\prime} \sigma\left(w^{\prime}\right)\right)$.

Lemma 7.14. ([2,10.3 and 10.4]) (1) If $w_{1} \rightarrow w_{3} \rightarrow w_{2}$ with $w_{i} \in W_{I}$, there exists exactly one $w_{4} \in W_{I}-\left\{w_{3}\right\}$ such that $w_{1} \rightarrow w_{4} \rightarrow w_{2}$. (2) For any quadruple $\left(w_{1}, w_{2}, w_{3}, w_{4}\right)$ as in (1), $s\left(w_{1}, w_{3}\right) s\left(w_{3}, w_{2}\right)+s\left(w_{1}, w_{4}\right) s\left(w_{4}, w_{2}\right)=0$.

7.15. Let $w \in\left(W / W_{I}\right)_{l}$. For any $x \in W_{I}, M_{K}(w x(\eta-\rho)-\rho, \mathfrak{b})$ can be embedded in $M_{K}(w(\eta-\rho)-\rho, \mathfrak{b})$ by $(7.10)$. Let $c^{\prime}(e, x)\left(x \subseteq W_{I}\right)$ be such embeddings. By (7.12), we may assume that $c^{\prime}(e, x)$ induces an embedding $c_{A}^{\prime}(e, x)$ of $M_{A}(w x(c-\rho)-\rho, \mathfrak{b})$ into $M_{A}(w(c-p)-\rho, \mathfrak{b})$, and that $c_{A}^{\prime}(e, x) \otimes_{A, \lambda} \boldsymbol{C} \neq 0$ for any $\lambda \in \mathrm{t}_{I}^{2}$. Fix such an embedding for each $x:=W_{I}$. Then, if $x_{1}, x_{2} \in W_{I}$ and $x_{1} \rightarrow x_{2}$, there is a unique embedding $c^{\prime}\left(x_{1}, x_{2}\right): M_{K}\left(w x_{2}(\eta-\rho)-\rho, \mathfrak{b}\right) \rightarrow M_{K}\left(w x_{1}(\eta\right.$ $-\rho)-\rho, \mathfrak{b})$, which is compatible with the fixed embeddings $c^{\prime}(e, x)$. Put $x_{0}=$ $e, M_{i}=M_{A}\left(w x_{i}(c-\rho)-\rho, \mathfrak{b}\right)(i=0,1,2)$, and let $u_{i}$ be the canonical generator of $M_{i}$. By (7.12), we can take $t \in K^{\times}$so that $u_{2}^{\prime}:=t c^{\prime}\left(x_{1}, x_{2}\right)\left(u_{2}\right) \leqq M_{1}$ and $u_{2}^{\prime} \otimes_{A, \lambda} 1 \neq 0\left(\in M_{1} \otimes_{A, \lambda} C\right)$ for any $\lambda \in \mathrm{I}_{I}^{\prime}$. Then $t c^{\prime}\left(e, x_{2}\right)$ sends $u_{2}$ to $c_{A}^{\prime}\left(e, x_{1}\right)\left(u_{2}^{\prime}\right)$ $\in M_{0}$. Hence $t c^{\prime}\left(e, x_{2}\right)$ sends $M_{2}$ into $M_{0}$, and for any $\lambda \in \mathrm{f}_{I}^{r},\left(t c^{\prime}\left(e, x_{2}\right) \otimes_{A, \lambda} C\right)$ $\left(u_{2} \otimes 1\right)=\left(c_{A}^{\prime}\left(e, x_{1}\right) \otimes_{1, \lambda} C\right)\left(u_{2}^{\prime} \otimes 1\right) \neq 0$, since $c_{A}^{\prime}\left(e, x_{1}\right) \otimes C$ is an embedding of a Verma module. Then by the uniqueness part of (7.12), $t \in C^{\times}$. Hence $c^{\prime}\left(x_{1}, x_{2}\right)$ sends $M_{A}\left(w x_{2}(c-\rho)-\boldsymbol{\rho}, \mathfrak{b}\right)$ into $M_{A}\left(w x_{1}(c-\boldsymbol{\rho})-\boldsymbol{\rho}, \mathfrak{b}\right)$ and $c^{\prime}\left(x_{1}, x_{2}\right) \otimes_{A, \lambda} C \neq 0$ for any $\lambda \in \mathrm{t}_{I}^{2}$. Let

$$
c\left(x_{1}, x_{2}\right)= \begin{cases}s\left(x_{1}, x_{2}\right) c^{\prime}\left(x_{1}, x_{2}\right), & \text { if } x_{1} \rightarrow x_{2}, \\ 0, & \text { otherwise }\end{cases}
$$

Let $W_{I}^{(i)}=\left\{x \in W_{I} \mid l(x)=i\right\}, l^{\prime}=l\left(x_{I}\right)$ as before, and

$$
C_{A, 2}=\bigoplus_{x \in W_{I}^{(i)}} M_{A}(w x(c-\rho)-\rho, \mathfrak{b}) .
$$

Then $d_{i}=(c(x, y))_{x \in W_{I}^{(2)}, y \in W_{I}^{(i+1)}}$ and the natural projection $\varepsilon: C_{A, 0}=M_{A}(w(c-\rho)$ $-\rho, \mathfrak{b}) \rightarrow V_{A}(w, c, \mathfrak{p})$ defines a sequence 


$$
0 \longleftarrow V_{A}(w, c, p) \longleftarrow C_{A, 0} \longleftarrow C_{A, 1} \longleftarrow \cdots \longleftarrow-C_{A, l^{\prime}} \longleftarrow 0
$$

which is a complex by (7.14). (Note that $C_{A, 1} \subset J_{A}(w(c-\rho)-\rho) \subset M_{A}(w(c-\rho)-$ $\rho, \mathfrak{b})=C_{A, 0}$.)

Theorem 7.16. For $w E\left(W / W_{I}\right)_{l}$, the complex

$$
0 \longleftarrow V_{K}(w, \eta, \mathfrak{p}) \longleftarrow C_{K, 0} \longleftarrow C_{K, 1} \longleftarrow \cdots \longleftarrow C_{K, l^{\prime}} \longleftarrow 0
$$

obtained as $(7.15 .1) \otimes_{A} K$ is exact.

Theorem 7.17. For $w \in\left(W / W_{I}\right)_{l}$ and $\lambda \in \mathrm{f}_{I}^{\check{L}}$, let

Then the complex

$$
C_{\imath}(\lambda)=\underset{x \in W_{I}^{(i)}}{\bigoplus^{(i)}} M(w x(\lambda-\rho)-\rho, \mathfrak{b})=C_{A, \imath} \otimes_{A, \lambda} C
$$

$$
0 \longleftarrow V(w, \lambda, \mathfrak{p}) \stackrel{\varepsilon}{\longleftarrow} C_{0}(\lambda) \stackrel{d_{1}}{\longleftarrow} C_{1}(\lambda) \longleftarrow \cdots \stackrel{u_{l^{\prime}}}{\longleftarrow} C_{l^{\prime}}(\lambda) \longleftarrow 0
$$

obtained as $(7.15 .1) \otimes_{A, \lambda} C$ is exact, if $\lambda \equiv \mathrm{t}_{I, a d}$.

Proof. Since (7.16) and (7.17) can be proved in the same way, we shall prove only (7.17). The surjectivity of $\varepsilon$ is obvious. Assume that we have already proved the exactness at $C_{0}(\lambda), \cdots, C_{2-1}(\lambda)$, and let us prove the exactness at $C_{\imath}(\lambda)$. (If $i=0$, we do nol assume anything.) Let $C_{j}=C_{j}(\lambda)$ and $K_{j}=\operatorname{ker} d_{j}$. The desired equality $d_{\imath+1}\left(C_{\imath+1}\right)=K_{i}$ is obtained by modifying the proof of $[2$, $\left.10.1^{\prime}\right]$. Here, we provisionally use notations close to those in [2]. Also in our case, it is enough to prove the same assertions as Lemmas 10.5, 10.6 and 10.7 of [2]. We do not need any modification concerning Lemma 10.5 of [2]. As in [2], the proof of "Lemma 10.6" is divided into following two steps.

Lemma a. $J H\left(K_{\imath}\right) \subset J H\left(C_{\imath+1}\right)$.

The proof is the same as in [2] except that we use the exact sequenece (7.5.1) instead of the one constructed in $[2,9.9]$.

Lemma b. Let $\lambda \in \mathrm{I}^{2}, M \in O$, and $L(\lambda-\rho)$ be the simple quotient of the Verma module $M(\lambda-\rho)=U(\mathrm{~g}) \otimes_{U(\mathrm{c})} C(\lambda-\rho)$. (See $[2, \S 8]$ for $\left.O\right)$. Assume that $\lambda$ is maximal in $\left\{\psi=\mathrm{t}^{2} \mid L(\psi-\rho)\right.$ occurs in $\left.J H(M)\right\}$. Let $\tau: M(\lambda-\rho) \rightarrow M$ be a homomorphism such that the image $\tau\left(f_{\lambda-\rho}\right)$ of the canonical generator $f_{\lambda-\rho}$ of $M\left(\lambda_{-} \rho\right)$ is not zero. Then the image of $\tau\left(f_{\lambda_{-}}\right)$in $M / \mathfrak{n}_{-} \cdot M$ is also not zero.

Proof. We shall prove the assertion by the induction on the length of $M$. Let $f_{\dot{\varphi}-\rho} \in M$ be a weight vector whose weight $\phi-\rho$ is maximal among the weights of $M$ and $N \subset M$ the submodule generated by $f_{\psi-\rho}$. Concerning the case where $\tau\left(f_{\lambda-\rho}\right) \notin N$, we do not need any modification of the proof given in [2]. Assume that $\tau\left(f_{\lambda-\rho}\right) \subseteq N$. Then $L(\lambda-\rho) \in J H(N) \subset J H(M(\psi-\rho))$. Hence $\lambda \leqq \psi$. On the other hand, $L(\psi-\rho) \fallingdotseq J H(N) \subset J H(M)$. According to the condition of the lemma, we get $\lambda=\psi$. Since $\phi-\rho$ is a maximal weight of 
$M, \tau\left(f_{\lambda-\rho}\right) \notin \mathfrak{n}_{-} \cdot M$.

To complete the proof of "Lemma 10.6 ", i. e., the injectivity of the mapping $C_{\imath+1} / \mathfrak{n}_{-} \cdot C_{\imath+1} \rightarrow K_{\imath} / \mathfrak{n}_{-} \cdot K_{i}$, it suffices to apply Lemma b to the module $M=K_{\imath}$. Cf. the proof of Lemma 10.6 of [2].

Lastly, we modify the proof of Lemma 10.7 of [2], namely, we replace the proof of the identity $\operatorname{dim}_{C} C_{i+1} / \mathfrak{n}_{-} C_{\imath+1}=\operatorname{dim}_{C} K_{\imath} / \mathfrak{n}_{-} K_{i}<\infty$ with the following argument. Define the modules $C$ and $D$, and the morphisms $\eta, \vartheta, \bar{\eta}$ and $\bar{\vartheta}$ in the same way as in the proof of Lemma 10.7 of [2]. Then we get exact sequences $D \stackrel{\eta}{\rightarrow} C \stackrel{\tau}{\rightarrow} K_{\imath} \rightarrow 0$, and $C \stackrel{\vartheta}{\rightarrow} C_{i} \stackrel{d_{i}}{\rightarrow} K_{\imath-1} \rightarrow 0$. (The latter sequence should be replaced with $C \stackrel{\vartheta}{\rightarrow} C_{0} \stackrel{\varepsilon}{\rightarrow} V(w, \lambda, \mathfrak{p}) \rightarrow 0$, if $i=0$.) As in [2], using these sequences, we can show that $\operatorname{dim} K_{2} / \mathfrak{n}-K_{i}=\operatorname{dim} \operatorname{Tor}_{i+1}^{n-1}(C, V(w, \lambda, \mathfrak{p}))$. On the other hand, by (7.6), we get $\operatorname{dim} C_{\imath+1} / \mathfrak{n}_{-} C_{\imath+1}=\operatorname{card} W_{I}^{(i+1)}=\operatorname{dim} \operatorname{Tor}_{i+1}^{\prime \prime}(C, V(w, \lambda, \mathfrak{p}))$.

Remark 7.18. We assumed that $\lambda-\rho$ is anti-dominant in (7.17). The author does not know whether this condition is necessary or not. A deeply related result is obtained by $\mathrm{O}$. Gabber and A. Joseph [10].

\section{§ 8. D-modules Associated to Complex Powers of Functions}

8.0. The purpose of this section is to prove (8.4), which will be used in the next section.

8.1. Let $X$ be a connected smooth variety over $C, \mathcal{O}=\mathcal{O}_{X}$ the sheaf of regular functions, $\mathscr{D}_{X}$ the sheaf of algebraic differential operators, $f_{1} \cdots, f_{l} \in$ $\Gamma\left(X, \mathcal{O}_{X}\right)$ which are not identically zero, $I=\{1,2, \cdots, l\}, e_{k}=(0, \cdots, 0,1,0, \cdots$, $0) \subseteq \boldsymbol{C}^{l}$, where 1 appears as the $k$-th component, $\underline{s}=\left(s_{1}, \cdots, s_{l}\right)$ the linear coordinate functions of $C^{l}, g=\prod_{i=1}^{l} f_{\imath}, B$ an open ball contained in $\Omega=X \backslash g^{-1}(0)$, and $\underline{f}^{s}=f_{1}^{s_{1} \ldots f_{l} s_{l}}$ a single valued branch of $B \times C^{l} \ni(x, \underline{s}) \rightarrow f_{1}(x)^{s_{1}} \ldots f_{l}(x)^{s_{i}}$. Let

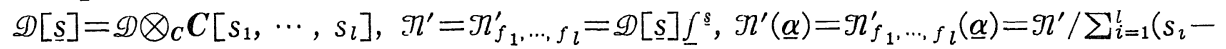
$\left.\alpha_{i}\right) \Re^{\prime}, \Re(\underline{\alpha})=\Re^{\prime}(\underline{\alpha})\left[f_{1}^{-1}, \cdots, f_{l}^{-1}\right]$, for $\underline{\alpha}=\left(\alpha_{1}, \cdots, \alpha_{l}\right) \equiv C^{l}$ and $u(\underline{\alpha})$ (resp. $u^{\prime}(\underline{\alpha})$ ) the section of the $\mathscr{D}$-module $\Re(\underline{\alpha})\left(\right.$ resp. $\left.\Re^{\prime}(\underline{\alpha})\right)$ corresponding to $\underline{f}^{s}$.

Lemma 8.2. For any $k \in I$, there exist $P_{k}=P_{k}(\underline{s}) \in \mathscr{D}[\underline{s}]$ and $b_{k}(\underline{s}) \subseteq C[\underline{s}]$ such that $P_{k} \underline{f}^{\underline{s}+e_{k}}=b_{k}(\underline{s}) \underline{f}^{\underline{s}}$ and $b_{k}$ is a product of polynomials of degree at most 1 .

Proof. This lemma is essentially due to Sabbah [28]. Since Sabbah works in the analytic category, we need to deduce from it the corresponding assertion in the algebraic category. The necessary argument is the same as the last part of the proof of $[12,2.5 .4]$.

Lemma 8.3. Let $g=\Pi f_{i}$ as above,

$$
W^{\prime}=\left\{(x, s \operatorname{grad} \log g(x)) \in T^{*} \Omega \mid s \in C^{\times}\right\},
$$




$$
\begin{aligned}
& \boldsymbol{W}=\text { the Zariski closure of } \boldsymbol{W}^{\prime} \text { in } T^{*} X, \text { and } \\
& \boldsymbol{W}_{0}=\left\{(x, y) \in \boldsymbol{W} \mid g(x) y_{1}=\cdots=g(x) y_{n}=0\right\} .
\end{aligned}
$$

For any $\underline{\alpha} \equiv C^{l}$, the characteristic variety of $\Re(\underline{\alpha})$ is $\boldsymbol{W}_{0}$. Especially $\Re(\alpha)$ is holonomic. (Here $T^{*}, X$ denotes the cotangent bundle of $X$.)

Proof. If $g^{-1}(0)$ is normal crossing, the assertion can be easily verified. Moreover, we can show that the characteristic cycle of $\mathscr{n}(\alpha)$ does not depend on $\alpha$ in this case. In general, by the Hironaka's desingularization theorem [15], there exist a smooth algebraic variety $\tilde{X}$ and a projective morphism $p$ : $\tilde{X} \rightarrow X$ such that $(g \circ p)^{-1}(0)$ is normal crossing and $p$ induces an isomorphism $p: \tilde{\Omega}:=\tilde{X} \backslash(g \circ p)^{-1}(0) \rightarrow X \backslash g^{-1}(0)=\Omega$. Let $\tilde{I}(\underline{\alpha})$ be the $\mathscr{D}_{\tilde{X}}$-module defined in the same way as $\Re(\alpha)$ using $f_{1} \circ p, \cdots, f_{l} \circ p$ instead of $f_{1}, \cdots, f_{l}$. Then $\Omega(\underline{\alpha})=$ $j_{*}(\Re(\underline{\alpha}) \mid \Omega)=j_{*}\left(\int_{p \mid \hat{\Omega}} \tilde{\Re}(\underline{\alpha}) \mid \tilde{\Omega}\right)=\int_{p} \tilde{j}_{*}(\tilde{\Pi}(\underline{\alpha}) \mid \tilde{\Omega})=\int_{p} \tilde{\Re}(\underline{\alpha})$, where $j: \Omega \rightarrow X$ and $\tilde{j}: \tilde{\Omega} \rightarrow$ $\tilde{X}$ are the inclusion mappings, and $\int_{p}$ is the integration along fibres. (See [17].) Since $\tilde{\Re}(\underline{\alpha})$ is holonomic, the characteristic cycle of $\Re(\underline{\alpha})=\int_{p} \tilde{N}(\underline{\alpha})$ depends only on the characteristic cycle of $\tilde{\eta}(\underline{\alpha})[23]$. Hence the characteristic cycle of $\Re(\underline{\alpha})=\eta_{f_{1}, \cdots, f_{l}}\left(\alpha_{1}, \cdots, \alpha_{l}\right)$ coincides with that of $\eta_{g}(0)$, whose support is known to be $W_{0}[29]$ (cf. $[12,2.4 .6,(2)]$ ).

The purpose of this section is to prove the following assertion.

Proposition 8.4. Let $p$ be a point of $W_{0}$, and assume that there exist invertible micro-differential operators $Q_{k}$ in a neighbourhood of $p$ such that

$$
Q_{k} \underline{\underline{s}}^{\underline{s}+e_{k}}=b_{k}(\underline{s}) \underline{f}^{\underline{s}},
$$

where $b_{k}$ 's are the polynomials appeared in (8.2). Then $\Re(\underline{\alpha})$ is a simple Dmodule (i.e., it does not have a non-trivial coherent $D$-submodule) if and only if $b_{k}(\underline{\alpha}-\underline{v}) \neq 0$ for any $k \in I$ and $\underline{v} \in \boldsymbol{Z}^{l}$.

8.5. Proof of the "if part". Let $\mathscr{M}$ be a coherent non-zero $\mathscr{D}$-submodule of $\mathscr{N}(\underline{\alpha})$. Since $\Re(\underline{\alpha})$ does not have a non-zero submodule supported by $g^{-1}(0)$ and since $\mathscr{N}(\underline{\alpha}) \mid\left(X \backslash g^{-1}(0)\right)$ is a simple $\mathscr{D}$-module, $\mathscr{M}=\mathscr{N}(\underline{\alpha})$ on $X \backslash g^{-1}(0)$. Let $u^{\prime \prime}(\underline{\alpha})$ be the element of $\mathscr{N}(\underline{\alpha}) / \mathscr{M}$ corresponding to $u(\underline{\alpha})$. Then $\mathscr{N}(\underline{\alpha}) / \mathscr{M}=\cup_{m \in Z}$ $\mathscr{D}\left(g^{m} u^{\prime \prime}(\underline{\alpha})\right)$. Fix an integer $m$ arbitrarily. Since the support of $\mathscr{N}(\underline{\alpha}) / \mathscr{M}$ is contained in $g^{-1}(0), g^{m+m^{\prime}} u^{\prime \prime}(\underline{\alpha})=0$ for a sufficiently large integer $m^{\prime}$. Applying $P_{k}$ 's several times to this relation, we get a relation of the form $\left(\Pi_{(k, v) \in S} b_{k}(\underline{\alpha}\right.$ $+\underline{v}) g^{m} u^{\prime \prime}(\underline{\alpha})=0$ with some finite subset $S$ of $I \times \boldsymbol{Z}^{l}$. It follows from our assumption that $g^{m} u^{\prime \prime}(\underline{\alpha})=0$ and $\mathscr{N}(\underline{\alpha})=\mathscr{M}$.

Remark 8.5.1. The assumptions on $Q_{k}$ 's are not used in the "if part". 
8.6. In order to prove the "only if part", we need some preliminaries.

8.6.1. Let $\mathscr{M}$ be a $\mathscr{D}_{X}$-module, $u$ a section of $\mathscr{M}$, and $f \equiv \mathcal{O}_{X}$. Consider the left ideal $\mathcal{I}$ of $\mathscr{D}[s]$ consisting of differential operators $P(s) \triangleq \mathscr{D}[s]$ such that $\left(f^{m-s} P(s) f^{s}\right) u=0$ holds in $C[s] \otimes_{c} \mathscr{M}$ for a sufficiently large integer $m$. (Note that $f^{m-s} P(s) f^{s} \in \mathscr{D}[s]$ if $m$ is sufficiently large.) Let $f^{s} u$ be the section of $\mathcal{L}:=\mathscr{D}[s] / \mathscr{I}$ corresponding to the identity element of $\mathscr{D}[s]$. Then $\mathcal{L}$ $=\mathscr{D}[s] / \mathscr{I}=\mathscr{D}[s]\left(f^{s} u\right)$. For a complex number $\alpha$, let $f^{c} u$ be the section of $\mathcal{L} /(s-\alpha) \mathcal{L}$ corresponding to $f^{s} u$. Then $\mathcal{L} /(s-\alpha) \mathcal{L}=\mathscr{D}\left(f^{\alpha} u\right)$.

8.6.2. Define an endomorphism $t$ of the $\mathscr{D}$-module $\mathcal{L}$ by $t: P(s)\left(f^{s} u\right) \rightarrow$ $P(s+1)\left(f \cdot f^{s} u\right)$. Then $t$ is well-defined and injective.

Proof. Let $m$ be a sufficiently large integer, and $f^{m-s} \cdot P(s) \cdot f^{s}=\sum_{\jmath \geqq 0} s^{j} P_{\jmath}$. The following conditions are equivalent: $P(s)\left(f^{s} u\right)=0 . \quad \sum_{J_{0} s^{j}} P_{j} u=0 . \quad \sum_{j \geqq 0}(s$ $+1)^{j} P_{j} u=0 . \quad P(s+1)\left(f \cdot f^{s} u\right)=0$. Thus we get the assertion.

8.6.3. If $\mathscr{D} u$ is holonomic, then $\mathcal{L}$ is a subholonomic $\mathscr{D}$-module, and $\mathcal{L} /(s$ $-\alpha) \mathcal{L}(\alpha \in C)$ and $\mathcal{L} / t \mathcal{L}$ are holonomic.

Proof. The first assertion is due to Kashiwara [18, Theorem 2.5]. The remaining assertions follow from it.

8.6.4. If $\mathscr{Q} u$ is holonomic, the composition factors of $\mathcal{L} /(s-\alpha) \mathcal{L}$ (including multiplicities) depend only on $(\alpha \bmod Z)$.

Proof. Let $C[s, t]$ be the $C$-algebra defined by the relation $t s=(s+1) t$, and $\mathscr{D}[s, t]=\mathscr{D} \otimes_{C} C[s, t]$. The multiplication by $s$ and the endomorphism $t$ defined in (8.6.2) give a $\mathscr{D}[s, t]$-module structure in $\mathcal{L}$. The assertion follows from (8.6.2), (8.6.3) and [12, 2.8.5].

8.6.5. Assume that there exist a differential operator $P(s)$ and a polynomial $c(s) \equiv \boldsymbol{C}[s]$ such that $P(s)\left(f \cdot f^{s} u\right)=c(s) f^{s} u$. If $c(\alpha-j) \neq 0$ for $j=1,2, \cdots$, then $(\mathcal{L} /(s-\alpha) \mathcal{L}=) \mathscr{D}\left(f^{\alpha} u\right)=\mathscr{D}\left(f^{\alpha} u\right)\left[f^{-1}\right]$ (cf. [12, 2.3.8]).

8.6.6. Let $p$ be a point of the conormal bundle $T^{*} X$, and assume that $p$ is contained in the characteristic variety of $\mathcal{L} /(s-\alpha) \mathcal{L}$ for any $\alpha \equiv C$. Let $\mathcal{E}_{p}$ be the ring of germs of micro-differential operators at $p$. If there exists a micro-differential operator $Q \boxminus \mathcal{E}_{p}$ which is invertible and satisfies $Q\left(f \cdot f^{s} u\right)=$ $c(s) f^{s} u$ with the same $c(s)$ as in (8.6.5), then $c(s)$ is a minimal polynomial of $s \in \operatorname{End}_{\mathscr{D}}(\mathcal{L} / t \mathcal{L})$.

Proof. Let $\mathcal{L}_{p}=\mathcal{E}_{p} \otimes_{\mathscr{D}} \mathcal{L}$. Since $p$ lies in the characteristic variety of $\mathcal{L} /(s-\alpha)_{\mathcal{L}} \mathcal{L}, 0 \neq \mathcal{E}_{p} \otimes_{\mathscr{D}} \mathcal{L} /(s-\alpha) \mathcal{L}=\mathcal{L}_{p} /(s-\alpha) \mathcal{L}_{p}$ for any $\alpha \in \mathbb{C}$. Hence $(s-\alpha) \mathcal{L}_{p}$ 
$\subsetneq \mathcal{L}_{p}$ for any $\alpha \sqsubseteq \boldsymbol{C}$. Since $a(s) \mathcal{L}_{p} \neq 0$ for any $a(s) \in C[s]-\{0\}$, the above relation implies the faithful flatness of $\mathcal{L}_{p}$ over $\boldsymbol{C}[s]$. Let $c_{1}(s)$ be a minimal polynomial of $s \leq E n d(\mathcal{L} / t \mathcal{L})$. Then $c(s)=c_{1}(s) d(s)$ with some $d(s) \lesssim C[s]$. If $d(s) \notin \boldsymbol{C}^{\prime}$, then $c(s) \boldsymbol{C}[s] \varsubsetneqq c_{1}(s) \boldsymbol{C}[s]$ and $c(s) \mathcal{L}_{p} \subsetneq c_{1}(s) \mathcal{L}_{p} \subset t \mathcal{L}_{p}=\mathcal{E}_{p}[s]\left(f^{s+1} u\right)$. On the other hand, since $Q$ is invertible in $\mathcal{E}_{p}, c(s) \mathcal{L}_{p}=c(s) \mathcal{E}_{p}[s]\left(f^{s} u\right)=\mathcal{E}_{p}[s]$ $Q\left(f^{s+1} u\right)=\mathcal{E}_{p}[s]\left(f^{s+1} u\right)$. Thus we get a contradiction. Hence $d(s) \cong \boldsymbol{C}^{\wedge}$, i. e., $c(s)$ is a minimal polynomial.

8.7. Proof of the "only if part". Assume that $b_{1}(\underline{\alpha}-\underline{v})=0$ for some $\underline{\underline{v}} \equiv \boldsymbol{Z}^{l}$. and let us prove that $\eta(\underline{\alpha})$ is not simple. Since $\eta(\underline{\alpha})=i_{*}(\eta(\underline{\alpha}) \mid \Omega)$ and $I_{l}(\underline{\alpha}) \mid \Omega$ depends only on $\left(\underline{\alpha} \bmod \boldsymbol{Z}^{l}\right)$, we may freely replace $\underline{\alpha}$ with other element in the same residue class modulo $\boldsymbol{Z}^{l}$. Especially, we may assume from the beginning that $b_{1}(\underline{\alpha})=0$. We have $\left(P_{1} P_{2} \cdots P_{l}\right) g^{s+1} u(\underline{\alpha})=c(s) g^{s} u(\underline{\alpha})$, where $c(s)=$ $\prod_{k=1}^{l} b_{k}\left(s+\alpha_{1}+1, \cdots, s+\alpha_{k-1}+1, s+\alpha_{k}, \cdots, s+\alpha_{l}\right)$. Put $\mathcal{L}=\mathscr{D}[s]\left(g^{s} u(\underline{\alpha})\right)$ and let us show that the conditions of (8.6.6) are satisfied. For any $\beta \in C$, there exists an integer $m$ such that

$$
\mathcal{L} /(s-\beta+m) \mathcal{L}=\mathscr{D}\left(g^{\beta-m} u(\underline{\alpha})\right)=\mathscr{D}\left(g^{\beta} u(\underline{\alpha})\right)\left[g^{-1}\right]=\mathfrak{l}(\underline{\alpha}+\beta \underline{\delta}),
$$

where $\underline{\delta}=(1, \cdots, 1)$. (Cf. (8.6.5).) By (8.6.4), the characteristic variety of $\mathcal{L} /(s$ $-\beta) \mathcal{L}$ coincides with that of $\mathcal{L} /(s-\beta+m) \mathcal{L}=\eta(\underline{\alpha}+\beta \underline{\delta})$, which is $W_{0}$ by (8.3). Moreover, $\left(Q_{1} Q_{2} \cdots Q_{l}\right) g^{s+1} u(\underline{s})=c(s) g^{s} u(\underline{s})$ and $Q_{1} Q_{2} \cdots Q_{l}$ is invertible at $p \subseteq \boldsymbol{W}_{0}$. Thus the conditions of (8.6.6) are satisfied. Hence $c(s)$ is a minimal polynomial of $s \in \operatorname{End}_{\mathscr{D}}\left(\mathcal{L} / t_{\alpha} \mathcal{L}\right)$. Since $b_{1}(\underline{\alpha})=0, c(0)=0$. Let $c(s)=c_{1}(s) s$. If $s$ is surjective, then $s$ is an automorphism of $\mathcal{L} / t \mathcal{L}$, for $\mathcal{L} / t \mathcal{L}$ is holonomic. But, then $c_{1}(s)$ $=0$ as an endomorphim of $\mathcal{L} / \mathcal{L} \mathcal{L}$, which contradicts the minimality of $c(s)$. Hence $s \subseteq \operatorname{End}(\mathcal{L} / t \mathcal{L})$ is not surjective, i. e., $s \mathcal{L}+t \mathcal{L} \subsetneq \mathcal{L}$. Then $\mathscr{I}:=(s \mathcal{L}+t \mathcal{L}) /$ $s \mathcal{L} \subsetneq \mathcal{L} / s \mathcal{L}$. Since $t \mathcal{L}=\mathcal{L}$ on $\Omega, \mathscr{I} \neq 0$. Thus we get a proper submodule of $\mathcal{L} / s \mathcal{L}$. Since $\mathcal{L} / s \mathcal{L}$ and $\mathcal{L} /(s+m) \mathcal{L}=\Re(\underline{\alpha})$ (cf. (8.7.1)) have the same composition factors, $\Re(\underline{\alpha})$ is not simple.

\section{$\S$ 9. Submodules of $V(w, \lambda, \downarrow)$ and $b$-functions}

9.0. The purpose of this section is to prove (9.4) and its corollary (9.13). In (9.4), we describe the submodule lattice of $V(w, \lambda, \mathfrak{p})$. In order to state (9.4), we need some definitions, which are given in (9.1)-(9.3). The proof of (9.4) is given in (9.5)-(9.11). In (9.13), we give a criterion for the simplicity of generalized Verma modules, by combining (8.4) and (9.4).

9.1. Let $\lambda$ be a $W_{I}$-invariant character. If we forget the $\mathscr{D}_{Y}(\lambda)$-module structure, then $\mathcal{O}\left(w U_{-}, \lambda\right)=\mathcal{O}_{Y} \mid w U_{-} \cdot y_{0}$. (See $(2.5)$ for $\mathcal{O}\left(w U_{-}, \lambda\right)$.) Hence we can regard $1 \equiv \Gamma\left(w U_{-} \cdot y_{0}, \mathcal{O}_{Y}\right)$ as a section of $\mathcal{O}\left(w U_{-}, \lambda\right)$, which we shall denote by $1_{w}^{\lambda}$. We write $1^{\lambda}$ for $1_{e}^{\lambda}$. Then $\mathscr{D}_{Y} \mid U_{-} \cdot y_{0}$ is isomorphic to $\mathscr{D}_{Y}(\lambda) \mid U_{-} \cdot y_{0}$ by 
$\mathscr{D}_{Y} \equiv P \rightarrow 1^{\lambda} \otimes P \otimes 1^{-\lambda}$. Thus from any $\left(\mathscr{D}_{Y}(\lambda) \mid U_{-} \cdot y_{0}\right)$-module $\mathscr{M}$, we get a $\left(\mathscr{D}_{Y} \mid U_{-} \cdot y_{0}\right)$-module $1^{-\lambda} \otimes \mathscr{M}$.

9.2. Semi-invariants. From now on, we assume that $G$ is semi-simple and simply connected. Note that these assumptions are not restrictive for the study of (generalized) flag manifolds, $U(\mathfrak{g})$-modules, etc. Then each $\widetilde{\sigma}=\sum_{i=1}^{l}$ $n_{\imath} \widetilde{\sigma}_{i}\left(n_{i} \in \boldsymbol{Z}\right)$ determines rational characters of $T, B$ and $B_{-}:=w_{S} B w_{S}$ (via the projection $B_{-} \rightarrow T$ ), which we shall denote by the same letter $\widetilde{\sigma}$. If every $n_{\imath}$ $(1 \leqq i \leqq l)$ is non-negative, then there exists a regular function $f^{\varpi}$ on $G$ such

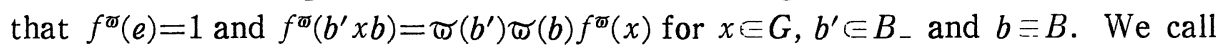
such a polynomial a semi-invariant. These functions $f^{\sigma}$ can be constructed as follows. Let $V_{\varpi}$ be a finite dimensional irreducible representation of $G$ with highest weight $\varpi, v_{\varpi}$ its highest weight vector, and $v_{-\varpi}$ the lowest weight vector of the contragradient representation $V_{\widetilde{\varpi}}^{\sim}$ of $V_{\varpi}$ such that $\left\langle v_{-\varpi}, v_{\varpi}\right\rangle=1$. Then the regular function $f^{\varpi}$ is given by

$$
f^{\varpi}(g)=\left\langle v_{-\varpi}, g v_{\varpi}\right\rangle .
$$

Let $f_{i}=f^{\varpi_{i}}$. Then $f^{\varpi}=\prod_{i=1}^{l} f_{i}^{n_{\imath}}$.

9.3. Assume that $\Pi_{I}=\left\{\alpha_{k+1}, \cdots, \alpha_{l}\right\}$. Then $t_{I}^{r}=\left\{\lambda=\sum_{i=1}^{k} \lambda_{2} \widetilde{\varpi}_{i} \mid \lambda_{2} \in C\right\}$. Let $s=\left(s_{i}\right)_{1 \leqq i \leqq k}$ be independent complex variables, $f^{s}(x)=\Pi_{i=1}^{k} f_{i}^{s_{i}}(x), \mathscr{\eta}^{\prime}=\mathscr{D}_{G}\left[s_{1}, \cdots\right.$, $\left.s_{k}\right] f^{s}, \Re^{\prime}(\lambda)=\eta^{\prime} / \sum_{i=1}^{k}\left(s_{i}-\lambda_{i}\right) \Re^{\prime}$, and $u(\lambda)$ the generator of $\eta^{\prime}(\lambda)$ corresponding to $f^{s}$. For any $\mathscr{D}_{G}$-module $\mathscr{M}$, let $g \mathscr{M}:=L_{g^{-1}}^{*} \mathscr{M}(g \equiv G)$, where $L_{g-1}$ is the left translation by $g^{-1}$.

Theorem 9.4. Assume that $\lambda=\lambda_{c}+\lambda_{d} \in \mathrm{f}_{r a d}^{2}$, where $\lambda_{c}$ is $W_{I}$-invariant and $\lambda_{d}$ is the lowest weight of a finite dimensional irreducible P-module. For $w=$ $\left(W / W_{I}\right)_{l}$, put

$$
\begin{aligned}
& L_{1}=\{U(\mathfrak{g}) \text {-submodules of } V(w, \lambda, \mathfrak{p})\}, \\
& L_{1}^{*}=\left\{U(\mathfrak{g}) \text {-submodules of } V(w, \lambda, \mathfrak{p})^{*}\right\}, \\
& L_{2}=\left\{\text { coherent } \mathscr{D}_{X}(\lambda) \text {-submodules of } H_{X(w)}^{c d(w)}\left(\mathcal{O}_{X}\left(\lambda_{c}\right)\right) \otimes \mathcal{O}_{X}\left(\lambda_{d}\right)\right\} \\
& L_{3}=\left\{\text { coherent } \mathscr{D}_{X}\left(\lambda_{c}\right) \text {-submodules of } H_{X(w)}^{c d(w)}\left(\mathcal{O}_{X}\left(\lambda_{c}\right)\right)\right\}, \\
& L_{4}=\left\{\text { coherent } \mathscr{D}_{Y}\left(\lambda_{c}\right) \text {-submodules of } H_{Y(w)}^{c d(w)}\left(\mathcal{O}_{Y}\left(\lambda_{c}\right)\right)\right\}, \\
& L_{5}=\left\{\text { coherent }\left(\mathscr{D}_{Y}\left(\lambda_{c}\right) \mid U_{-} \cdot y_{0}\right) \text {-submodules of } H_{Y(w)}^{c d(w)}\left(\mathcal{O}_{Y}\left(\lambda_{c}\right)\right) \mid U_{-} \cdot y_{0}\right\}, \\
& L_{6}=\left\{\text { coherent }\left(\mathscr{D}_{Y} \mid U_{-} \cdot y_{0}\right) \text {-submodules of } 1^{-\lambda_{c}} \otimes\left(H_{Y(w)}^{c d(w)}\left(\mathcal{O}_{Y}\left(\lambda_{c}\right)\right) \mid U_{-} \cdot y_{0}\right)\right\}, \text { and } \\
& L_{7}=\left\{\text { coherent } \mathscr{D}_{G^{-}} \text {-submodules of } H_{B w P}^{c d(w)}\left(w \eta^{\prime}\left(-\lambda_{c}\right)\right)\right\} .
\end{aligned}
$$

Then as lattice-ordered sets, 


$$
L_{1}^{o p p} \cong L_{1}^{*} \cong L_{2} \cong L_{3} \cong L_{4} \cong L_{5} \cong L_{6} \cong L_{7} \text {. }
$$

(See (1.1) for $\mathrm{t}_{r a d},\left(W / W_{I}\right)_{l}, U_{-}$, and *, (4.8) for $\mathcal{O}_{X}\left(\lambda_{d}\right)$, and (4.2) for $X, Y, X(w)$, $Y(w), c d(w)$ and $y_{0}$. We denote the dual of $L$ by $L^{o p p}$.)

Proof. Obviously, $L_{1}^{o p p} \cong L_{1}^{*}, L_{2} \cong L_{3}$ and $L_{5} \cong L_{6}$. By (3.4) and (6.6), $L_{1}^{*} \cong$ $L_{2}$. By $(5.4), L_{3} \cong L_{4}$. In order to prove $L_{4} \cong L_{5}$ and $L_{4} \cong L_{7}$, we need some preliminaries. Henceforth until the end of (9.11), we write $\lambda$ for $\lambda_{c}$, since we exclusively consider the $W_{I}$-invariant characters.

Lemma 9.5. For any $w \equiv W, Y(w) \vdash, U_{-} \cdot y_{0} \neq \phi$

Proof. Since $B w_{S} B$ is a Zariski open subset of $G, B w_{s} B g \cap B w_{S} B \neq \boldsymbol{\phi}$ for any $g \equiv G$. Hence $B g \cap w_{S} B w_{S} B \neq \dot{\phi}$. Especially, $Y(w) \cap_{1} U_{-} \cdot y_{0}=B w y_{0} \cap w_{S} B w_{S}$ $\cdot B y_{0} \neq \dot{\phi}$ for any $w \subseteq W$.

Lemma 9.6. Let $S$ be a smooth algebraic variety over $C, U$ a Zariski open subset of $S, A_{S}$ a twisted ring of algebraic differential operators on $S([19,2.3 .3])$, $A_{U}=A_{S} \mid U, M$ a coherent $A_{S}$-module, and $N^{\prime}$ a coherent $A_{U}$-submodule of $M \mid U$. Then there exists a coherent $A_{S}$-submodule $N$ of $M$ such that $N \mid U=N^{\prime}$.

Proof. Since $M$ and $N^{\prime}$ are quasi-coherent over $\Theta_{S}$ and $\mathcal{O}_{U}$, respectively, we can find a quasi-coherent $\mathcal{O}_{S}$-submodule $N_{1}$ of $M$ such that $N_{1} \mid U=N^{\prime}$, by $[11,(5.9 .2)]$. Let $N$ be the $A_{s}$-submodule of $M$ generated by $N_{1}$. Then $N$ is a coherent $A_{S}$-submodule of $M$ such that $N \mid U=N^{\prime}$.

9.7. Let us prove that $L_{4} \cong L_{5}$. Define a mapping $L_{4} \rightarrow L_{5}$ by the restriction to $U_{-} \cdot y_{0}$. By (9.6), this morphism is surjective. Assume that two modules $M$ and $\mathrm{V}$ in $L_{4}$ restricts to the same module in $L_{5}$. Then $I I /(M \cap N)$ is supported by the complement of $U_{-} \cdot y_{0}$. Hence every irreducible component of the characteristic variety of $M /(M \cap N)$ is the conormal bundle of some subvariety of $Y \backslash U_{-} \cdot y_{0}$. On the other hand, each irreducible component of the characteristic variety of $H_{Y(w)}^{c d(w)}\left(\mathcal{O}_{Y}(\lambda)\right)$ is the conormal bundle of $Y\left(w^{\prime}\right)$ with some $w^{\prime} \equiv W / W_{I}$, which is not a conormal bundle of a subvariety of $Y \backslash U_{-} \cdot y_{0}$ by (9.5). Hence $M /(M \cap N)=0$, i. e., $M=N$.

Thus it remains to prove that $L_{4} \cong L_{7}$.

Lemma 9.8. A defining equation of the hypersurface $\overline{B_{-} \cdot r_{\alpha_{i}} B}$ of $G$ is given by $f_{\imath}=0$.

Proof. Since $r_{\alpha_{j}}\left(\widetilde{\omega}_{\imath}\right)=\widetilde{\omega}_{\imath}-\delta_{\imath \jmath} \alpha_{\jmath}$, we can show that $f_{\imath}\left(r_{\alpha_{j}}\right) \neq 0$ (resp.=0) if $i \neq j$ (resp. $i=j$ ) by (9.2.1). Since $f_{\imath}^{-1}(0)$ is a union of cosets in $B_{-} \backslash G / B$, and is a hypersurface of $G$, we have $f_{\imath}^{-1}(0)=\overline{B_{-} \cdot r_{\alpha_{i}} B}$. For $A \in \mathfrak{g}\left(\alpha_{\imath}\right)$ and $A^{\prime} \in \mathfrak{g}\left(-\alpha_{\imath}\right)$ 
such that $\left[A, A^{\prime}\right]=\check{\alpha_{\imath}}$, we have

$$
\begin{aligned}
f_{\imath}\left(\exp (t A) r_{a_{i}}\right) & =\left\langle v_{-\varpi_{i}}, r_{\alpha_{i}} v_{\varpi_{i}}\right\rangle+t\left\langle v_{-\varpi_{i}}, A r_{\alpha_{i}} v_{\varpi_{i}}\right\rangle+O\left(t^{2}\right) \\
& =c t\left\langle v_{-\varpi_{i}}, A A^{\prime} v_{\widetilde{\varpi}_{i}}\right\rangle+O\left(t^{2}\right)
\end{aligned}
$$

with some $c \in C \backslash\{0\}$. (By the representation theory of $s l_{2}$, we can show that $A^{\prime} v_{\varpi_{i}} \neq 0$. Considering the weight of $r_{\alpha_{i}} v_{\varpi_{i}}$, we get $r_{\alpha_{i}} v_{\varpi_{i}}=c A^{\prime} v_{\varpi_{i}}(c \neq 0)$.) But $A A^{\prime} v_{\varpi_{i}}=\left[A, A^{\prime}\right] v_{\varpi_{i}}=\alpha_{\imath}^{2} v_{\varpi_{i}}=v_{\varpi_{i}}$. Hence $f_{i}\left(\exp (t A) r_{\alpha_{i}}\right)=c t+O\left(t^{2}\right)(c \neq 0)$. Thus $f_{\imath}=0$ is a defining equation of $\overline{B_{-} \cdot r_{\alpha_{i}} B}$.

Lemma 9.9. $G \backslash B_{-} \cdot P=\bigcup_{r \in S \backslash I} \overline{B_{-} \cdot r B}$.

Proof. The minimal elements of $W \backslash W_{I}$ are $S \backslash I$. Hence the maximal elements of $W \backslash w_{S} W_{I}$ are $\left\{w_{S} r \mid r \in S \backslash I\right\}$. By the equivalence $w \geqq w^{\prime} \Leftrightarrow \overline{B w B} \supset \overline{B w^{\prime} B}$, we ge $G \backslash B w_{S} P=\cup_{w \in W \backslash w_{S} W_{I}} B w B=\cup_{r \in S \backslash I} \overline{B w_{S} r B}$. Multiplying $w_{S}$ from the left, we get the assertion.

Lemma 9.10. The rational characters $\widetilde{\sigma}_{\imath}(1 \leqq i \leqq k)$ of $B$ can be extended to those of $P$ and $P_{\text {. }}$. Denoting them by the same letter $\widetilde{\sigma}_{\iota}$, we have $f_{i}\left(p^{\prime} g p\right)=$ $\widetilde{\varpi}_{\imath}\left(p^{\prime}\right) \widetilde{\varpi}_{\imath}(p) f_{\imath}(g)$ for $g \in G, p^{\prime} \leqq P$ - and $p \subseteq P$.

Proof. Let $1 \leqq i \leqq k, \alpha \in \Pi_{I}=\left\{\alpha_{k+1}, \cdots, \alpha_{l}\right\}, A \in \mathfrak{g}\left(\alpha^{\prime}\right)$ and $A^{\prime} \in \mathfrak{g}(\alpha)$. Then we have $A v_{\varpi_{i}}=0$ and $\alpha^{2} v_{\varpi_{i}}=0$. Hence by the representation theory of $s l_{2}$, $A^{\prime} v_{\varpi_{i}}=0$ and $f_{\imath}\left(g \exp A^{\prime}\right)=\left\langle v_{-\varpi_{i}}, g \exp A^{\prime} \cdot v_{\varpi_{i}}\right\rangle=\left\langle v_{-\omega_{i}}, g v_{\varpi_{i}}\right\rangle=f_{\imath}(g)$. Thus we get the relative invariance with respect to $P$. The relative invariance with respect to $P_{-}$can be proved in the same way.

9.11. Let us prove that $L_{4} \cong L_{7}$. It is enough to prove that

$$
p_{Y}^{*} H_{Y(w)}^{c d(w)}\left(\mathcal{O}_{Y}(\lambda)\right)=H_{B w P}^{c d(w)}\left(w \Omega^{\prime}(-\lambda)\right)
$$

(cf. (5.4)). Let $j_{G}: B_{-} \cdot P \rightarrow G$ and $j_{Y}: B_{-} \cdot y_{0} \rightarrow Y$ be inclusion mappings. Then $w^{-1} H_{B w P}^{c d(w)}\left(w \Re^{\prime}(-\lambda)\right)=\left(j_{G}\right)_{*} H_{w^{-1} B w P}^{c d(w)}\left(\Re^{\prime}(-\lambda) \mid B_{-} \cdot P\right)$. Since $w^{-1} H_{Y(w)}^{c d(w)}\left(\mathcal{O}_{Y}(\lambda)\right)=$ $\left(j_{Y}\right)_{*} H_{w^{-1}\left({ }^{\prime}(w)\right.}^{c d)}\left(\mathcal{O}\left(U_{-}, \lambda\right) \mid B_{-} \cdot y_{0}\right)$, it is enough to show that $p_{Y}^{*} \mathcal{O}\left(U_{-}, \lambda\right)=\Re^{\prime}(-\lambda)$ on $B_{-} \cdot P$. Let $\lambda=\sum_{\imath=1}^{k} \lambda_{\imath} \widetilde{\sigma}_{i}$ and $f^{-\lambda}=\prod_{i=1}^{k} f_{\imath}^{-\lambda_{\imath}}$. Then $f^{-\lambda}$ gives a multi-valued holomorphic function on $G \backslash \bigcup_{\imath=1}^{k} f_{\imath}^{-1}(0)=B_{-} \cdot P$. (Cf. (9.8) and (9.9).) Denote by $\mathcal{O} f^{-\lambda}$ the $\mathcal{O}$-module on $B_{-} \cdot P$ generated by $f^{-\lambda}$. Then $\mathcal{O} f^{-\lambda}=\mathscr{D} f^{-\lambda}=\mathfrak{I}^{\prime}(-\lambda) \mid B_{-} \cdot P$. By (9.10), $f^{-\lambda}$ gives a section of $F(\lambda, P)$ on $U_{-} \cdot P_{0}$, where $P_{0}$ is a connected, simply connected open neighbourhood of the identity element of $P$. (See (2.2) for $F(\lambda, P)$.) Thus $f^{-\lambda}$ determines a section of $\mathcal{O}^{a n}\left(U_{-}, \lambda\right)=\iota_{*}\left(p_{Y}^{a}{ }^{n} \mid U_{-} \cdot P_{0}\right)_{*}(F(\lambda$, $\left.P) \mid U_{-} \cdot P_{0}\right)$. Take a single-valued branch of $f^{-\lambda}$ on $U_{-} \cdot P_{0}$ so that $f^{-\lambda}(e)=1$. Then $f^{-\lambda} \equiv 1$ on $U_{-}$, i. e.. $f^{-\lambda}$ determines $1 \subseteq \Gamma\left(U_{-} \cdot y_{0}, \mathcal{O}_{Y}\right)=\Gamma\left(U_{-} \cdot y_{0}, \mathcal{O}\left(U_{-}, \lambda\right)\right)$, which we have denoted by $1^{\lambda}$ in (9.1). For $g \subseteq G$, we have $\left(L(g) f^{-\lambda}\right)(x)=$ $f^{-\lambda}\left(g^{-1} x\right)=\left(p_{Y}^{*} \varphi_{g}\right)(x) f^{-\lambda}(x)$ with a locally defined analytic function $\varphi_{g}$ on $Y$. 
For $A \in \mathrm{g}$, let $\varphi_{A}=\left.(d / d t) \varphi_{\exp t A}\right|_{t=0}$. Then $\varphi_{A}$ is a regular function, $A \cdot f^{-\lambda}=$ $\left(p_{Y}^{*} \varphi_{A}\right) \cdot f^{-\lambda}$, and $A \cdot 1^{\lambda}=\varphi_{A} \cdot 1^{\lambda}$. Hence $1 \otimes 1^{\lambda} \boxminus p_{Y}^{*} \mathscr{D}_{Y}(\lambda) \bigotimes_{p_{Y}^{-1} \mathscr{D}_{Y}(\lambda)} p_{Y}^{-1} \mathcal{O}\left(U_{-}, \lambda\right)=$ $p_{Y}^{*} \mathcal{O}\left(U_{-}, \lambda\right)$ satisfies $A\left(1 \otimes 1^{\lambda}\right)=\left(p_{Y}^{*} \varphi_{A}\right)\left(1 \otimes 1^{\lambda}\right)$. Thus all the linear differential equations satisfied by $f^{-\lambda}$ are also satisfied by $1 \otimes 1^{\lambda}$. Since $\mathcal{O}_{B_{-} \cdot P} f^{-\lambda}$ and $p_{Y}^{*}\left(\mathcal{O}_{B_{-} \cdot y_{0}} 1^{\lambda}\right)$ are integrable connections of rank one, $\Re^{\prime}(-\lambda) \mid B_{-} \cdot P=\mathcal{O}_{B_{-} \cdot P} f^{-\lambda}=$ $p_{Y}^{*}\left(\mathcal{O}_{B_{-} \cdot y_{0}} 1^{\lambda}\right)=p_{Y}^{*} \mathcal{O}\left(U_{-}, \lambda\right)$. Thus we have completed the proof of (9.4).

9.12. As an application of (9.4), we get a criterion for the simplicity of $V(w, \lambda, \mathfrak{p})$ in terms of $\mathscr{D}$-modules under certain assumptions. Here we restrict ourselves to the generalized Verma modules.

Let $\lambda=\lambda_{c}+\lambda_{d}$ be a character of $\mathrm{t}$, where $\lambda_{c}$ is $W_{I}$-invariant and $\lambda_{d}$ is a highest weight of a finite dimensional $P$-module. First, assume that

(9.12.1) $\Pi_{I}=\left\{\alpha_{k+1}, \cdots, \alpha_{l}\right\}$ with $k>0$, and

(9.12.2) $\left\langle\lambda+\rho, \alpha^{`}\right\rangle \neq 0,-1,-2, \cdots$ for any $\alpha \in R_{+}$.

Let $\boldsymbol{W}_{0}$ be the characteristic variety of $\eta^{\prime}\left(\lambda_{c}\right)\left[f_{1}^{-1}, \cdots, f_{k}^{-1}\right]$. Consider two more assumptions that

(9.12.3) for any $i \leqq k$, there exists $P_{\imath} \in \mathscr{D}_{G}$ and $b_{\imath}(\underline{s}) \equiv \boldsymbol{C}[\underline{s}]$ such that $P_{\imath} \underline{f}^{\underline{s}+c_{i}=}$ $b_{\imath}(\underline{s}) f^{s}$, where $f^{s}=f_{1}^{s_{1}} \cdots f_{k}^{s} k$ and $e_{\imath}=(0, \cdots, 0,1,0, \cdots, 0)$ (1 appears as the $i$-th component), and that

(9.12.4) there exists a point $p \in \boldsymbol{W}_{0}$ and for any $i \leqq k$, there exists an invertible micro-differential operator $Q_{\imath}$ in a neighbourhood of $p$ such that $Q_{i} \underline{s}^{\underline{s}+e_{i}}=b_{i}(\underline{s}) f^{\underline{s}}$ with the same $b_{i}(\underline{s})$ 's as in (9.12.3).

Let us identify $\underline{\lambda}_{c}=\left(\lambda_{1}, \cdots, \lambda_{k}\right) \subseteq C^{k}$ with $\lambda_{c}=\sum_{i=1}^{k} \lambda_{i} \varpi_{\imath}$.

Theorem 9.13. Under the above four assumptions, the following conditions are equivalent:

(1) The generalized Verma module $M(\lambda, \mathfrak{p}(I))$ is simple as a g-module.

(2) The generalized Verma module $M\left(-w_{S} \lambda_{,} \mathfrak{p}\left(w_{S} I w_{S}\right)\right)$ is simple as a g-module.

(3) The coherent $\mathscr{D}_{G}$-module $H_{B w_{S} P}^{0}\left(w_{S} \mathscr{I}^{\prime}\left(\lambda_{c}\right)\right)$ is simple, i.e., it does not have non-trivial coherent $\mathscr{D}_{G}$-submodules.

(4) $b_{i}\left(\lambda_{c}-\nu\right) \neq 0$ for any $1 \leqq i \leqq k$ and $\boldsymbol{\nu} \in \sum_{i=1}^{k} \boldsymbol{Z} \widetilde{\sigma}_{i}$.

Proof. Since the action of $-w_{s}$ on $t$ extends to an automorphism of $\mathfrak{g}$ preserving $\mathfrak{b}$, we get $(1) \Leftrightarrow(2)$. By (6.8) and (9.4), we get (2) $\Leftrightarrow(3)$. Since $w_{S}^{-1} B w_{S} P=G \backslash \bigcup_{i=1}^{k} f_{\imath}^{-1}(0)$ by (9.8) and (9.9), $w_{S}^{-1} H_{B w_{S} P}^{0}\left(w_{S} \eta^{\prime}\left(\lambda_{c}\right)\right)=\eta^{\prime}\left(\lambda_{c}\right)\left[f_{1}^{-1}, \cdots\right.$, $\left.f_{k}^{-1}\right]$. Hence we get $(3) \Leftrightarrow(4)$ by (8.4).

Remark 9.14. We assumed (9.12.1) only to exclude the trivial case. The author conjectures that the assumptions (9.12.3) and (9.12.4) are always satisfied. Thus the assumptions except (9.12.2) would be harmless. But (9.12.2) is essential and, because of this assumption, our irreducibility criterion is less 
complete than the one given by Jantzen [16]. In our forthcoming paper [13], we shall start to study the simplicity of generalized Verma modules and the $b$-functions of the semi-invariants without such assumptions. The relation between (9.13) and the result of Suga [31] will also become clear in [13].

\section{References}

[1] Beilinson, A. and Bernstein, J., Localisation de g-modules, C. R. Acad. Sci. Paris, 292 (1981), 15-18.

[2] Bernstein, I. N., Gelfand, I. M. and Gelfand, S. I., Differential operators on the base affine space and a study of g-modules, Lie groups and their representations, Proc. suminer school in group representations, (1975), 21-64, Halsted, New York.

[3] - Structure of representations generated by vectors of highest weight, Funct. Anal. Appl., 5 (1971), 1-8.

[4] Schubert cells and cohomology of the spaces G/P, Russian Math. Surveys, 28 (1973), 1-26.

[5] Bott, R., Homogeneous vector bundles, Ann. of Math., 66 (1957), 203-248.

[6] Bourbaki, N., Groupes et algèbres de Lie, chapitres 4, 5 et 6, Masson, 1981.

[7] - Algèbre commutative, chapitres 1 à 4, Masson, 1985.

[8] Brylinski, J.L. and Kashiwara, M., Kazhdan-Lusztig conjecture and holonomic systems, Invent. math., 64 (1981), 387-410.

[9] Dixmier, J., Algèbres enveloppantes, Gauthier-Villars, Paris, 1974.

[10] Gabber, O. and Joseph, A., On the Bernstein-Gelfand-Gelfand resolution and the Duflo sum formula, Compositio Math., 43 (1981), 107-131.

[11] Grothendieck, A. and Dieundonné, J. A., Eléments de géométrie algébrique, Springer, Berlin-Heidelberg-New York, 1971.

[12] Gyoja, A., Theory of prehomogeneous vector spaces without regularity condition, Publ. RIMS. Kyoto Univ., 27 (1991), 861-922.

[13] - Highest weight modules and $b$-functions of semi-invariants, preprint.

[14] Hecht, H., Miličić, D., Schmid, W. and Wolf, J. A., Localization and standard modules for real semisimple Lie groups I: The duality theorem, Invent. math., 90 (1987), 297-332.

[15] Hironaka, H., Resolution of singularities of an algebraic variety over a field of characteristic zero, Ann. of Math., 79 (1964), 109-326.

[16] Jantzen, J.C., Kontravariante Formen auf induzierten Darstellungen halbeinfacher Lie-Algebren, Math. Ann., 226 (1977), 53-65.

[17] Kashiwara, M., b-Functions and holonomic systems, Invent. math., 38 (1976), 33-53.

[18] - On the holonomic systems of linear differential equations, II, Invent. math., 49 (1978), 121-135.

[19] —-, Representation theory and $D$-modules on flag varieties, preprint.

$[20]$ - The universal Verma module and the $b$-function, Advanced studies in pure math., 6 (1985), 67-81.

[21] Kazhdan, D. and Lusztig, G., Representations of Coxeter groups and Hecke algebras, Invent. math., 53 (1979), 165-184.

[22] Kempf, G., The Grothendieck-Cousin complex of an induced representation, $A d v$. in Math., 29 (1978). 310-396.

[23] Laumon, G., Sur la catégorie dérivée des D-modules filtrés, Springer Lecture Note, 1016 (1983), 151-237.

[24] Lepowsky, J., Generalized Verma modules, the Cartan-Helgason theorem, and the 
Harish-Chandra homomorphism, J. Algebra, 49 (1977), 470-495.

[25] Muller, I., Rubenthaler, H. and Schiffmann, G., Structures des espaces préhomogènes associés à certaines algèbres de Lie graduées, Math. Ann., 274 (1986), 95-123.

[26] Quillen, D., Projective modules over polynomial rings, Invent. math., 36 (1976), $167-171$.

[27] Rubenthaler, H., Espaces préhomogènes de type parabolique, Lec. in Math., Kyoto Univ., 14 (1982), 189-221.

[28] Sabbah, C., Proximité évanescente II, Compositio Math., 64 (1987), 213-241.

[29] Sato, M., Kashiwara, M., Kimura, T. and Oshima, T., Micro-local analysis of prehomogeneous vector spaces, Invent. math., 62 (1980), 117-179.

[30] Sato, M. and Kimura, T., A classification of irreducible prehomogeneous vector spaces and their relative invariants, Nagoya Math. J., 65 (1977), 1-155.

[31] Suga, S., Highest weight modules associated with classical irreducible regular prehomogeneous vector space of commutative parabolic type, Osaka J. Math., 28 (1991), 323-346.

[32] Suslin, A., Projective modules over a polynomial ring are free, Soviet Math. Dokl., 17 (1976), 1160-1164.

[33] Verma, D. N., Structure of certain induced representations of complex semisimple Lie algebras, Bull. Amer. Math. Soc., 74 (1968), 160-166. 
